\title{
Management of Hepatocellular Carcinoma in Japan: JSH Consensus Statements and Recommendations 2021 Update
}

\author{
Masatoshi Kudo ${ }^{a}$ Yusuke Kawamura $^{\mathrm{b}}$ Kiyoshi Hasegawa ${ }^{c}$ Ryosuke Tateishid ${ }^{d}$ Kazuya Kariyama ${ }^{e}$ \\ Shuichiro Shiina ${ }^{f}$ Hidenori Toyodag $^{9}$ Yasuharu Imai $^{\text {h }}$ Atsushi Hiraoka $^{\mathrm{i}}$ Masafumi lkeda $^{\mathrm{j}}$ \\ Namiki Izumi $^{k}$ Michihisa Moriguchi ${ }^{l}$ Sadahisa Ogasawaram ${ }^{m}$ Yasunori Minami $^{a}$ \\ Kazuomi Ueshima ${ }^{a}$ Takamichi Murakamin $^{\mathrm{n}}$ Shiro Miyayama $^{\circ}$ Osamu Nakashima ${ }^{\mathrm{p}}$ \\ Hirohisa Yano ${ }^{q}$ Michiie Sakamoto ${ }^{r}$ Etsuro Hatano ${ }^{s}$ Mitsuo Shimada $^{t}$ Norihiro Kokudo $^{u}$ \\ Satoshi Mochidav Tetsuo Takeharaw HCC Expert Panel of the Japan Society of Hepatology \\ ${ }^{a}$ Department of Gastroenterology and Hepatology, Kindai University Faculty of Medicine, Osaka, Japan; ${ }^{b}$ Department \\ of Hepatology, Toranomon Hospital, Tokyo, Japan; ' Hepato-Biliary-Pancreatic Surgery Division, Department of \\ Surgery, Graduate School of Medicine, The University of Tokyo, Tokyo, Japan; d Department of Gastroenterology and \\ Hepatology, Graduate School of Medicine, The University of Tokyo, Tokyo, Japan; 'Department of Gastroenterology, \\ Okayama City Hospital, Okayama, Japan; fDepartment of Gastroenterology, Juntendo University, Tokyo, Japan; \\ 9Department of Gastroenterology and Hepatology, Ogaki Municipal Hospital, Gifu, Japan; 'Department of \\ Gastroenterology, Ikeda Municipal Hospital, Osaka, Japan; 'Gastroenterology Center, Ehime Prefectural Central \\ Hospital, Matsuyama, Japan; 'Department of Hepatobiliary and Pancreatic Oncology, National Cancer Center Hospital \\ East, Kashiwa, Japan; ' Department of Gastroenterology and Hepatology, Musashino Red Cross Hospital, Tokyo, Japan; \\ 'Department of Molecular Gastroenterology and Hepatology, Kyoto Prefectural University of Medicine, Kyoto, Japan; \\ mDepartment of Gastroenterology, Graduate School of Medicine, Chiba University, Chiba, Japan; nepartment of \\ Radiology, Kobe University Graduate School of Medicine, Hyogo, Japan; `Department of Diagnostic Radiology, \\ Fukui-ken Saiseikai Hospital, Fukui, Japan; PDepartment of Clinical Laboratory Medicine, Kurume University Hospital, \\ Kurume, Japan; 'Department of Pathology, Kurume University School of Medicine, Kurume, Japan; 'Department \\ of Pathology, Keio University School of Medicine, Tokyo, Japan; 'Department of Gastroenterological Surgery, \\ Hyogo College of Medicine, Nishinomiya, Japan; 'Department of Surgery, Tokushima University, Tokushima, \\ Japan; "Department of Surgery, National Center for Global Health and Medicine, Tokyo, Japan; 'Department of \\ Gastroenterology and Hepatology, Saitama Medical University, Saitama, Japan; "Department of Gastroenterology and \\ Hepatology, Osaka University Graduate School of Medicine, Osaka, Japan
}

\section{Keywords}

Hepatocellular carcinoma $\cdot$ Consensus statement $\cdot$ Japan Society of Hepatology · Pathology · Diagnosis · Treatment

\section{Abstract \\ The Clinical Practice Manual for Hepatocellular Carcinoma was published based on evidence confirmed by the Evi- dence-based Clinical Practice Guidelines for Hepatocellular Carcinoma along with consensus opinion among a Japan So-}

ciety of Hepatology (JSH) expert panel on hepatocellular carcinoma (HCC). Since the JSH Clinical Practice Guidelines are based on original articles with extremely high levels of evidence, expert opinions on HCC management in clinical practice or consensus on newly developed treatments are not included. However, the practice manual incorporates the literature based on clinical data, expert opinion, and real-world clinical practice currently conducted in Japan to facilitate its use by clinicians. Alongside each revision of the JSH Guidelines, we issued an update to the manual, with the first edi-
C 2021 The Author(s)

Published by S. Karger AG, Basel

This is an Open Access article licensed under the Creative Commons Attribution-NonCommercial-4.0 International License (CC BY-NC) (http://www.karger.com/Services/OpenAccessLicense), applicable to the online version of the article only. Usage and distribution for commercial purposes requires written permission.
Correspondence to:

Masatoshi Kudo, m-kudo@med.kindai.ac.jp 
tion of the manual published in 2007, the second edition in 2010, the third edition in 2015, and the fourth edition in 2020, which includes the 2017 edition of the JSH Guideline. This article is an excerpt from the fourth edition of the HCC Clinical Practice Manual focusing on pathology, diagnosis, and treatment of HCC. It is designed as a practical manual different from the latest version of the JSH Clinical Practice Guidelines. This practice manual was written by an expert panel from the JSH, with emphasis on the consensus statements and recommendations for the management of HCC proposed by the JSH expert panel. In this article, we included newly developed clinical practices that are relatively common among Japanese experts in this field, although all of their statements are not associated with a high level of evidence, but these practices are likely to be incorporated into guidelines in the future. To write this article, coauthors from different institutions drafted the content and then critically reviewed each other's work. The revised content was then critically reviewed by the Board of Directors and the Planning and Public Relations Committee of JSH before publication to confirm the consensus statements and recommendations. The consensus statements and recommendations presented in this report represent measures actually being conducted at the highest-level HCC treatment centers in Japan. We hope this article provides insight into the actual situation of HCC practice in Japan, thereby affecting the global practice pattern in the management of HCC.

2021 The Author(s)

Published by S. Karger AG, Basel

\section{Introduction}

The first edition of the Clinical Practice Guidelines for Hepatocellular Carcinoma was published in 2005 [1-3], the second edition in $2009[4,5]$, the third edition in 2013 $[6,7]$, and the fourth edition in $2017[8,9]$ by the Japan Society of Hepatology (JSH). The Clinical Practice Manual for Hepatocellular Carcinoma was published based on evidence confirmed by the Evidence-based Clinical Practice Guidelines for Hepatocellular Carcinoma along with consensus opinion among a JSH expert panel on hepatocellular carcinoma (HCC), although the level of evidence is not as high as that of the guidelines. The manual incorporates the literature based on clinical data, expert opinion, and real-world clinical practice in Japan to facilitate its use by clinicians. Alongside each revision of the JSH Guidelines, we issued an update to the manual, with the first edition of the manual published in 2007 [10, 11], the second edition in 2010 [12, 13], the third edition in 2015 [14-16], and the fourth edition in 2020 [17], which in- cludes the 2017 edition of the JSH Guideline. This article is an excerpt from the fourth edition of the manual [17] focusing on pathology, diagnosis, and treatment of HCC. It is designed as a practical manual based on the latest version of the JSH Clinical Practice Guidelines $[8,9]$. This manual was written by an expert panel from the JSH (see online suppl. Table 1; for all online suppl. material, see www.karger.com/doi/10.1159/000514174), with emphasis on the consensus statements and recommendations for the management of HCC proposed by the JSH expert panel.

\section{Pathology of HCC: Important Issues for Clinicians}

\section{Consensus Statements}

1. The small nodular type with indistinct margins is currently considered clinically diagnosable, earlystage HCC and is termed "early HCC."

2. Early HCC rarely shows hypervascularity on angiography or contrast-enhanced computed tomography (CT).

3. Fatty change is considered a typical morphological feature of early HCC.

4. Locoregional treatment (ablation or transarterial chemoembolization [TACE]) for HCCs of the simple nodular type with extranodular growth and HCCs of the confluent multinodular type requires special attention because these types are associated with increased risk of intrahepatic metastasis and recurrence compared with the small nodular type with indistinct margins and the simple nodular type.

5. The appearance of a "nodule-in-nodule" feature in well-differentiated HCC (early HCC) is an indication of increasing malignancy grade.

Understanding the pathology of the processes involved in tumor growth and development is important for the diagnosis and treatment of HCC. Therefore, the concept of early HCC and an understanding of multistep hepatocarcinogenesis is essential for physicians treating patients with HCC. The degree of progression of HCC cannot be determined according to the depth of invasion because the liver does not have a layered structure like the gastrointestinal tract. Moreover, defining early HCC is difficult because of the relatively high frequency of synchronous or metachronous multicentric incidence. However, emerging evidence indicates that the pathological appearance and biological malignancy of HCC change with increasing tumor size, and accordingly, the features of liver lesions equivalent 


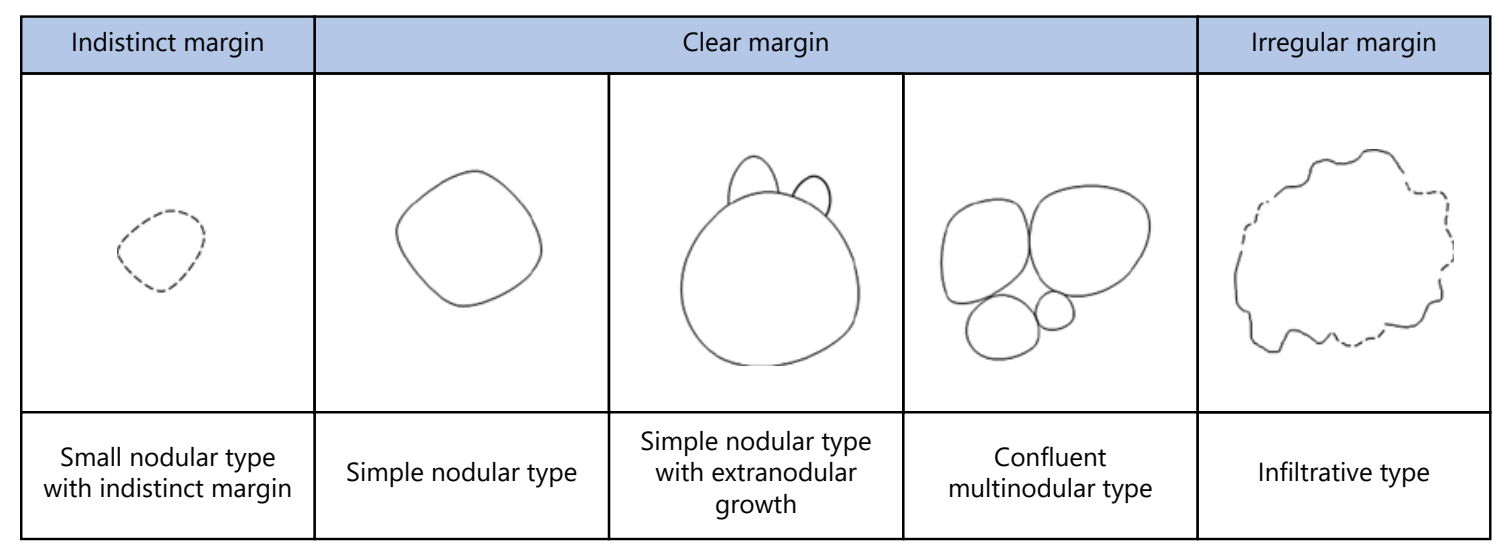

Cited from ref.20 with permission

Fig. 1. Schematic representation of gross pathological type of HCC. HCC, hepatocellular carcinoma. Reproduced with permission from the Liver Cancer Study Group of Japan [20].

to early-stage cancers in other organs are becoming increasingly clearer $[18,19]$.

\section{Definition of Early HCC by Pathological Appearance}

The General Rules for the Clinical and Pathological Study of Primary Liver Cancer, Revised Sixth Edition [20], defines 5 types of HCC based on macroscopic features: small nodular type with indistinct margin, simple nodular type, simple nodular type with extranodular growth, confluent multinodular type, and infiltrative type (Fig. 1). Because cases classified as infiltrative type are rare, this section addresses the remaining 4 types. In addition, small-sized HCC can be macroscopically divided into the well-defined simple nodular type and the poorly defined indistinct margin type. Histologically, most simple nodular-type lesions are encapsulated moderately differentiated HCCs, whereas lesions of the indistinct margin type are well-differentiated HCCs with low-grade atypia. These HCCs are characterized by a smaller cell size with an increased nuclear-cytoplasmic ratio, increased cell density, 2-3 layers of thin cord-like structures, and small pseudoglandular structures, which typically encompass the preexisting portal tract areas. At the growing tip of these tumors, cancer cells proliferate to replace hepatic cords in the non-cancerous area, resulting in a grossly indistinct and unidentifiable boundary. The small nodular type with indistinct margin is currently considered a clinically diagnosable minimal HCC and is termed "early HCC." Early HCC is associated with portal tract area infiltration (stromal invasion) and the absence of portal venous invasion or intrahepatic metastasis. Al- though differentiating between early HCC and highgrade dysplastic nodules is often difficult, it can be done based on the presence or absence of atypical cell infiltration into the portal tract area (stromal invasion) [21,22].

\section{Vascular Architecture of Early HCC}

Overt HCC is completely supplied by arteries, whereas early HCC receives both portal and arterial supply because it encompasses varying numbers of preexisting portal tract areas. However, because there are few portal tract areas in cancerous tissue (approximately 25\% of those in non-cancerous tissue) and the arterial tumor vessels are immature, the portal and arterial supply is insufficient, and the tumor area appears less vascularized than the non-tumor liver parenchyma. Although the arterial tumor vessels develop in parallel with tumor growth, even tumors approximately $10 \mathrm{~mm}$ in diameter are undervascularized, and capillarization or neovascularization of the sinusoidal blood spaces in the tumor stroma is inadequate. For these reasons, early HCC often shows no hypervascularity on angiography or contrast-enhanced CT (CECT).

\section{Fatty Changes in Early HCC}

Small, early HCCs are often visualized as hyperechoic nodules on abdominal ultrasonography, and many tumors contain cancerous cells that have undergone fatty change. Fatty change in HCC occurs most frequently in tumors measuring 10-15 $\mathrm{mm}$ in diameter (approximately $40 \%$ ), and it becomes less common as tumor size increases and the degree of differentiation decreases. Thus, 
Table 1. Gross pathological type and histopathologic findings

\begin{tabular}{lcccccc}
\hline & fc & fc-inf & sf & Vp & Vv & im \\
\hline Small nodular type with indistinct margin, $n(\%)$ & 0 & 0 & $2(9.1)$ & 0 & 0 & 0 \\
Simple nodular type, $n$ (\%) & $90(73.2)$ & $79(64.2)$ & $65(52.8)$ & $23(18.7)$ & $3(2.4)$ & $5(4.1)$ \\
Simple nodular type with extranodular growth, $n(\%)$ & $38(84.4)$ & $35(77.8)$ & $35(77.8)$ & $20(44.4)$ & $2(4.4)$ & $12(26.7)$ \\
Confluent multinodular type, $n(\%)$ & $1(5.3)$ & $1(5.3)$ & $14(73.7)$ & $12(63.2)$ & $3(15.8)$ & $5(26.3)$ \\
\hline
\end{tabular}

fc, capsule formation; fc-inf, capsule invasion; sf, septum formation; Vp, portal vein invasion; Vv, hepatic vein invasion; im, intrahepatic metastasis.

Table 2. Pathological differentiation and mean tumor size according to gross pathological type of $\mathrm{HCC}$ (resected $\mathrm{HCC} \leq 3 \mathrm{~cm}$ )

\begin{tabular}{llcccr}
\hline & $\begin{array}{l}\text { Well, } \\
n(\%)\end{array}$ & $\begin{array}{l}\text { Well + mod, } \\
n(\%)\end{array}$ & $\begin{array}{l}\text { Mod, } \\
n(\%)\end{array}$ & $\begin{array}{l}\text { Mod + poor, } \\
n(\%)\end{array}$ & $\begin{array}{c}\text { Total } \\
\text { sumor mm }\end{array}$ \\
\hline Small nodular type with indistinct margin & $19(86.4)$ & $3(13.6)$ & 0 & 0 & 22 \\
Simple nodular type & $6(4.9)$ & $24(19.5)$ & $92(74.8)$ & $1(0.8)$ & $13.6 \pm 5.4$ \\
Simple nodular type with extranodular growth & 0 & $5(11.1)$ & $40(88.9)$ & 0 & $22.8 \pm 5.6$ \\
Confluent multinodular type & 0 & $6(31.6)$ & $11(57.9)$ & $2(10.5)$ & $23.1 \pm 5.4$ \\
\hline
\end{tabular}
HCC.

HCC, hepatocellular carcinoma; Well, well-differentiated HCC; Mod, moderately differentiated HCC; poor, poorly differentiated

fatty change is considered a morphological feature of early HCC. This fatty change is attributed to temporary ischemia of the cancerous nodules; this is caused by a decrease in the portal and arterial blood supply related to the few portal tract areas and immature arterial tumor vessels (unpaired arteries) in tumors measuring $10-15 \mathrm{~mm}$ [23].

Diagnostic Imaging of Early HCC: Usefulness of GdEOB-DTPA-Enhanced Magnetic Resonance Imaging

Early HCCs are typically hypovascular and are thus unlikely to be detected using CECT, which visualizes arterial blood flow. This limits the confirmation rate when using CECT. Gd-EOB-DTPA contrast-enhanced magnetic resonance imaging (EOB-MRI) assesses hepatocyte function and can identify HCC as a mass with decreased contrast-uptake in the hepatobiliary phase. A group from the Kurume University reviewed imaging data from approximately 50 cases (both CECT and EOB-MRI were performed in each case) diagnosed as well-differentiated (early) HCC on biopsy. The results showed that the diagnostic rate for HCC was around 60\% with CECT based on decreased portal blood flow (including CT during hepatic arteriography [CTHA] and CT during arterioportography [CTAP]) compared with approximately $80 \%$ when using EOB-MRI, suggesting that the diagnostic ac- curacy was superior with EOB-MRI. However, there are unresolved issues associated with EOB-MRI, including the presence of contrast-uptake in some overt moderately differentiated HCCs [24] and non-contrast-uptake benign nodules, suggesting the need for further research.

\section{Macroscopic Classification and Malignancy Grade of HCCs}

The association between macroscopic type and malignancy grade is related to the degree of histological differentiation. An analysis of resected HCC lesions measuring $\leq 3 \mathrm{~cm}$ in diameter showed that approximately $85 \%$ of the indistinct margin type (early HCC) consists of homogeneous, well-differentiated HCC tissue, whereas the remaining 15\% encompasses an area consisting of de-differentiated, moderately differentiated HCC tissue, with clear or obscure "nodule-in-nodule" appearance.

HCCs of the indistinct margin type are not associated with intrahepatic metastasis and PVI, and they are significantly smaller than other nodular-type HCCs, with a mean tumor diameter of approximately $13.6 \mathrm{~mm}$. Approximately $75 \%$ of simple nodular-type HCCs are moderately differentiated HCCs, with histological evidence of portal vein invasion in $20 \%$ of cases and intrahepatic metastasis in $4 \%$ of cases, which are considered classical 
Table 3. Distance between main HCC nodule and intrahepatic metastatic nodule according to gross pathological type

\begin{tabular}{|c|c|c|c|c|c|}
\hline & \multicolumn{5}{|c|}{ Distance, $\mathrm{mm}$} \\
\hline & 2 & $2.1-5$ & $5.1-10.0$ & $>10.1$ & total \\
\hline Simple nodular type, $n(\%)$ & $6(66.7)$ & $1(11.1)$ & $0(0.0)$ & $2(22.2)$ & 9 \\
\hline Simple nodular type with extranodular growth, $n(\%)$ & $23(30.7)$ & $12(16.0)$ & $17(22.7)$ & $23(30.7)$ & 75 \\
\hline Confluent multinodular type, $n(\%)$ & $27(41.5)$ & $19(29.2)$ & $13(20.2)$ & $6(9.2)$ & 65 \\
\hline Total, $n(\%)$ & $56(37.6)$ & $32(21.5)$ & $30(20.1)$ & $31(20.8)$ & 149 \\
\hline
\end{tabular}

overt HCC. Approximately 49-79\% of HCCs of the simple nodular type with extranodular growth and the confluent multinodular type correspond to more advanced HCCs. Most of these are composed of moderately to poorly differentiated HCC tissue and are more frequently associated with PVI and intrahepatic metastasis than the simple nodular type (Tables 1,2$)$. These lesions are also associated with more intrahepatic metastases and longer distance (further away) from the main tumor than the simple nodular type [25] (Table 3). These observations indicate that HCCs of higher biological malignancy grade exhibit macroscopic features of the simple nodular type with extranodular growth and the confluent multinodular type. Thus, for HCCs of the simple nodular type with extranodular growth and for those of the confluent multinodular type, locoregional therapy such as ablation or TACE should be avoided because these HCC types are associated with a higher risk of intrahepatic metastasis and recurrence than those of the indistinct margin and simple nodular types.

Differentiation Level and Malignancy Grade of HCCs

Most HCCs are initially well differentiated and macroscopically appear as ill-defined, small nodular lesions; however, they increase in diameter as they undergo dedifferentiation. Moderately to poorly differentiated carcinoma tissue within the well-differentiated carcinoma tissue grows expansively because its proliferative capacity is greater than that of the surrounding well-differentiated HCC tissue; it eventually completely replaces the well-differentiated tissue to become classic HCC appearing as a distinct nodule $[20,26]$. In small HCCs with "nodule-innodule" appearance, carcinoma tissue in the differentiated internal area shows p53 overexpression at a rate of approximately $40 \%$ and a significantly higher labeling index for Ki-67, a marker for proliferative potential, than the surrounding well-differentiated carcinoma tissue. These observations indicate that the appearance of an intranodal nodule in well-differentiated carcinoma (early HCC) is an indication of increased malignancy. This is also consistent with the finding that the appearance of an intranodular nodule is associated with accelerated tumor growth, as detected on ultrasound at follow-up. These findings suggest that HCCs with "nodule-in-nodule" appearance are in the process of de-differentiation to progressed HCC and require treatment for HCCs. Cancerous nodules with "nodule-in-nodule" appearance are characterized by a marked difference in the degree of vascularity between the marginal well-differentiated HCC tissue and the internal moderately to poorly differentiated HCC tissue. On contrast-enhanced ultrasonography (CEUS) or CECT, the peripheral well-differentiated carcinoma tissue is visualized as a hypovascular area because of immature arterial tumor vessels and sinusoidal capillarization, whereas the internal moderately to poorly differentiated HCC tissue is visualized as a hypervascular region, as arterial tumor vessels are well developed. These observations suggest that the degree of arterial vascularity of HCC is also closely correlated with the degree of differentiation; therefore, the malignancy grade of early HCC can be determined to some extent based on angiographic findings.

HCC with sarcomatous change (sarcomatoid HCC) can develop rapidly and extensively. It is the least differentiated and most malignant form of HCC, and it is commonly referred to as sarcomatoid HCC. Sarcomatoid HCC can occur suddenly during the normal course of HCC or after TACE; however, it is also present in approximately $2 \%$ of patients who undergo relatively small surgical resection [27].

\section{HCC Size and Malignancy Grade}

The size (tumor diameter) of HCC is associated with gross morphology, histological degree of differentiation, frequency of intrahepatic metastasis, frequency of portal 
Table 4. Frequency of each gross pathological type according to tumor size (resected $1,409 \mathrm{HCCs} \leq 5 \mathrm{~cm}$ )

\begin{tabular}{lllllrrr}
\hline Gross pathological type & \multicolumn{2}{l}{ Tumor size, $\mathrm{mm}$} & & & & \\
\cline { 2 - 7 } & $\sim 10$ & $11-15$ & $16-20$ & $21-30$ & $31-40$ & $41-50$ & table \\
\hline Small nodular type with indistinct margin, $n(\%)$ & $51(48.6)$ & $44(25.4)$ & $24(8.6)$ & $24(5.5)$ & $3(1.2)$ & $0(0)$ & $146(10.4)$ \\
Simple nodular type, $n(\%)$ & $41(39.0)$ & $75(43.4)$ & $162(58.1)$ & $236(53.6)$ & $128(49.7)$ & $80(51.6)$ & $722(51.2)$ \\
Simple nodular type with extranodular growth, $n(\%)$ & $8(7.6)$ & $32(20.2)$ & $58(20.8)$ & $118(26.8)$ & $96(37.4)$ & $55(35.5)$ & $367(26.0)$ \\
Confluent multinodular type, $n(\%)$ & $5(4.8)$ & $22(12.7)$ & $35(12.5)$ & $62(14.1)$ & $30(11.7)$ & $20(12.9)$ & $174(12.4)$ \\
\hline Total & 105 & 173 & 279 & 440 & 257 & 155 & 1,409 \\
\hline
\end{tabular}

HCC, hepatocellular carcinoma.

Table 5. Rate of portal vein invasion and intrahepatic metastasis according to tumor size

\begin{tabular}{llrlll}
\hline \multicolumn{7}{c}{ Tumor size, cm } \\
\cline { 2 - 6 } & $0-1$ & $1.1-2.0$ & $2.1-3.0$ & $3.1-5.0$ & $5.1-10.0$ \\
\hline Vp, $n(\%)$ & 0 & 28.3 & 33.3 & 49.0 & 58.5 \\
$\mathrm{IM}, n(\%)$ & 0 & 6.7 & 17.1 & 29.6 & 43.9 \\
\hline
\end{tabular}

Vp, portal vein invasion; IM, intrahepatic metastasis.

vein invasion, and other parameters. HCCs of the indistinct margin type mostly measure $\leq 2 \mathrm{~cm}$ and are rarely $\geq 3 \mathrm{~cm}$. In addition, the frequency of the simple nodular type with extranodular growth and that of the confluent multinodular type increase with increasing tumor size (Table 4). Histologically, the frequency of homogeneous, well-differentiated HCC tissue decreases dramatically in relation to tumor size in tumors $>2 \mathrm{~cm}$ in diameter. These larger lesions are composed largely of moderately to poorly differentiated HCC tissue, and the frequency of portal vein invasion and intrahepatic metastasis also increases in correlation with tumor size (Table 5). Tumor size is positively correlated with malignancy grade, except in very slowly growing, large, well-differentiated HCCs [28], some of which are found in the non-cirrhotic liver and are often difficult to differentiate from hepatocellular adenoma (HCA).

HCC can proliferate and become more malignant as it de-differentiates from highly differentiated to moderately or poorly differentiated types; however, HCC exists in a wide variety of types, including early HCCs; large, welldifferentiated HCCs resembling HCA; HCCs with severe lymphocytic infiltration; and other low-grade types such as fibrolamellar carcinoma (FLC). In the context of the increasing prevalence of non-B non-C HCC, differentiating HCC from HCA can be challenging, both clinically and pathologically, as mentioned above. These challenges occur when well-differentiated HCC, FLC, and progressed HCC depicted as hyperintensity on hepatobiliary phase of EOB-MRI need to be differentiated from HCA by imaging and/or liver biopsy.

There are several subtypes of HCA [29], including those with pathological features similar to those of welldifferentiated HCC or FLC, those at high risk of carcinogenesis, and those prone to rupture and bleeding. Therefore, when differential diagnosis between HCA and HCC is necessary based on the patient's age and general condition, immunohistochemistry is recommended to establish a pathological diagnosis.

\section{Diagnosis of HCC}

\section{Tumor Markers}

\section{Consensus Statements}

6. The tumor markers alpha-fetoprotein (AFP), Lens culinaris-agglutinin-reactive fraction of AFP (AFPL3), and protein induced by vitamin K absence-II/ des-gamma-carboxy prothrombin (PIVKA-II/ DCP) are often elevated in HCC, and the positivity rate of these markers increases with disease progression.

7. In patients with elevated tumor marker levels, failure to detect a lesion using 1 imaging modality should be checked using a different imaging modality.

8. For all 3 tumor markers, setting higher cutoff values results in decreased sensitivity and increased specificity, whereas setting lower cutoff values increases 
sensitivity but decreases specificity. Appropriate cutoff values should be selected based on the intended purpose, such as HCC surveillance, diagnosis, evaluation of treatment response, and follow-up for recurrence.

9. These markers can be easily measured repeatedly, and following the time course of these markers (i.e., change over time) is useful for detecting and diagnosing HCC.

10. Other factors affect tumor marker levels, and values may be elevated even in the absence of HCC.

11. The appropriate combination of 3 tumor markers may enhance their diagnostic performance.

There are 3 major tumor markers for HCC: AFP, AFPL3 fraction, and PIVKA-II/DCP [30-33]. The expected diagnostic value of tumor markers includes (1) determining the presence/absence of disease (early diagnosis, determining the degree of cancer progression), (2) qualitative diagnosis (differential diagnosis, determining the degree of malignancy), and (3) evaluating treatment response and detecting recurrence. Since no single tumor marker can satisfy all criteria, combining the above 3 markers can increase their diagnostic performance. The recommended cutoff values of AFP, AFP-L3 fraction, and PIVKA-II/DCP for HCC surveillance and diagnosis are $10 \mathrm{ng} / \mathrm{mL}, 10 \%$, and $40 \mathrm{mAU} / \mathrm{mL}$, respectively.

\section{Imaging Diagnosis}

Consensus Statements

12. Ultrasonography is the first-line imaging modality for screening patients at high risk of HCC, and CEUS can assess hemodynamics and Kupffer cell function.

13. CECT plays a major role in the diagnostic imaging of HCC because of its capacity for assessing arterial vascularity in lesions through dynamic studies, as well as its widespread availability.

14. Contrast-enhanced MRI, particularly dynamic studies with EOB-MRI, can be used for assessing hemodynamics and hepatocyte function in the hepatobiliary phase and is thus useful for detecting and differentiating between malignant and benign lesions.

15. CTHA and CTAP provide the most accurate assessment of hemodynamic changes in hepatocellular nodules during multistep carcinogenesis; however, its invasive technical nature limits applicability in the routine clinical setting.

Diagnostic imaging is used for determining the presence or absence of disease and for making a differential diagnosis during screening and mainly includes ultrasound, CT, and MRI. For surveillance of groups at high

JSH Consensus Statements and Recommendations on HCC risk for HCC, regular ultrasound follow-up is recommended because of its simplicity and low cost. For the differential diagnosis of ultrasound-identified lesions, dynamic studies are recommended in which CT/MRI images are acquired over time after bolus intravenous infusion of contrast agent. Dynamic studies to visualize the hemodynamic and histopathological characteristics of HCC are essential to differentiate typical hypervascular HCC from other hypervascular benign lesions or falsepositive lesions to confirm the diagnosis. MRI has the advantages of high tissue contrast and the capacity to visualize normal tissue within a lesion even without use of contrast agent. Contrast-enhanced MRI using the liver-specific contrast agent Gd-EOB-DTPA is most commonly performed in Japan, and the use of extracellular fluid or reticuloendothelial system (RES) contrast agents is limited. CTHA and CTAP provide a highly sensitive assessment of blood flow; however, the invasive nature of the procedure limits its application to the differential diagnosis of liver lesions.

\section{Ultrasonography}

Ultrasonography is used as a screening test for patients at high risk of HCC because of hepatitis B/C virus-associated chronic hepatitis and cirrhosis and for those at very high risk with prior history of treatment for HCC. Ultrasonography has the advantages of being minimally invasive and easily repeatable. CEUS, which can be used for assessing hemodynamics and Kupffer cell function, has a high diagnostic performance. However, this modality is not fully effective for examining the area under the dome of the diaphragm because of a blind area or in patients with severe obesity or hepatic atrophy. Ultrasound tests used in HCC screening and diagnosis include B-mode, Doppler, and CEUS.

\section{B-Mode Ultrasound}

Common findings in HCC include a "mosaic pattern" (differing levels of echogenicity in different areas divided by fibrous septa) and a "halo" (a thin marginal hypoechoic zone caused by a pseudocapsule). Well-differentiated early HCCs $(<1.5 \mathrm{~cm})$ are less likely to show these typical findings and are visualized as hyperechoic or hypoechoic areas with indistinct boundaries within the background liver; however, detecting these lesions is often difficult and requires examiners with a certain level of technical skill.

\section{Color Doppler Ultrasound}

Hypervascular HCCs can be identified on color Doppler ultrasound by a "basket pattern," which represents 
pulsatile arterial blood flow in tumor vessels from the margins to the center of the tumor [34]. In pathologically early HCC, blood flow inside the tumor is not pulsatile flow, suggesting intra-tumoral portal flow, and neovascularization is usually unremarkable.

\section{CEUS with Sonazoid ${ }^{\circledR}$}

\section{Consensus Statements}

16. CEUS is the most sensitive tool to detect intra-tumoral arterial vascularity. CEUS can simultaneously assess the liver tumor hemodynamics, vascular architecture, Kupffer cell function, and gross tumor morphology and is thus useful for the accurate diagnosis of HCC and for determining the malignancy grade.

17. The clinical indications for CEUS in HCC include detection, differential diagnosis, diagnosis of malignancy grade, puncture needle guidance for percutaneous treatment, and evaluating treatment response.

18. CEUS should be used to complement CECT/MRI because of the presence of a diagnostic blind area with CEUS, such as the liver segment under the dome of the diaphragm and because of its varying diagnostic accuracy, which depends on patient factors such as obesity, liver atrophy, and lesion depth.

In CEUS with contrast agent Sonazoid, a diagnosis is made by assessing enhancement patterns in 2 phases: the vascular phase lasting up to $3 \mathrm{~min}$ after intravenous infusion and the post-vascular phase (Kupffer phase) starting after 10 min $[35,36]$. In hypervascular HCCs, the entire tumor is intensely enhanced in the arterial phase, whereas the enhancement is washed out in the portal phase. The lower the degree of differentiation, the more rapid the washout. Intrahepatic cholangiocarcinoma (ICC) can also be correctly differentiated from HCC because of rapid washout during vascular phase and complete defect on the Kupffer phase. The signals in the Kupffer phase are emitted from microbubbles of the contrast agent phagocytosed by Kupffer cells that resonates on ultrasound [36, 37]. Therefore, hypervascular typical HCCs, which lack Kupffer cells, show decreased or no enhancement, whereas well-differentiated HCCs, which contain Kupffer cells, appear isoechoic or slightly hypoechoic with the background liver. In defect reperfusion imaging, Sonazoid is reinjected in the Kupffer phase and is effective for detecting new lesions during surveillance $[38,39]$ and for guiding ablation therapy $[40,41]$. This technique is called "defect reperfusion imaging (DRPI)" or reinjection method [41]. CEUS is inferior to CT/MRI in terms of objectivity, although it is highly advantageous because it can be used regardless of renal dysfunction or iodine allergy.
CEUS is characterized by its capacity for simultaneously assessing tumor blood flow and Kupffer cell function, and its diagnostic accuracy is improving yearly as advances are made in image processing technology. The indications for CEUS in HCC practice include (1) identifying the presence of a nodule poorly visualized by Bmode ultrasound because of a coarse liver parenchyma associated with cirrhotic liver; (2) differential diagnosis of liver tumors, including borderline lesions or dysplastic nodules; (3) evaluating HCC malignancy grade; (4) needle insertion guidance during percutaneous treatment; and (5) evaluating treatment response after TACE or ablation.

\section{Differential Diagnosis of Liver Tumors by CEUS}

CEUS is characterized by high temporal and spatial resolution and a capacity for assessing multiple parameters simultaneously, such as liver tumor hemodynamics, vascular architecture, presence or absence of Kupffer cells, and tumor morphology $[42,43]$. This modality is thus more useful for differential diagnosis than other modalities. The diagnostic algorithm of the JSH Clinical Practice Guidelines for HCC 2017 suggests that lesions with atypical findings on CECT/MRI are a good indication for CEUS. Typical CEUS findings enable differentiation between types of liver tumors including HCC and ICC [44].

\section{CEUS-Based Malignancy Grading of HCC}

The gross classification and histological differentiation level of HCC indicate its biological malignancy, and CEUS is used as a precision diagnostic tool to determine these characteristics. Regarding the gross classification of HCCs, the simple nodular type with extranodular growth and the confluent multinodular type are generally of higher malignancy grade than the simple nodular type [25]. CEUS Kupffer imaging is useful for assessing the tumor contours, provides superior diagnostic accuracy over CECT, and is complementary to EOB-MRI $[45,46]$. CEUS tumor vascular and Kupffer images reflect the degree of histological differentiation; as the degree of histological differentiation decreases, the degree of vascular irregularity on vascular phase images increases from "fine" to "vascular" and to "irregular," and the echogenicity ratio of carcinoma to non-carcinoma areas in the Kupffer phase decreases [47, 48]. Particular attention should be paid to nodules showing irregularity on vascular images, as these lesions are significantly more likely to be poorly differentiated HCCs and are associated with a high risk of portal vein invasion. 


\section{CEUS for Treatment Guidance}

As mentioned above, CEUS Kupffer phase imaging is useful for diagnosing the presence of nodules that are poorly visualized on B-mode ultrasound. This technique can be further combined with DRPI to reliably identify posttreatment residual and recurrent lesions, which makes it a useful tool for treatment guidance [41]. In the actual procedure of CEUS-guided puncture of a nodule poorly visualized on B-mode ultrasound, the lesion is identified using DRPI and punctured under Kupffer phase imaging, which allows long-term, stable visualization of the target tumor. For nodules that cannot be identified even by CEUS, contrast-enhanced fusion imaging with CT/MRI images is useful [49].

\section{Computed Tomography}

Overt HCC usually present hypervascularity on arterial phase and washout on portal venous phase $[50,51]$. However, overt HCC can sometimes present with atypical findings [50]. On plain CT, these lesions may be isoattenuation and thus undetectable. Lesions with marked fat deposition can be visualized as clear hypoattenuation areas on plain CT; however, they may be poorly enhanced by the contrast agent in the early phase of contrast enhancement. Some lesions show hyperenhancement in the early phase, suggesting hypervascular lesions but do not show "washout" in the late phase. These lesions, which are visualized as small enhanced lesions only in the early phase, are difficult to differentiate from arterial-portal (A-P) shunts, false-positive lesions due to abnormal venous reflux, hepatic cavernous hemangiomas, or focal nodular hyperplasia. Similarly, isoattenuation or hypoattenuation lesions in the arterial phase can be challenging to differentiate from regenerative or dysplastic nodules and early HCC. Poorly differentiated HCCs can also show hypovascular features. MRI, with its high tissue contrast and a wide variety of available contrast agents, is effective for differentiating between these HCCs presenting with atypical findings and false-positive lesions.

\section{Magnetic Resonance Imaging}

MRI has the advantages of high tissue contrast and the ability to visualize tissue properties within a lesion even without use of a contrast agent. On T1-weighted images, overt HCCs show variable signal intensity and can be visualized as hypo-, iso-, or hyperintense lesions, whereas early HCCs and dysplastic nodules are generally hyperintense. T2-weighted images are useful for differentiating overt HCCs, which are detected as hyperintense lesions, from early HCCs, which are depicted as iso- to hypoin- tense lesions [52]. Comparison of in-phase, out-of-phase, and fat-suppressed T1-weighted images is useful for assessing the fat component of a lesion. Diffusion-weighted images are also actively used to identify lesions and predict the degree of differentiation [53]. There are 3 major types of contrast agents for MRI: extracellular fluid, RES, and hepatocyte-specific contrast agents. In Japan, the most common MRI technique is EOB-MRI with hepatocyte-specific contrast agents. Recent improvements in the performance of MRI scanners and the development of fast imaging using T1-weighted images with 3D Fourier transformation have enabled multiphase dynamic MRI of the whole liver under breath holding [54].

\section{Extracellular Fluid Contrast Agents}

Extracellular fluid contrast agents, such as Gd-DTPA, are the first contrast agents intended for MRI and have a high signal enhancement effect. The diagnostic performance of dynamic MRI using extracellular fluid contrast agents is equal to or greater than that of dynamic CT for hypervascular HCCs [55].

\section{RES Contrast Agents}

RES contrast agents contain superparamagnetic iron oxide particles and are used to visualize the abundance and function of Kupffer cells on T2-weighted and T2*weighted images. Overt HCCs lack Kupffer cells and thus appear hyperintense compared with the hypointense background liver on T2-weighted and T2*-weighted images. By contrast, early HCCs contain Kupffer cells, which may reduce the signal intensity, making them difficult to differentiate from dysplastic nodules [56].

\section{Hepatocyte-Specific Contrast Agents}

Gd-EOB-DTPA is a hepatocyte-specific contrast agent that acts as a conventional extracellular fluid contrast agent early after intravenous infusion. This allows assessment of hemodynamics in dynamic studies. Over time, this agent is modified with a lipid-soluble side chain (EOB group) and taken up by hepatocytes via transporters expressed on the hepatocyte cell membrane. T1-weighted images acquired $20 \mathrm{~min}$ after contrast agent administration are referred to as hepatocyte or hepatobiliary phase images. These show the highest performance for detecting small liver lesions because of the high contrast enhancement of the normal liver parenchyma compared with that in overt HCCs, which no longer have normal hepatocyte function and thus have reduced contrast agent uptake [57]. Some advanced HCCs appear hyperintense in the hepatocyte phase, which is associated with the ex- 
pression of the transporter organic anion transporting polypeptide 1B3 (OATPIB3) [24]. These lesions, that is, hyperintense lesions on hepatobiliary phase EOB-MRI, are associated with $\mathrm{WNT} / \beta$-catenin mutation [58] and primary resistance to immune checkpoint inhibitors (ICIs) [59].

Gd-EOB-DTPA is also useful for diagnosing early HCCs, which are identified as hypointense lesions compared with the background liver in the hepatobiliary phase, during which transporter expression decreases before the blood flow changes within the tumor [60]. By contrast, high-flow, small hemangiomas can be difficult to differentiate from hypervascular HCCs because they are enhanced in the early phase and hypointense in the late and hepatobiliary phases; they can be differentiated by signal intensity on T2-weighted images [61].

\section{CTHA and CTAP}

CTHA and CTAP are highly sensitive tools for assessing intranodular blood flow. The abundance of arterial and portal blood flow is associated with the malignancy grade of hepatocellular nodular lesions during the course of multistep hepatocarcinogenesis, and increased arterial blood flow and decreased portal blood flow are associated with increasing malignancy grade [62]. For this reason, CTHA and CTAP are the gold standard and the most sensitive diagnostic tools for detecting overt HCCs and for characterizing hepatocellular nodular lesions [63]. In Japan, integrated CT and angiography systems are frequently used in clinical practice.

Nevertheless, because these tests are relatively invasive, they are performed in increasingly fewer cases and only for diagnostic purposes. The indications for CTHA/ CTAP are limited to determining the definitive diagnosis of lesions that are undiagnosable using noninvasive imaging modalities (ultrasound, CT, or MRI), planning for surgical resection, confirming the absence of intrahepatic residual lesions, and evaluating treatment response at the time of therapeutic procedures such as TACE.

\section{Consensus Statements}

19. Both EOB-MRI and dynamic CT are useful for HCC surveillance and diagnosis, but the former has better performance overall.

20. Because the 2 modalities complement each other, it is important to use them flexibly and to take advantage of their respective characteristics.

21. EOB-MRI has the capacity for assessing liver tumor hemodynamics in the dynamic phase and hepatocyte function in the hepatobiliary phase. It can be combined with other sequences, such as T1-weighted, T2-weighted, and diffusion-weighted images, to improve the accuracy of HCC diagnosis.

22. EOB-MRI can be used for assessing multicentricity and intrahepatic metastasis of HCC, and it is effective for pretreatment disease staging.

23. EOB-MRI is effective for differentiating between dysplastic nodules and early HCC.

24. In patients at high risk of HCC (such as hepatitis Bor hepatitis C-related cirrhotic patients), EOB-MRI should be performed at least once per year as a screening test.

25. EOB-MRI might not distinguish some HCCs from high-flow-type hemangioma.

\section{Imaging Diagnosis of Pathologically Early HCC Consensus Statements}

26. HCC can be classified according to developmental stage into dysplastic nodules/early HCC (well-differentiated carcinoma with indistinct margin and portal tract areas inside), well-differentiated HCC (well-differentiated carcinoma with distinct margin but no portal tract areas inside), and typical HCC (moderately differentiated hepatocellularcarcinoma). There are no typical imaging findings for early HCC, highlighting the importance of comprehensive assessment with different imaging findings.

27. On CECT, which reflects hepatic blood flow, portal flow in early HCCs is equal to or slightly lower than that of the surrounding liver. Hepatic arterial flow in early HCCs is slightly reduced or equal to that of the surrounding liver, which differs from the hemodynamics of overt HCCs.

28. Early HCCs contain Kupffer cells, although their number may be reduced, and they may be visualized as slightly hypoechoic nodules in the Kupffer phase of CEUS.

29. On EOB-MRI, early HCCs are often depicted as hypointense nodules in the hepatobiliary phase.

30. In clinical practice, deciding when to start treatment in each borderline/atypical lesion is more important than differentiating early/well-differentiated HCCs from dysplastic nodules.

\section{CT Findings}

Early HCCs are depicted as nonspecific, hypointense nodules on CT, either plain or contrast-enhanced. On dynamic CECT of the liver, the arterial phase reflects hepatic arterial blood flow and the portal phase reflects portal blood flow. Unlike hypervascular (or overt) HCCs, 
early HCCs do not appear as high-attenuation lesions in the arterial phase because arterial vessels are not increased, and they are not clearly low attenuation compared with the surrounding liver in the portal phase because portal blood flow is still present or only minimally decreased [63]. Consistent with this, the mass is not visualized as an obvious defect on CTAP but appears isoattenuate or slightly hypoattenuate compared with the surrounding liver. Moreover, because intra-tumor arterial blood flow is not increased, the mass does not appear hyperattenuate on CTHA. Instead, intra-tumor arterial blood flow decreases in certain stages of early HCC, and consistent with this, early HCCs can appear hypoattenuate with respect to the surrounding liver on CTHA.

\section{MRI Findings}

Early HCCs show varying signal intensities on T1weighted imaging, although they are typically isointense. Similarly, they are typically isointense on T2weighted and diffusion-weighted imaging. They are visualized as nonspecific lesions and are often unrecognizable on non-contrast MRI. A recent study of hepatic atypical nodules demonstrated that EOB-MRI is useful for detecting early HCC. After intravenous administration, approximately half of the Gd-EOB-DTPA dose is excreted by the kidney and the other half is taken up by hepatocytes and excreted by the biliary system. The hepatocyte membrane transporter OATP1B3 (OATP8) is involved in the uptake of this contrast agent [24, 64], although approximately $85 \%$ of early HCCs have reduced expression of this transporter [65]. This may explain why early HCCs are often depicted as hypointense nodules in the hepatobiliary phase of EOB-MRI. Some dysplastic nodules also appear as hypointense nodules in the hepatobiliary phase of EOB-MRI; however, these nodules are generally smaller than HCC, and their margins are often indistinct. Because differentiating between high-grade dysplastic nodules and early HCCs is difficult, even pathologically, which of these hypointense nodules are early HCCs and what characteristics distinguish dysplastic nodules from early HCCs remains undetermined and warrants further investigation. It should be noted that some HCCs pathologically characterized as early HCCs do not appear hypointense in the hepatocyte phase of EOB-MRI [24].

\section{Early HCC with Severe Fatty Change}

Severe fatty change is common in early HCCs and modifies the imaging findings. These lesions are difficult to differentiate from hypovascular fatty nodules (e.g., fo- cal fatty liver, large regenerative nodules with severe fatty change, borderline lesions, and some well-differentiated HCCs), as they share certain imaging features (e.g., high echogenicity, hyperintensity on T1- and T2-weighted images, and hypointensity on T1 opposed-phase imaging).

\section{Hypovascular Hypointense Nodules}

\section{Consensus Statements}

31. EOB-MRI hepatobiliary phase images are effective for detecting hypovascular hepatocellular nodules in patients at high risk of HCC.

32. Hypovascular nodules measuring $>1 \mathrm{~cm}$ that are hypointense on EOB-MRI hepatobiliary phase images in the HCC high-risk group are likely early HCCs and should undergo tumor biopsy.

33. Hypovascular nodules that are hypointense on EOB-MRI hepatobiliary phase images can grow and potentially progress to hypervascular HCCs, which require therapeutic intervention.

\section{Natural Course of Hypovascular Hypointense}

Nodules

An increasing number of hypovascular nodules measuring approximately $1 \mathrm{~cm}$ that appear hypointense in the hepatobiliary phase of EOB-MRI are detected during follow-up of chronic liver disease, such as hepatitis C or B virus-related cirrhosis. These hypovascular nodules that are hypointense on EOB-MRI hepatobiliary phase images are likely dysplastic nodules or early HCCs $[60,66]$. These hypovascular nodules progress to hypervascular typical HCCs at a high frequency (approximately 15-27\% at 1 year) [67-69], and eventually, $>80 \%$ of these nodules progress to typical hypervascular HCC. Large tumor size and an increasing trend in size are important risk factors for progression to hypervascular HCC [67-69].

Diagnostic Algorithm for the Treatment Indication of Hypovascular Hypointense Hepatocellular

Nodules (Early HCC)

The JSH 2017 edition of the Clinical Practice Guidelines for HCC provides the "HCC surveillance and diagnostic algorithm" for patients at very high or high risk of HCC [9]. For nodules measuring $\geq 1 \mathrm{~cm}$ detected on ultrasound that exhibit an early enhancement pattern on dynamic CT/MRI without "washout" or those showing no early enhancement but measuring $\geq 1.5 \mathrm{~cm}$, tumor biopsy is recommended [9]. Because EOB-MRI has the highest performance for detecting early HCCs $[60,66]$, the 2017 guidelines have added EOB-MRI to the 1st line dynamic study for diagnosing nodules detected by ultra- 
sound, thus increasing the chances of detecting hypovascular hypointense hepatocellular nodules, including early HCCs, on EOB-MRI hepatobiliary phase images.

The diagnostic algorithm and recall policy in cirrhotic liver proposed by the European Association for the Study of the Liver (EASL) [70] and the surveillance/diagnostic algorithm from the American Association for the Study of Liver Diseases (AASLD) [71] also state that nodules that are $\geq 1 \mathrm{~cm}$ in size, hypovascular, and hypointense on EOB-MRI hepatobiliary phase images should be biopsied and, if diagnosed histologically as HCC, subjected to therapeutic intervention. The AASLD's diagnostic algorithm, which incorporates the Liver Imaging Reporting And Data System (LI-RADS) established by the American College of Radiology (ACR), defines hypointensity on EOB-MRI hepatobiliary phase images, the most important imaging feature of hypovascular hepatocellular nodules, as an ancillary feature suggestive of HCC [71].

Takayama et al. [72] compared the overall survival (OS) and recurrence-free survival (RFS) rates of patients undergoing surgery for solitary early and overt HCCs measuring $\leq 2 \mathrm{~cm}$ in size; the OS and RFS rates of early HCC cases were significantly longer than those of overt HCC cases. Pathologically, early HCCs do not undergo intrahepatic metastasis, whereas typical hypervascular HCCs, even those $<2 \mathrm{~cm}$, are associated with portal vein involvement and intrahepatic metastasis, providing a rationale for initiating therapeutic intervention at the stage of early HCC [19]. Midorikawa et al. [73] performed a retrospective analysis of 248 surgical and 28 untreated follow-up cases of solitary early and overt HCCs $\leq 2 \mathrm{~cm}$ in size; they attributed the better survival outcomes of surgically treated early HCC cases to lead time bias. However, because this was a retrospective study with a small untreated follow-up group, further investigation is warranted.

As mentioned above, the JSH Clinical Practice Guidelines for HCC 2017 recommend EOB-MRI as the 1st line dynamic study for diagnosing nodules detected with ultrasound, and this will increase the chances of detecting early HCCs by tumor biopsy and treating them. In comparison, the consensus-based HCC surveillance and diagnostic algorithm (2015 updated version) recommended in the JSH Clinical Practice Manual for HCC [74] states that a hypovascular nodule appearing hypointense on EOB-MRI hepatobiliary phase images and visualized as a defect on Sonazoid ${ }^{\circledR}$ CEUS Kupffer imaging should be diagnosed as HCC and treated without liver biopsy [7476]. This algorithm has been taken over by the Asian Pacific Association for the Study of the Liver Guidelines for HCC (2017 update), and exactly the same surveillance and diagnostic algorithm was adopted and is widely used [77].

Hypovascular nodules detected on EOB-MRI hepatobiliary phase images can be followed up using regular EOB-MRI and/or CEUS [76]; however, they should be treated once nodule-in-nodule appearance is detected. As such, EOB-MRI is important for detecting early HCCs. Although early HCCs should be diagnosed and treated according to the diagnostic algorithm in the Clinical Practice Guidelines for HCC 2017, in cases in which a liver biopsy is difficult to perform, the timing for initiating treatment should be determined according to tumor size and growth speed [67-69] during the close monitoring of such nodules by EOB-MRI [77].

\section{Staging of HCC}

\section{Consensus Statements}

34. The unique TNM classification system of the Liver Cancer Study Group of Japan is used for TNM staging in Japan.

35. Although the Child-Pugh classification is widely used globally for the classification of liver function, "liver damage grade" may be more accurate to determine whether to perform hepatectomy because it includes the indocyanine green retention rate at 15 min (ICG R15).

36 Both the Child-Pugh classification and "liver damage grade" have limitations, including the use of subjective and confounding factors (albumin and ascites) and the need for ICG injection to determine "liver damage grade."

37. The albumin-bilirubin (ALBI) grade, which is statistically established using only albumin and total bilirubin levels, is useful for evaluating patients with good liver functional reserve.

38. The Japan Integrated Staging (JIS) score is useful as an integrated staging system; its variants, including ALBI-TNM (ALBI-T) and modified ALBI-T (mALBI-T), have also been reported.

There are 3 types of staging systems for HCC: (1) TNM classification for tumor staging, (2) classification of liver functional reserve, and (3) integrated staging systems that combine the former 2. For carcinomas, including HCC, the TNM classification describes the degree of tumor extension and has been used in clinical practice for years. However, both tumor progression stage and liver functional reserve need to be evaluated in HCC to determine a treatment strategy and for predicting prognosis. 
Table 6. Integrated staging system for HCC

\begin{tabular}{|c|c|c|c|c|c|c|}
\hline $\begin{array}{l}\text { Integrated staging } \\
\text { system }\end{array}$ & Liver factor & Tumor factor & Other factors & Author & Year & Ref. \\
\hline Okuda classification & Albumin, bilirubin, ascites & Tumor occupation $(\geq 50 \%$ or $<50 \%)$ & - & Okuda & 1985 & [78] \\
\hline CLIP score & Child-Pugh & $\begin{array}{l}\text { Tumor burden } \\
\text { PVTT }\end{array}$ & $\operatorname{AFP}(\geq 400$ or $<400)$ & $\begin{array}{l}\text { CLIP } \\
\text { investigators }\end{array}$ & 1998 & {$[81]$} \\
\hline JIS score & Child-Pugh & TNM by LCSGJ & - & Kudo & 2003 & {$[82]$} \\
\hline m-JIS score & $\mathrm{LD}$ & TNM by LCSGJ & - & Nanashima & 2004 & {$[84]$} \\
\hline bm-JIS score & Child-Pugh & TNM by LCSGJ & AFP, AFP-L3, DCP & Kitai & 2008 & {$[86]$} \\
\hline Tokyo score & Albumin, bilirubin & Tumor size, tumor number & - & Tateishi & 2005 & {$[87]$} \\
\hline BALAD score & Albumin, bilirubin & - & AFP, AFP-L3, DCP & Toyoda & 2006 & {$[88]$} \\
\hline BALAD-2 score & Albumin, bilirubin & - & AFP, AFP-L3, DCP & Toyoda & 2017 & {$[89]$} \\
\hline GALAD score & - & - & AFP, AFP-L3, DCP, age, gender & Caviglia & 2016 & {$[90]$} \\
\hline ALBI-T score & ALBI & TNM by LCSGJ & - & Hiraoka & 2016 & {$[91]$} \\
\hline mALBI-T score & mALBI & TNM by LCSGJ & - & Hiraoka & 2019 & {$[92]$} \\
\hline
\end{tabular}

HCC, hepatocellular carcinoma; PVTT, portal vein tumor thrombosis; LCSGJ, Liver Cancer Study Group of Japan; TNM, tumor-node-metastasis classification; CP, Child-Pugh class; LD, liver damage by LCSGJ; JIS, Japan Integrated Staging; m-JIS, modified JIS; bm-JIS, biomarker combined JIS; AFP, alpha-fetoprotein; AFP-L3, fucosylated AFP; DCP, des-r-carboxy prothrombin; BALAD, bilirubin, albumin, AFP-L3, AFP, and DCP model; ALBI, albuminbilirubin grade; ALBI-T, ALBI-TNM; mALBI, modified ALBI grade; mALBI-T, mALBI-TNM.

\section{Integrated Staging Systems}

The combination of liver function classification and TNM classification is an integrated staging system that includes Okuda stage [78], Barcelona Clinic Liver Cancer (BCLC) stage $[79,80]$, Cancer of the Liver Italian Program (CLIP) score [81], JIS score [82, 83], modified JIS score [84, 85], biomarker JIS score [86], Tokyo score [87], BALAD (based on 5 serum markers: bilirubin, albumin, AFP-L3, AFP, and DCP) score [88], BALAD2 score [89], GALAD (gender, age, AFPL3, AFP, and DCP) score [90], ALBI-T score [91], and mALBI-T score [92] (Table 6). Among these, the JIS score, which combines the TNM classification of the Liver Cancer Study Group of Japan and the ChildPugh classification, has the highest utility and versatility as an overall staging tool for HCC patients. One of the advantages of the JIS score is that each score is equally stratified. The increase in the proportion of HCC patients with good liver functional reserve has increased the expectations regarding the ALBI-T/ mALBI-T score, a variant of the JIS score that uses ALBI and mALBI grades, and can identify HCC patients with relatively good liver function with high accuracy, as mentioned earlier [92].

\section{Significance of Integrated Staging Systems}

The clinical significance of integrated staging systems can be summarized as follows:

1. Accurate prognostic prediction for an individual: TNM staging alone is inadequate for predicting the prognosis of HCC patients whose liver function affects the treatment outcome. Therefore, the integration of TNM and liver function is essential for accurate prognostic prediction.

2. Establishing a common scale for selecting the most appropriate treatment for an individual score or stage.

3. Identifying patient populations that should be treated curatively.

4. Identifying patient populations in which treatment may lead to poor outcomes.

5. Establishing a common scale for comparing outcomes between treatments and between different centers. Although simple comparisons between treatments are difficult to make, integrated staging systems are useful for comparing the outcomes of certain treatment modalities (e.g., surgery, local ablation therapy, and TACE) at the same score using the same scoring system. 
Future Perspectives of the Staging Systems

Currently, the BCLC staging system is widely used outside Japan. However, the BCLC system is a treatment selection staging method intended for decision-making purposes, whereas the JIS score (and its variants) and the CLIP score are prognostic predictive staging systems. The Hong Kong Liver Cancer (HKLC) staging system $[93,94]$ has also been reported, but its usefulness remains inconclusive.

In Japan, where surveillance systems are widely used, there are more opportunities to detect small HCCs that can be treated curatively, for which the JIS score and its variants are highly effective. However, in countries where early detection of HCC is limited, the CLIP score and the BALAD score, which can predict prognosis based on blood tests alone [88], may be more effective staging systems or biomarkers.

Although various staging systems have been proposed for the integrated staging system of liver function and TNM staging in the clinical management of HCC, 3 requirements must be met for their use in clinical practice: (1) simple and easy to remember, (2) associated with fewer missing data required for analysis, and (3) good performance in stratifying patients from normal liver function to severe liver dysfunction, and from early-stage to advanced or late-stage disease. The ICG test is commonly performed in patients considered for resection; therefore, those with available data for ICG R15 account for only $66.4 \%$ of all analyzable cases in the database of the Liver Cancer Study Group of Japan $[95,96]$. In this regard, the JIS, ALBI-T, and mALBI-T scores are effective for retrospectively evaluating treatment outcomes and are staging systems suitable for the clinical situation in Japan.

\section{Surveillance and Diagnostic Algorithm of HCC Recommended by JSH}

\section{Consensus Statements}

39. If a lesion is hypervascular in the arterial phase of CECT or MRI and shows low attenuation/intensity compared with the surrounding liver parenchyma ("washout") in the portal/equilibrium phase, a diagnosis of typical HCC can be made.

40. If a lesion is not visualized as hypervascular in the arterial phase of dynamic CT/MRI but is smaller than $1.5 \mathrm{~cm}$ in diameter and can be visualized on ultrasound, the patient should be followed up with ultrasound every 3 months. If tumor size increases or any tumor marker is elevated, dynamic CT/MRI needs to be repeated.
41. If the lesion cannot be visualized on ultrasound, follow-up with dynamic CT/MRI should be considered. Follow-up is not required for lesions that are confirmed to be benign on CT/MRI.

42. Lesions visualized as hypervascular areas in the arterial phase of dynamic CT and measuring $\geq 1.5 \mathrm{~cm}$ should be subjected to EOB-MRI. For lesions appearing hypointense in the hepatobiliary phase, liver tumor biopsy, CEUS, superparamagnetic iron oxide-enhanced MRI, or CTAP/CTHA should be considered (Fig. 2).

\section{Treatment of HCC}

\section{Surgery}

\section{Consensus Statements}

43. Hepatectomy is indicated when the tumor is confined to the liver and in $\leq 3$ tumors.

44. There is no restriction regarding the size of the tumor, and cases with portal vein invasion up to the 1st branch may be considered eligible for surgery.

45. Hepatectomy is the most curative treatment for HCC and is recommended for patients with ChildPugh class A or B liver function.

46. Hepatectomy is recommended for a solitary tumor of any size and as the first choice of treatment for tumors $>3 \mathrm{~cm}$ in diameter.

47. The safety of hepatectomy has been established, with an operative mortality rate of $1.4 \%$.

48. For small HCCs, anatomical resection or partial resection (in cases of poor liver function) is the treatment of choice.

49. The major factors affecting the postoperative outcome of hepatectomy are tumor size, number of tumors, vascular invasion, and liver function.

Hepatectomy is one of the standard treatments for HCC and is the most curative treatment for this disease. The safety of hepatectomy has dramatically improved in recent years; an analysis using the National Clinical Database revealed a $1.4 \%$ overall operative mortality rate for hepatectomy (as of 2016) [97]. To further improve safety, it is necessary to accurately assess the degree of HCC progression and the patient's liver function to identify eligi-

Fig. 2. Surveillance and diagnostic algorithm of HCC. HCC, hepatocellular carcinoma; CT, computed tomography; MRI, magnetic resonance imaging; CTAP, CT during arterioportography; CTAH, CT during hepatic arteriography; US, ultrasound. Reproduced with permission from the Japan Society of Hepatology [8], Kokudo et al. [9], and the Japan Society of Hepatology [17].

(For figure see next page.)
Liver Cancer 2021;10:181-223 DOI: $10.1159 / 000514174$
Kudo et al. 


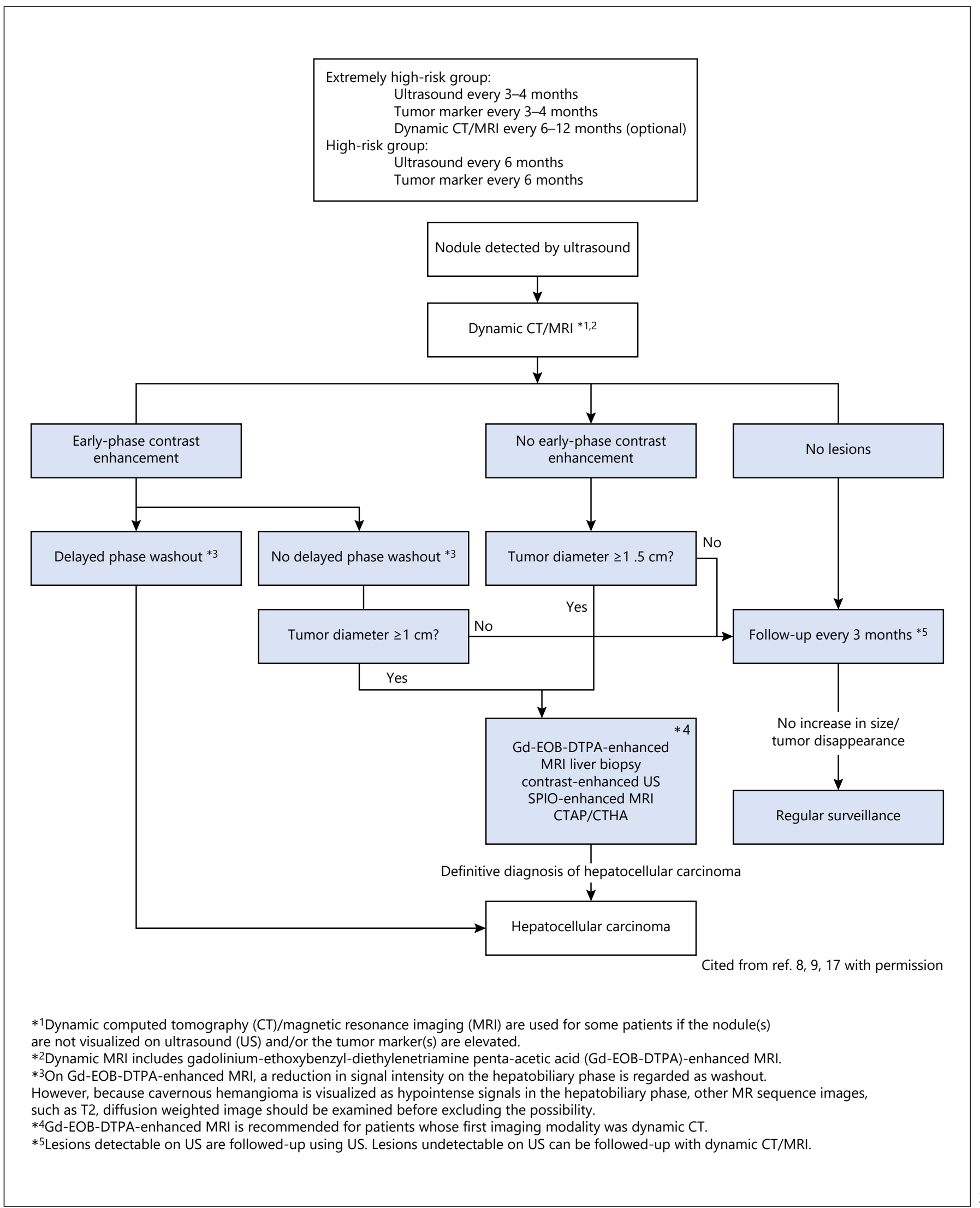

JSH Consensus Statements and Recommendations on HCC
Liver Cancer 2021:10:181-223

DOI: $10.1159 / 000514174$ 
ble patients and to select a safe and rational surgical technique based on these assessments [9].

In Western countries, patients classified as ChildPugh class $\mathrm{B}$ or $\mathrm{C}$ and those with portal hypertension are considered ineligible for hepatectomy even in early-stage HCCs (BCLC stage A). Even in Child-Pugh A cases, liver transplantation is recommended if there is portal hypertension. Although liver transplantation is theoretically more curative than hepatectomy, in Japan, it is more appropriate to select hepatectomy as the first choice for resectable HCCs for 2 reasons: the relatively good shortterm postoperative outcomes with hepatectomy and the difficulty in performing brain-death liver transplantation. Ishizawa et al. [98] demonstrated the safety of minor hepatectomy in HCC patients with portal hypertension. In Western countries, an increasing number of guidelines also allow minor resection in some patients with portal hypertension [70]. The 2017 revised edition of JSH Clinical Practice Guidelines for HCC published in October 2017 recommends hepatectomy in all Child-Pugh A/B cases with no $>3$ tumors regardless of tumor size [9].

\section{Ablation}

\section{Consensus Statements}

50. Radiofrequency ablation (RFA) is generally indicated in Child-Pugh class A/B patients with tumor size $\leq 3 \mathrm{~cm}$ and $\leq 3$ tumors. Patients with vascular invasion or extrahepatic metastasis are generally considered ineligible.

51. RFA should be performed with adequate precautions in patients with low platelet count, impaired coagulation function, ascites, gastrointestinal adhesions, bile duct-to-intestinal anastomosis or endoscopically incised duodenal papilla, tumor located close to the Glissonean sheath (bile duct) in the hepatic hilum, and complications of renal failure.

52. The expansion of indications for RFA to include intermediate-stage and large HCCs is also under investigation.

53. For HCCs protruding on the liver surface or those abutting on adjacent organs, the laparoscopic approach should be considered to avoid risk of damage to other organs.

54. Artificial pleural effusion/ascites techniques are mainly used in cases in which the lesion is located immediately below the diaphragm and cannot be visualized on ultrasound because of lung air.

55. When there is a risk of gastrointestinal perforation or diaphragmatic injury, RFA with the artificial ascites method should be considered.
56. For HCCs that cannot be visualized on B-mode ultrasound, CEUS-guided RFA or fusion image-guided RFA is useful to successfully treat the tumor.

57. When a poorly defined tumor or multiple defects including previously ablated HCCs are detected by CEUS-guided RFA, Sonazoid ${ }^{\circledR}$ reinjection at the Kupffer phase can visualize the viable tumor and confirm its location (Defect Reperfusion Imaging: DRPI).

58. Bipolar RFA is most effective when used in the multipolar mode, which uses multiple electrodes, allowing for no-touch ablation, free puncture needle navigation to avoid large vessels and bile ducts, and complete ablation of large HCCs in a short time.

\section{Important Clinical Reports of RFA}

Shiina et al. [99] conducted a randomized controlled trial (RCT) to compare treatment outcomes between RFA and percutaneous ethanol injection therapy (PEIT). The 4-year survival was significantly better in the RFA group (74\%, 95\% confidence interval $[\mathrm{CI}]: 65-84)$ than in the PEIT group (57\%, 95\% CI: 45-71; $p=0.01)$. Similarly, the RFA group had significantly better RFS and local recurrence rates, with no significant differences in complications between the 2 groups. Given these results, the authors concluded that RFA is superior to PEIT.

Chen et al. [100] conducted an RCT to compare the long-term outcome of RFA with that of hepatectomy for small solitary HCCs. A total of 180 patients with solitary HCC $\leq 5 \mathrm{~cm}$ diameter were randomized to undergo either RFA or hepatectomy ( $n=90$ each). The 4-year survival rate was $67.9 \%$ with RFA and $64.0 \%$ with hepatectomy, with no significant difference between the groups. The 4-year RFS was $67.9 \%$ with RFA and $64.0 \%$ with hepatectomy, again with no significant difference. When patients in each group were divided into $<3$ and $\geq 3 \mathrm{~cm}$ tumor groups, OS and RFS did not differ significantly between the 2 groups.

Huang et al. [101] conducted an RCT to compare the long-term outcomes of hepatectomy with those of RFA for small HCCs. A total of 230 patients with HCC who met the Milan criteria were randomized to the hepatectomy $(n=115)$ and RFA $(n=115)$ groups. The 5-year survival rate was significantly lower in the RFA group $(54.78 \%)$ than in the hepatectomy group $(75.65 \%$; $p=$ $0.001)$. The 5-year RFS was also significantly lower in the RFA group $(28.69 \%)$ than in the hepatectomy group $(51.30 \% ; p=0.017)$. By contrast, the incidence of serious complications and the number of days in the hospital were significantly lower in the RFA group $(p<0.05)$. The authors concluded that hepatectomy was associated with 
a better prognosis and lower recurrence rate than RFA in HCC patients meeting the Milan criteria.

Izumi et al. [102] presented the results of the "Efficacy of surgery versus radiofrequency ablation on primary hepatocellular carcinoma: a multicenter randomized controlled trial (SURF trial)" at the Annual Meeting of the American Society of Clinical Oncology (ASCO) in 2019. This RCT was conducted to provide evidence from Japan. The trial involved patients with HCC with good liver function (Child-Pugh score $\leq 7$ ), tumor size $\leq 3 \mathrm{~cm}$, and $\leq 3$ tumors (150 treated with hepatectomy and 151 with RFA). The median (95\% CI) observation period was 5.04 (0.36-9.49) years in the hepatectomy group and $4.99(0.00-8.70)$ years in the RFA group, with a median (95\% CI) RFS of 2.98 $(2.33-3.86)$ and $2.76(2.17-3.80)$ years, respectively $(p=$ 0.793 ). The 3 -year RFS was $49.8 \%$ in the hepatectomy group and $47.7 \%$ in the RFA group, with no significant difference.

\section{Expanded Indications for RFA}

Because there is no clear difference in the treatment outcome of RFA according to tumor size (e.g., $<$ or $\geq 3 \mathrm{~cm}$ ) or the number of tumors (e.g., $<$ or $\geq 3$ tumors), attempts are being made to extend the indications for RFA. Nouso et al. [103] conducted a retrospective propensity score-matched study to compare RFA and TACE in patients with intermediate-stage HCC. After matching for 18 factors, including tumor size, number of tumors, and background liver function, the authors noted significantly better outcomes with RFA than with TACE. In particular, the outcomes were better for RFA than for TACE in patients classified as Bolondi subclass B1 or B2 [104], suggesting that RFA should be considered even in patients with intermediate-stage HCC.

In clinical practice, multiple nodules with different sizes can be ablated separately using an adjustable electrode. This eliminates the barriers to the expansion of indications for RFA to include HCC with $\geq 4$ tumors.

Yin et al. [105] examined the outcome of RFA versus microwave coagulation therapy (MCT) in 109 HCC patients with at least 1 nodule measuring $3-7 \mathrm{~cm}$ (main tumor diameter: $3-5 \mathrm{~cm}$ in 89 and $5-7 \mathrm{~cm}$ in 20 patients; 59 treated with RFA and 50 with MCT). Complete ablation was achieved in $92.6 \%$ of patients. The local recurrence rate was $22 \%$ (median time to recurrence: 4.6 months). The 1-, 3-, and 5-year survival rates were $75.8,30.9$, and $15.4 \%$, respectively. Based on these results, the authors concluded that RFA and MCT were effective and safe for large nodules.

\section{Complications}

The treatment-related mortality rate for RFA ranges from 0.1 to $0.3 \%$, with the incidence of serious complica- tions ranging from 3.0 to $7.8 \%$. The most common complications include intra-abdominal hemorrhage $(0.2-$ $0.5 \%)$, biliary hemorrhage $(0.08 \%)$, hemothorax $(0.2-$ $0.8 \%)$, pneumothorax $(0.2 \%)$, hepatic infarction $(0.2-0.5 \%)$, liver tumor $(0.2-0.8 \%)$, gastrointestinal perforation $(0.3-0.5 \%)$, dissemination $(0.2-0.8 \%)$, portal vein thrombosis $(0.2 \%)$, and bile leaks/bile duct dilatation $(0.2-1.0 \%)[96,106-109]$.

\section{Transarterial Chemoembolization}

Consensus Statements

59. TACE is mainly indicated in patients with hypervascular HCCs classified as Child-Pugh class A or B, with 2-3 tumors of $\geq 3 \mathrm{~cm}$ in diameter or $\geq 4$ tumors, and may be indicated for some patients with minor vascular invasion.

60. For localized tumors, superselective infusion of Lipiodol $^{\circledR}$ mixed with anticancer agents followed by gelatin sponge injection with visualization of portal branch improves the local treatment efficacy.

61. Whether concomitant use of anticancer agents and embolization improves the efficacy of TACE and whether there is a difference in TACE efficacy according to drug type remain unknown.

62. More superselective (ultraselective) embolization will improve local control rate and OS and reduce complications (curative TACE).

Conventional TACE (CTACE) was developed in the late 1970s in Japan $[110,111]$ and has been widely used as an effective treatment for unresectable HCC. According to the 20th Nation-wide Follow-up Survey Report on Primary Liver Cancer [96], cTACE using Lipiodol ${ }^{\circledR}$ and gelatin sponge was performed as a first-line treatment in $27.5 \%(n=5,083)$ of 18,458 initially diagnosed HCC cases and $39.3 \%(n=2,627)$ of 6,687 recurrent cases of HCC, making it the most commonly used treatment for HCC [96]. Although TACE using drug-eluting beads (DEB) was introduced in Japan in 2014, cTACE remains the mainstream treatment for HCC, and the improved outcomes of cTACE have directly translated into prolonged survival of HCC patients. Because the response to treatment with cTACE depends on the operator's skill, full understanding of the principles of cTACE is important to ensure safe and effective therapy.

\section{Conventional TACE}

Principles of cTACE

Hepatic arteries are the main blood supply in HCC; however, areas of extracapsular invasion, well-differentiated HCC areas, and adjacent satellite nodules are also 
supplied by the portal venous flow. Moreover, after arterial chemoembolization has been achieved, portal blood flow comes into the viable tumor lesion through the tumor's draining portal vessel and surrounding sinusoids, thus allowing the tumor to survive. Therefore, local recurrence of HCC occurs at a relatively high frequency after arterial embolization alone. Lipiodol ${ }^{\circledR}$, an oil-based iodinated contrast agent, is a semiliquid embolization material that remains in the tumor vasculature after superselective injection from an artery. Subsequently, part of the agent passes through the tumor's drainage portal vessel and portal vein through the peribiliary plexus, which temporarily embolizes both the artery and portal branches around the tumor (temporal liver infarction). After injection of Lipiodol ${ }^{\circledR}$ mixed with anticancer agents, a gelatin sponge is introduced to embolize the artery, thereby achieving simultaneous arterial and portal embolization; this leads to complete necrosis of the tumor and satellite lesions in the surrounding liver parenchyma [112-114]. Thus, cTACE is highly effective in blocking the blood supply from both the artery and portal vein to the tumor, although it can also minimally damage the surrounding liver parenchyma.

\section{Indications for TACE}

While TACE is mainly indicated for patients with hypervascular HCCs with background liver function of Child-Pugh class A or B, 2-3 tumors measuring $>3 \mathrm{~cm}$ in diameter, or $\geq 4$ tumors, the JSH Clinical Practice Guidelines for HCC 2017 have extended the indications to include tumors associated with vascular invasion [9]. However, a European RCT showed that the survival outcome of cTACE-treated patients with BCLC stage B (BCLC-B) HCC and Child-Pugh scores $\geq 9$ is worse than that of the best supportive care (BSC) group [115], suggesting that these patients should be considered ineligible for CTACE. In patients classified as Child-Pugh class C, cTACE is still recommended in those $<65$ years of age, who meet the Milan criteria (a single tumor $\leq 5 \mathrm{~cm}$ diameter or $\leq 3 \mathrm{tu}$ mors of $\leq 3 \mathrm{~cm}$ diameter) $[114,116-121]$. cTACE is also widely used as pretreatment for RFA because it extends the ablation volume and because Lipiodol ${ }^{\circledR}$ clarifies the contour of the tumor, which makes it easier to determine the ablative margin on CT and enables accurate assessment of the treatment response after RFA.

\section{Different Indications of DEB-TACE and cTACE}

Consensus Statements

63. For small HCCs that are curable by superselective TACE, cTACE is theoretically more effective than
DEB-TACE because it can necrotize the peritumoral area including capsular invasion through a portal venous flow block around the tumor.

64. DEB-TACE may be more advantageous for patients with bilobar multiple HCCs and poor liver function, those of extremely high age, and those with poor performance status (PS) because it is associated with milder adverse events (AEs).

65. DEB-TACE is preferred for large HCCs. If cTACE is selected, split TACE technique should be carried out or combined with bland transarterial embolization without Lipiodol.

A randomized, phase 2 multicenter trial comparing DEB-TACE and cTACE in 212 patients in 19 centers in 5 European countries (PRECISION V trial) was reported in 2010 [115]. There was no significant difference in tumor response at 6 months, the primary endpoint, between the 2 groups $(p=0.11)$. No significant difference in serious AEs was observed between the groups $(p=0.11)$. In subgroup analyses, however, DEB-TACE was associated with significantly higher response rates in patients with a more severe condition, such as those with Child-Pugh class B liver function, ECOG-PS $=1$, or bilobar multifocal disease. In addition, it was associated with significantly fewer serious AEs. The DC-Bead ${ }^{\circledR}$ used in this study had a bead diameter of $300-500$ or $500-700 \mu \mathrm{m}$, which is larger than the $100-300 \mu \mathrm{m}$ beads commonly used in Japan. In addition, in $27.8 \%$ of patients treated with cTACE, infusion of a Lipiodol ${ }^{\circledR}$-doxorubicin emulsion was not followed by additional gelatin sponge particles or other embolization materials.

The results of the PRECISION Italy multicenter trial were published in 2014. Tumor size and the superselective TACE techniques used were consistent with those in Japan [122]. Although the trial started with a planned sample size of 214 patients, it was completed after 177 patients were enrolled; there was no significant difference in the primary endpoint of OS ( $p=0.949)$. There were no significant differences in the secondary endpoints, including response rate and progression-free survival (PFS), except for a significantly higher frequency of pain after TACE in the cTACE group.

The most recent meta-analysis, which was reported in 2016, included 1,449 patients from 4 RCTs and 8 observational studies [123]. The results showed no significant difference between DEB-TACE and cTACE in terms of response rate, $\mathrm{OS}$, or AEs.

Most recently, Ikeda et al. [124] reported the JIVROSG-1302 PRESIDENT study at ASCO 2020. This RCT clearly showed CR rate at 1 month was much higher in 
Table 7. Clinical trials of TACE combination or with molecular targeted agents

\begin{tabular}{|c|c|c|c|c|c|c|}
\hline & Post-TACE [131] & SPACE [132] & TACE-2 [133] & TACTICS [130] & ORIENTAL [134] & BRISK-TA [135] \\
\hline Phase & 3 & 2 & 3 & 2 & 3 & 3 \\
\hline Design & RCT (vs. placebo) & RCT (vs. placebo) & RCT (vs. placebo) & RCT (open label) & RCT (vs. placebo) & RCT (vs. placebo) \\
\hline Drug & Sorafenib & Sorafenib & Sorafenib & Sorafenib & Orantinib & Brivanib \\
\hline Child-Pugh class & A & A (exclude ascites) & A & $\mathrm{A} 5 \sim \mathrm{B} 7$ & $\mathrm{~A}$ & A \\
\hline ECOG-PS & $0 \sim 1$ & 0 & $0 \sim 1$ & $0 \sim 1$ & $0 \sim 1$ & $0 \sim 1$ \\
\hline \multirow{2}{*}{$\begin{array}{l}\text { BCLC stage } \\
\text { tumor status }\end{array}$} & BCLC stage B & BCLC stage B & BCLC stage B & BCLC stage B & BCLC stage B & BCLC stage B \\
\hline & $\begin{array}{l}\leq 7 \mathrm{~cm}, \leq 10 \\
\text { nodules }\end{array}$ & Unresectable & $\begin{array}{l}\text { Unresectable } \\
\text { Untransplantable }\end{array}$ & $\leq 10 \mathrm{~cm}, \leq 10$ nodules & Unresectable & $\begin{array}{l}\geq 5 \mathrm{~cm} \geq 1 \text { or } \geq 4 \\
\text { nodule }(1 \text { nodule } \geq 3 \\
\mathrm{cm})\end{array}$ \\
\hline TACE & Lp TACE & DEB-TACE & DEB-TACE & Lp TACE & Lp TACE & Any \\
\hline TACE schedule & na & Scheduled & na & On demand & On demand & On demand \\
\hline Primary endpoint & TTP & TTP & PFS & PFS/OS & OS & OS \\
\hline $\begin{array}{l}\text { Response } \\
\text { evaluation }\end{array}$ & RECICL 2004 & mRECIST & RECIST 1.1 & $\begin{array}{l}\text { RECICL } 2009 \text { (intrahepatic } \\
\text { new lesion: not PD) }\end{array}$ & RECIST 1.1 & mRECIST \\
\hline Final result & Negative & $\begin{array}{l}\text { Negative (statistically } \\
\text { positive as phase 2) }\end{array}$ & Negative & Positive & Negative & Negative \\
\hline
\end{tabular}

TACE, transarterial chemoembolization; RCT, randomized controlled trial; BCLC, Barcelona Clinic Liver Cancer; PS, performance status; Lp, Lipiodol ${ }^{\circledR}$; TTP, time to progression; PFS, progression-free survival; OS, overall survival; NA, not applicable.

selective cTACE with epirubicin group $(n=101)$ than that in selective DEB-TACE with epirubicin group $(n=$ 94) (84.2 vs. $35.7 \%, p<0.0001$, odds ratio 7.30 [95\% CI: 2.68-19.89]). Similarly, CR rate at 3 months in cTACE group was $75.2 \%$ as compared with $27.6 \%$ in DEB-TACE group ( $p<0.0001$, odds ratio 8.44 [95\% CI: 4.46-15.96]) [124]. These data strongly suggest that efficacy of selective cTACE conducted in Japan is superior to that of selective DEB-TACE. However, the frequencies of postembolization syndrome were higher in cTACE group than those in DEB-TACE group [124].

\section{Combination Therapy with TACE plus Molecular Targeted Agents \\ Consensus Statements}

66. The combination of TACE with molecular targeted agents (MTAs) may reduce tumor progression after TACE and prolong the interval between TACE sessions, thereby preserving liver function.

67 . When an intrahepatic lesion progresses or a new intrahepatic lesion appears during molecular targeted therapy, additional TACE can be performed to con-

JSH Consensus Statements and Recommendations on HCC trol such lesions and continue molecular targeted therapy.

68. After reduction in the size and/or number of tumors (downstaging) is achieved using TACE plus molecular targeted therapy, additional TACE can be performed to achieve complete response.

Rationale for Combination Therapy with TACE plus MTAs

The rationale for combining TACE with MTAs is based on the following (Fig. 4):

1. Antitumor effects of MTAs themselves: MTAs exert antitumor effects (inhibition of angiogenesis and tumor growth) on tumors left untreated by TACE, thereby preventing the progression of residual tumors and the development of new intrahepatic lesions, vascular invasion, and distant metastasis.

2. Effects of MTAs on tumor vasculature: MTAs act on tumor vasculature to improve vascular permeability, thereby reducing intratumoral interstitial pressure, improving drug delivery, and enhancing the therapeutic effect of TACE [125-128]. 
3. Inhibition of the cytokine storm and the associated tumor progression: Immediately after TACE, hypoxia and the subsequent activation of HIF- $1 \alpha$ cause a surge in vascular endothelial growth factor (VEGF) levels, which may contribute to tumor progression [129]. The use of MTAs (anti-VEGF inhibitors) before or immediately after TACE suppresses this surge and prevents tumor progression.

These mechanisms are suggested to underlie the role of MTAs in combination with TACE. Concomitant use of MTAs can enhance the efficacy of TACE and suppress the progression of residual tumors and the appearance of new intrahepatic lesions, thereby prolonging the interval between TACE sessions [130]. It can also reduce the total number of TACE sessions a patient receives throughout their lifetime, thereby mitigating the decline of liver functional reserve. Concomitant use of MTA with TACE may also prolong patient survival by suppressing the development of vascular invasion, extrahepatic spread, and progression of disease stage.

\section{Results of the TACTICS Trial}

Various clinical trials have been conducted based on the above concepts, although the results are not encouraging [131-135]. One exception is the TACTICS trial conducted in Japan [130]. The positive results obtained in this study are the only positive evidence supporting the efficacy of TACE in combination with MTAs [130] (Table 7).

The TACTICS trial was a randomized, open-label phase 2 trial comparing sorafenib plus cTACE with TACE alone. The primary endpoint of median PFS was 25.2 months in the TACE plus sorafenib group and 13.5 months in the TACE-alone group (hazard ratio [HR]: 0.59 ; 95\% CI: $0.41-0.87 ; p=0.006$ ), demonstrating a significant improvement of PFS with the concomitant use of sorafenib compared with that for TACE alone. The median time to extrahepatic spread or vascular invasion was 22.5 months in the TACE plus sorafenib group and 6.3 months in the TACE-alone group (HR: 0.31 ; $95 \%$ CI: $0.15-0.63 ; p=0.001)$, suggesting that sorafenib significantly reduces the incidence of extrahepatic spread or vascular invasion. The median interval between TACE sessions was 21.1 weeks in the TACE plus sorafenib group and 16.9 weeks in the TACE-alone group ( $p=0.018)$, demonstrating that sorafenib significantly prolongs the interval between TACE sessions [130].
Concept of TACE Failure/Refractoriness and

Treatment Strategy

Consensus Statements

69. TACE failure/refractoriness is defined as follows:

1. Intrahepatic lesions

i. Residual enhancement $(\geq 50 \%)$ of treated nodules observed on response evaluation CT/MRI images obtained $1-3$ months after $\geq 2$ consecutive TACE sessions (despite changing chemotherapeutic agent or reanalyzing the feeding artery).

ii. Increased number of intrahepatic lesions from the previous TACE session observed on response evaluation CT/MRI images obtained 1-3 months after $\geq 2$ consecutive TACE sessions (despite changing chemotherapeutic agent, changing embolization material, or reanalyzing the feeding artery or device).

2. Tumor markers

No decrease in tumor marker level is observed immediately after TACE, or only a minimal and transient decrease is observed after TACE, immediately followed by an increasing trend.

3. Development of vascular invasion.

4. Development of extrahepatic spread.

70. Hepatic arterial infusion chemotherapy (HAIC) is often ineffective in cases of TACE failure/refractoriness; therefore, switching to molecular targeted therapy in these cases is preferable.

71. Molecular targeted therapy is indicated for patients with Child-Pugh class A liver function. It is therefore important to carefully evaluate the indications for TACE and appropriately identify TACE failure/ refractoriness to prevent deterioration of liver function caused by ineffective TACE.

\section{Why Do We Need to Define TACE Failure/}

Refractoriness?

TACE was developed in Japan and has been widely used worldwide. It is recognized as a standard treatment for patients with HCC with liver function classified as Child-Pugh class A or B and multiple intrahepatic lesions without vascular invasion or extrahepatic spread. Although the therapy achieves high local tumor control, its repeated application results in a gradual decrease in liver function, along with a reduced tumor necrotic effect, persistent increase in tumor markers, and the emergence of new lesions. Although these issues are recognized as limitations of TACE, repeated TACE is unavoidable because of the lack of available post-TACE treatments such as 
Table 8. Criteria of TACE failure/refractoriness

1 Intrahepatic lesion

Two or more consecutive ineffective responses within the treated tumors (viable lesion $>50 \%$ ), even after changing the chemotherapeutic agents and/or reanalysis of feeding artery, on response evaluation CT/MRI after 1-3 months following adequately performed selective TACE

Two or more consecutive progressions in the liver (including an increase in the number of tumors compared with that before the previous TACE procedure), even after changing the chemotherapeutic agents and/or reanalysis of feeding artery, on response evaluation CT/MRI after 1-3 months following adequately performed selective TACE

2 Tumor marker

Continuous elevation of tumor markers right after TACE even though transient minor reduction is observed

3 Appearance of vascular invasion

4 Appearance of extrahepatic spread

TACE, transarterial chemoembolization; CT, computed tomography; MRI, magnetic resonance imaging.

MTAs. Given that an effective posttreatment option with MTAs is now available, TACE should not be repeated when refractoriness occurs. This underscores the importance of properly defining TACE failure/refractoriness (Table 8).

Repeated TACE in a patient who has become refractory to TACE leads to impaired liver function and consequently poor prognosis $[136,137]$. However, switching to sorafenib after refractoriness to TACE is more likely to preserve liver function and reduce the incidence of events associated with disease progression, such as extrahepatic or vascular invasion $[136,137]$. The noninterventional, prospective, observational OPTIMIS study reported that patients who switched to sorafenib immediately after developing refractoriness to TACE had a better prognosis than those who continued TACE [138]. Thus, the concept of TACE failure/refractoriness is important to avoid worsening liver function caused by repeated sessions of ineffective TACE in TACE failure/refractory patients (Table 8).

Another first-line agent, lenvatinib, is associated with a markedly high response rate of $40.6 \%$ according to the modified response evaluation in solid tumors response criteria [139], achieving a $61 \%$ response rate in BCLC stage B patients eligible for TACE [140]. The proportion of patients responsive to TACE was $40 \%$ in the OPTIMIS study and $42 \%$ in the BRISK-TA study (TACE-alone arm) [135]. The response rates to lenvatinib in the area of $40 \%$ suggest that lenvatinib is approximately equivalent to TACE in terms of antitumor efficacy. The introduction of lenvatinib for the treatment of patients with poor liver functional reserve resulted in lower response rates, sug-

JSH Consensus Statements and Recommendations on HCC gesting that lenvatinib should be used in patients with relatively good liver function [141]. Because MTAs with high response rates are currently available, refractoriness to TACE should be carefully assessed to avoid worsening of liver function, which in turn will maximize the efficacy of the MTAs.

Criteria for TACE Failure/Refractoriness and Timing of Switching to Molecular Targeted Therapy

In 2011 and 2014, the world's first TACE failure/refractoriness criteria were published by JSH $[13,16]$. Various other criteria have since been published, although the definition of TACE failure/refractoriness itself differs from country to country. The major criteria currently available include (1) the JSH TACE failure/refractoriness criteria [16], (2) the criteria proposed by Raoul et al. [142], (3) the Taiwan criteria, and (4) the International Expert Panel (EPOI HCC) criteria [143]. The JSH criteria define TACE failure/refractoriness as failure to control the tumor in the target lesion or the appearance of new lesions after $\geq 2$ consecutive TACE sessions; the criteria proposed by Raoul et al. [142] also recommend switching to the next treatment if there is no response after 2 TACE sessions. The EPOI HCC criteria and the Taiwan criteria state that patients who need 2 or 3 TACE sessions within 6-12 months should be considered TACE failure/refractory, at which point they should be switched to MTAs. In Taiwan, this is also a criterion for eligibility for insurance reimbursement.

Two retrospective studies clearly showed that switching to sorafenib once the JSH criteria have been met is more beneficial than continuing ineffective TACE thera- 




Fig. 3. Treatment strategy after TACE failure/refractoriness. TACE, transarterial chemoembolization; MTA, molecular targeted agents; HAIC, hepatic arterial infusion chemotherapy. * BSC, best supportive care. ${ }^{+}$HAIC or ablation may be a choice of treatment. Reproduced with permission from the Japan Society of Hepatology [17].

py regarding the preservation of liver function and prolonged time to advanced disease, ultimately leading to prolonged survival $[136,137,144]$. The results of the OPTIMIS study, a prospective, international, noninterventional study designed to verify the results of the 2 retrospective studies $[137,144]$, also showed that switching to sorafenib after TACE failure/refractoriness resulted in better outcomes than not switching to sorafenib [145].

Should We Wait Until TACE Failure/Refractoriness before Switching to MTAs?

As mentioned above, there is now some degree of global consensus that once TACE failure/refractoriness occurs, patients should be immediately switched to MTAs. However, a detailed review of the 3 major publications to date shows that $20-26 \%$ of patients who were considered to have progressed to TACE failure/refractoriness had already progressed to Child-Pugh class $\mathrm{B}$ or $\mathrm{C}$ $[137,144,145]$. This indicates that if patients continue TACE until they meet the TACE failure/refractoriness criteria, a substantial proportion of patients will not be eligible for receiving MTA therapies because of impaired
Table 9. Criteria of TACE-impossible

1 Impossible to place the catheter selectively due to devastated feeding artery caused by repeated TACE

2 Deterioration of liver function to Child-Pugh $\mathrm{C}$ due to repeated TACE

3 Patients with major vascular invasion $\left(\mathrm{Vp}_{3}\right.$ and $\left.\mathrm{Vp}_{4}\right)$

4 Patients with huge A-P shunting

TACE, transarterial chemoembolization; $\mathrm{Vp}_{3}$, portal vein invasion at the first branch of portal vein; $\mathrm{Vp}_{4}$, portal vein invasion at the main portal vein trunk; A-P shunting, arterial-portal shunting.

liver function. Thus, increasing evidence suggests that switching to molecular targeted therapy after meeting the TACE failure/refractoriness criteria is too late and, thus, this switch should be made at an earlier timing.

Treatment Strategy for TACE Failure/Refractoriness

In addition to MTAs, HAIC is another treatment option for TACE failure/refractoriness, although it generally leads to poor outcomes in patients who have undergone repeated sessions of TACE [146]. Therefore, MTAs should be the first-line treatment in cases of TACE failure/refractoriness. However, MTAs are indicated for patients classified as Child-Pugh class A and thus are not recommended for patients with Child-Pugh class B or worse liver function at the time of TACE failure/refractoriness. HAIC may be an option even in patients with Child-Pugh B liver function depending on individual circumstances (Fig. 3).

\section{Concept of TACE-Impossible}

Apart from TACE failure/refractoriness, it is also important to define the term "TACE-impossible." Patients are considered TACE-impossible upon disappearance/ devastation of the feeding artery due to repeated TACE and/or the development of a parasitic feeding artery, which preclude selective catheterization. Patients whose liver function has worsened to Child-Pugh class $\mathrm{C}$ after repeated TACE are also considered TACE-impossible. However, Child-Pugh class $\mathrm{C}$ patients who undergo superselective TACE, which does not cause liver function deterioration, show an improved prognosis [116-121]. Patients with large A-P shunts or major vascular invasion such as $\mathrm{Vp}_{3}$ or $\mathrm{Vp}_{4}$ disease are also considered TACEimpossible because of the risk of liver failure caused by TACE (Table 9). 
Treatment Strategy for TACE-Impossible Cases

In TACE-impossible cases related to the disappearance/devastation of the feeding artery, transarterial therapy cannot be performed; therefore, MTAs should be used if liver function is Child-Pugh class A. In Child-Pugh class C cases, BSC is usually recommended; however, depending on the patient's condition, superselective TACE may be performed with extreme caution. Liver functional reserve needs to be monitored carefully if embolization is performed in a very limited area [116-121]. MTAs should be considered for patients with large A-P shunts. Patients with $\mathrm{Vp}_{3}$ and $\mathrm{Vp}_{4}$ lesions should be treated with HAIC or MTAs depending on their condition or if they are secondary to TACE failure/refractoriness [147].

\section{Concept of TACE Unsuitability/Ineligibility and Treatment Strategy \\ Consensus Statements}

72. TACE unsuitability includes following 3 conditions. 1. Likely to develop TACE failure/refractoriness

2. Likely to progress to Child-Pugh class B liver function after TACE

3. Unlikely to respond to TACE.

73. Patients classified as TACE-unsuitable/ineligible should be treated with MTAs having high ORR as first line therapy instead of receiving TACE because repeated TACE deteriorates liver function and patients may become ineligible for MTAs as subsequent therapy.

One combination ICI and five MTAs are currently available: atezolizumab plus bevacizumab as first-line therapy, lenvatinib and sorafenib as second-line therapies, and regorafenib, ramucirumab, and cabozantinib as third-line therapies. All 6 regimens demonstrated a survival benefit in clinical trials in patients with advanced HCC (BCLC stage C) or intermediate-stage HCC (BCLC stage $B)$ that progressed on TACE or were ineligible for TACE. Most trials included approximately $10-20 \%$ of patients with intermediate-stage HCC, although the percentage varies among studies.

In the current era of multiple MTAs and combination immunotherapy, the most important issue is establishing therapeutic strategies for patients with intermediatestage HCC, which accounts for the largest proportion of all HCC patients, especially for those unlikely to respond to TACE. Introducing systemic therapy for the first time at an advanced stage of HCC is often too late. Introducing a series of drugs in patients with intermediate-stage HCC who are refractory or unlikely to respond to TACE (TACE-unsuitable) with good liver function may contribute to prolonged survival.

JSH Consensus Statements and Recommendations on HCC
TACE-unsuitable/ineligible patients may also benefit from the following therapeutic strategies: pretreatment with lenvatinib followed by additional selective TACE $[148,149]$. Understanding the concept of "TACE unsuitability/ineligibility" and establishing new strategies for treating these TACE-unsuitable patients are major issues to be addressed in this era of multiple available systemic therapeutic agents [150] including combination immunotherapy.

Heterogeneity of Intermediate-Stage HCC and Treatment Strategies

Intermediate-stage HCC constitutes an extremely heterogeneous population in terms of (1) liver function, (2) tumor size, and (3) number of tumors [151]. In this population, liver function ranges widely from ChildPugh class A (score 5) to class B (score 9), tumor size ranges widely from $<1$ to $>10 \mathrm{~cm}$, and the number of tumors varies considerably from 4 to dozens of nodules, making this an extremely heterogeneous disease.

The current global guidelines recommend TACE as the only standard of care; however, because as many as 6 systemic therapeutic regimens are currently available, it is inaccurate to consider TACE as the only standard of care for intermediate-stage HCC. The evidence supporting TACE was established when MTAs were not available and was based on a comparative trial of TACE with BSC $[152,153]$. In a review article in Gastroenterology, Dr. Bruix, an advocate of the BCLC staging system, states that "Not all patients with intermediate-stage HCC can be considered for TACE. The best candidates for TACE are asymptomatic patients with solitary or limited multifocal HCC with well-preserved liver function (Child-Pugh A or B 7 points without ascites)" [154]. In other words, the development of treatment strategies for patients with bilobar multifocal nodules or large HCCs for which TACE is not indicated or may even worsen the prognosis represents an unmet need.

Various subclassifications and corresponding treatment strategies have been proposed for intermediatestage HCCs [104, 151, 155, 156]. For the subclassification of intermediate-stage HCC, the up-to-7 criteria, which was originally developed to guide transplantation [157], have been widely used worldwide to denote the extent of tumor burden.

Concept of TACE Unsuitability/Ineligibility in Intermediate-Stage HCC

The concept of TACE unsuitability was recently proposed $[148,150,155]$. TACE unsuitability/ineligibility 
Table 10. Criteria and classification of TACE-unsuitable

TACE-unsuitability is defined as each one of the following 3 clinical conditions that prevent a survival benefit from TACE or conditions that TACE is even harmful:

1 Likely to develop TACE failure/refractoriness:

Up-to-7 criteria out nodules

2 Likely to become Child-Pugh B liver function after TACE:

Up-to-7 criteria out nodules (especially, bilobar multifocal nodules), mALBI grade $2 b$

3 Unlikely to respond to TACE:

Confluent multinodular type, massive or infiltrative type, simple nodular type with extranodular growth, poorly differentiated type, intrahepatic multiple disseminated nodules, or sarcomatous changes after TACE

TACE, transarterial chemoembolization; mALBI, modified albumin-bilirubin.

generally refers to the following 3 conditions: (1) likely to develop TACE failure/refractoriness, (2) likely to become Child-Pugh class B liver function after TACE, and (3) unlikely to respond to TACE.

Patients likely to develop TACE failure/refractoriness include those who do not meet the up-to-7 criteria in relation to tumor size and number of tumors [158161]. Patients prone to reduced liver function include those classified as ALBI grade 2 as well as those who do not meet the up-to-7 criteria (especially bilobar multifocal nodules) $[159,162]$. Repeated TACE sessions lower the ALBI score [163], and even a single TACE session in a patient with ALBI grade 2 liver function (especially mALBI grade 2b) [92] may further reduce liver function, which makes the patient ineligible for systemic therapy. Patients with any of the above 3 conditions are unlikely to benefit from TACE in terms of survival and are highly likely to eventually become TACE failure/refractory or Child-Pugh class B. These patients become ineligible for systemic therapy with likely shortened OS. Therefore, TACE is harmful for patients with these conditions. Conditions unlikely to respond to TACE include HCCs with unencapsulated tumors and high incidence of microvascular invasion, such as the simple nodular type with extranodular growth, confluent multinodular type, massive type, infiltrative type, and diffuse type [164], in addition to poorly differentiated HCCs [165] (Table 10).

Outcomes of Treatment with Lenvatinib-TACE

Sequential Therapy in TACE-Unsuitable Patients

Lenvatinib prior to TACE may provide a better survival benefit for TACE-unsuitable patients, such as those with bilobar multifocal nodules, than TACE without prior lenvatinib [155]. A recent proof-of-concept study showed that pretreatment with lenvatinib followed by TACE in patients who did not meet the up-to-7 criteria and were TACE-naive resulted in favorable outcomes [149]. More specifically, the study compared the treatment outcomes of 37 patients who did not meet the upto-7 criteria, were classified as Child-Pugh class A, and received pretreatment with lenvatinib without prior TACE for intermediate-stage HCC to those of 139 patients who did not meet the up-to-7 criteria and received TACE during the same period.

Investigators compared the efficacy of treatment between 30 patients pretreated with lenvatinib (excluding 7 patients who were followed up for $<6$ months) and 60 propensity score-matched patients treated with TACE alone. TACE caused irreversible impairment in liver function compared with lenvatinib, as determined by the ALBI score. Lenvatinib was associated with significantly longer PFS compared with TACE alone (16.0 vs. 3.0 months; HR: 0.19; 95\% CI: 0.10-0.35; $p<0.001)$. Similar results were obtained for OS: 37.9 months with lenvatinib followed by TACE and 21.3 months with TACE alone (HR: 0.48 ; 95\% CI: 0.16-0.79; $p<0.01$ ), demonstrating a clear advantage of the former over the latter. Approximately $70 \%$ of patients pretreated with lenvatinib later received TACE, and 4 achieved CR and were cancer-free and drug-free (including one who became drug-free with lenvatinib alone). Thus, lenvatinib followed by TACE was highly effective in patients who did not meet the up-to-7 criteria and could not be managed with TACE. These results suggest that lenvatinib, which is associated with exceptionally high response rates [155], should be used as 
Fig. 4. Effect of anti-VEGF on tumor vessels. VEGF, vascular endothelial growth factor; TACE, transarterial chemoembolization. Modified with permission from the Japan Society of Hepatology [17].
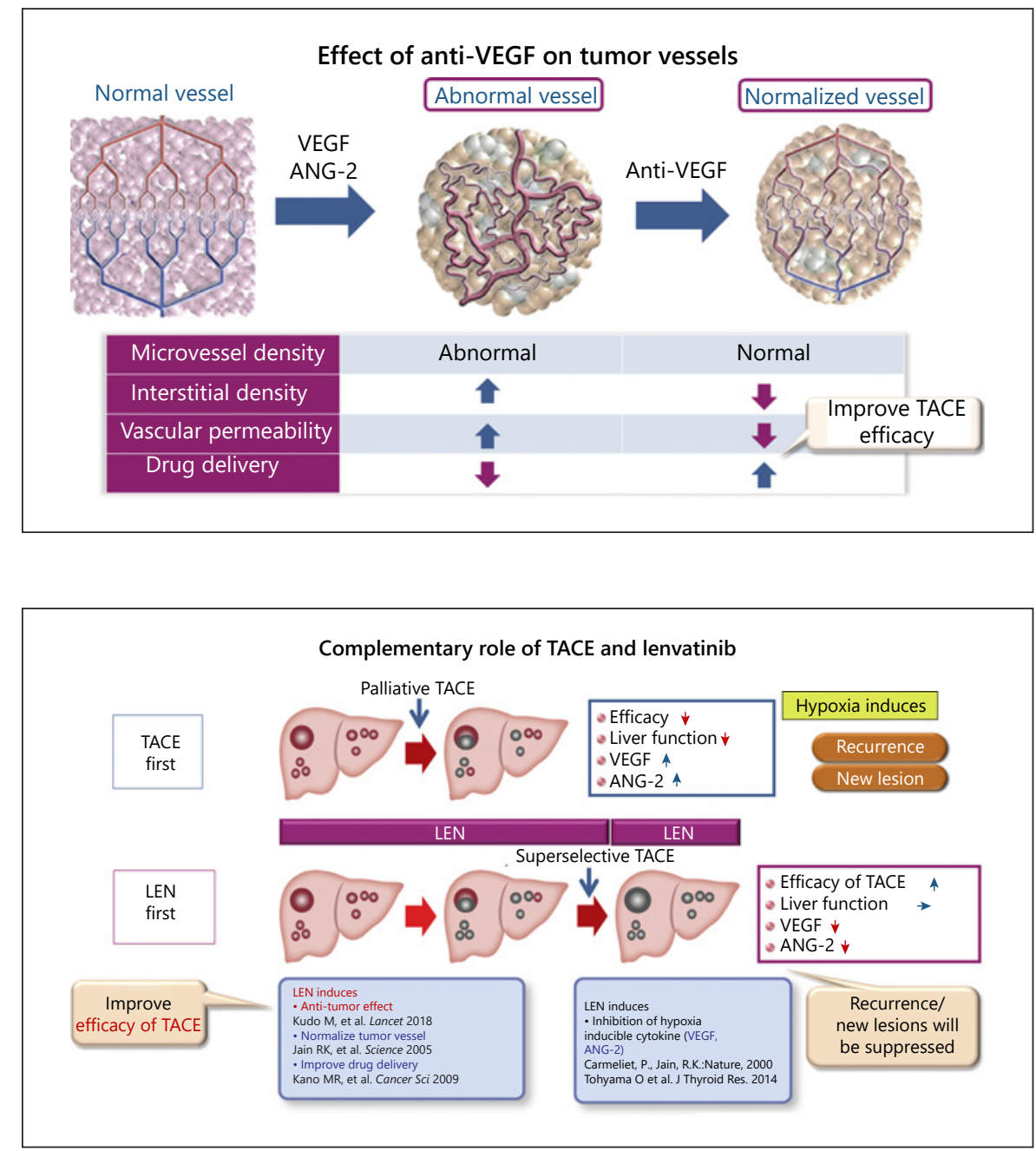

Fig. 5. Complementary role of TACE and lenvatinib. TACE, transarterial chemoembolization; VEGF, vascular endothelial growth factor. Reproduced with permission from the Japan Society of Hepatology [17]. first-line treatment for patients with intermediate-stage HCC with a high tumor burden.

Factors contributing to the favorable OS results in the lenvatinib group include the higher response rate and higher preservation of liver function than TACE. The response rate with lenvatinib was $40.6 \%$ in the REFLECT trial [139], and a higher response rate was reported in Japanese patients with intermediate-stage HCC (61.3\%) [140] and an even higher overall response rate (ORR) in the proof-of-concept study (73.3\%) [149]. These high response rates may be attributed to the fact that many TACE-naive patients have ALBI grade 1 liver function and therefore lower rates of AEs, dose reduction, and treatment interruption/discontinuation, thus allowing for the effective administration of lenvatinib [141].

The high response rate is associated with the following: First, pretreatment with lenvatinib induces tumor regression and necrosis. Second, after pretreatment with lenva-

JSH Consensus Statements and Recommendations on HCC tinib, curative selective TACE can be performed in many cases, resulting in preserved liver function. Third, pretreatment with lenvatinib suppresses hypoxia-induced release of VEGF and other cytokines, thereby preventing recurrence or metastasis $[127,166,167]$. Last, normalization of the tumor vasculature with lenvatinib reduces the permeability of blood vessels and the intratumoral interstitial pressure, which improves the delivery of Lipiodol ${ }^{\circledR}$. containing anticancer drugs throughout the tumor; this increases the embolization effect and results in complete cure (Fig. 4,5). Therefore, lenvatinib-TACE sequential therapy is theoretically effective for patients with intermediate-stage HCC who do not meet the up-to-7 criteria, and it can be used as a standard treatment for intermediate-stage HCC with a high tumor burden (Fig. 6). In addition, there are almost no disadvantages associated with administering lenvatinib prior to TACE in patients with HCC with high tumor burden. Therefore, this therapeu- 
Fig. 6. Changing paradigm of treatment strategy in TACE-unsuitable intermediatestage HCC. HCC, hepatocellular carcinoma; TACE, transarterial chemoembolization. Reproduced with permission from the Japan Society of Hepatology [17].
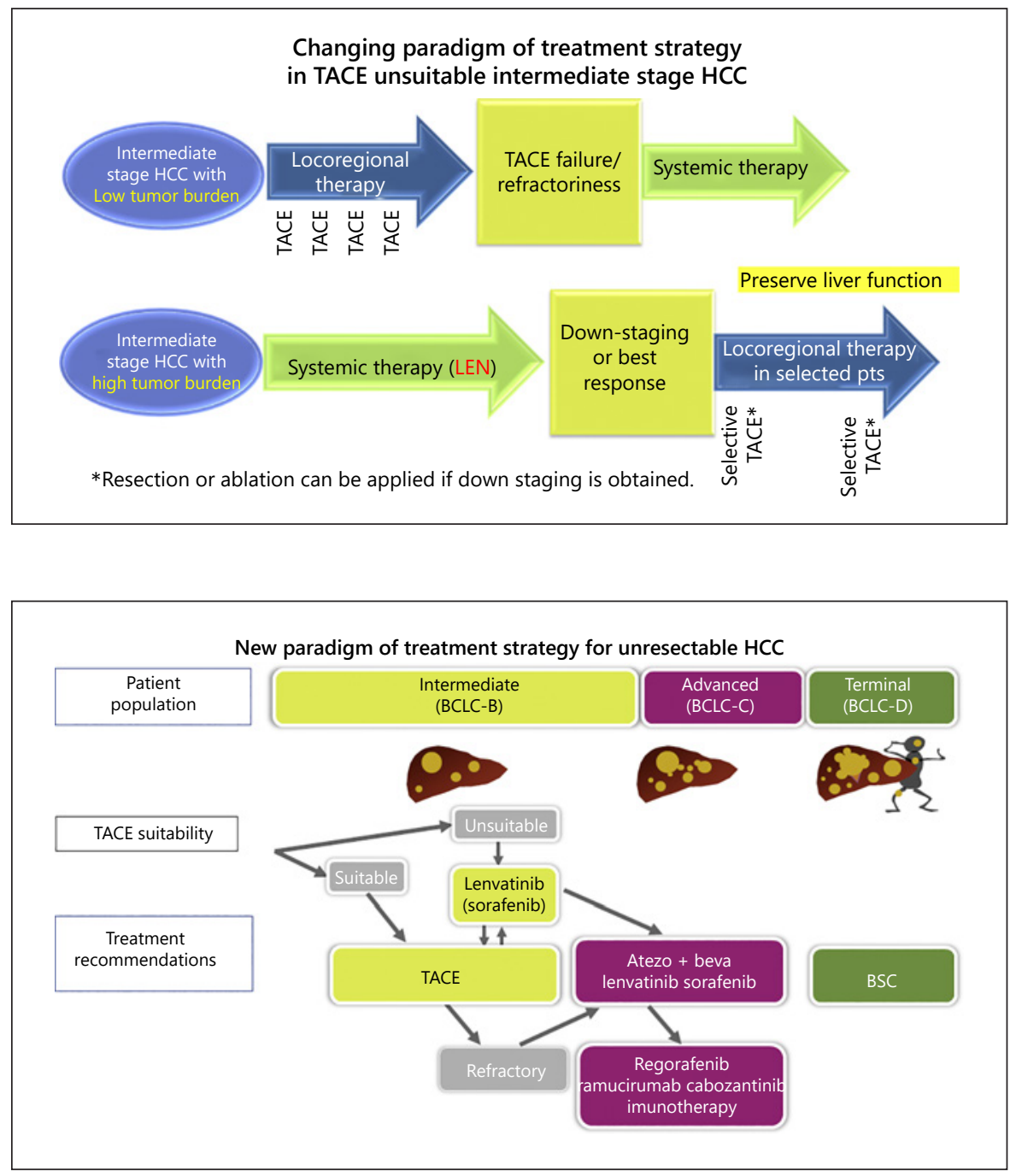

Fig. 7. New paradigm of treatment strategy for unresectable HCC. HCC, hepatocellular carcinoma; TACE, transarterial chemoembolization; BCLC, Barcelona Clinic Liver Cancer. Reproduced with permission from the Japan Society of Hepatology [17]. tic strategy represents a dramatic paradigm shift in the treatment of HCC (Fig. 7). The Asia-Pacific Primary Liver Cancer Expert Consensus Statement includes a similar statement [150].

Patients with bilobar multiple nodules or large HCCs can be pretreated with lenvatinib to normalize the tumor vasculature [127], prevent a surge in VEGF levels after TACE, and reduce the residual tumor burden before selective TACE to ensure favorable treatment outcomes and preserve liver functional reserve (Fig. 8). This may also be one of the reasons why, among all clinical trials of TACE plus MTAs, the TACTICS study, in which patients were pretreated with the MTA sorafenib, was the only successful trial [130-135] (Table 7).

Taken together, the current findings indicate that lenvatinib is a rational treatment for TACE-unsuitable patients, such as those who do not meet the up-to-7 criteria.
It can be considered as a first-line treatment for conditions such as intermediate-stage HCC with high tumor burden or TACE-resistant HCC, and for patients with ALBI grade 2 (or mALBI grade $2 b$ ) liver function. Furthermore, lenvatinib is associated with high response rates in poorly differentiated HCC [168-170], and it is effective against HCCs of the massive nodular type, diffuse/infiltrative type, confluent multinodular type, and simple nodular type with extranodular growth. Thus, lenvatinib-TACE sequential therapy can be a rational and effective treatment not only for patients who do not meet the up-to-7 criteria but also for those with TACE-resistant HCC and those prone to reduced liver functional reserve, such as those classified as ALBI grade 2b (Fig. 7; Table 10).

There are 3 types of HCCs for which TACE is unsuitable or ineligible (i.e., TACE is not beneficial or is even 


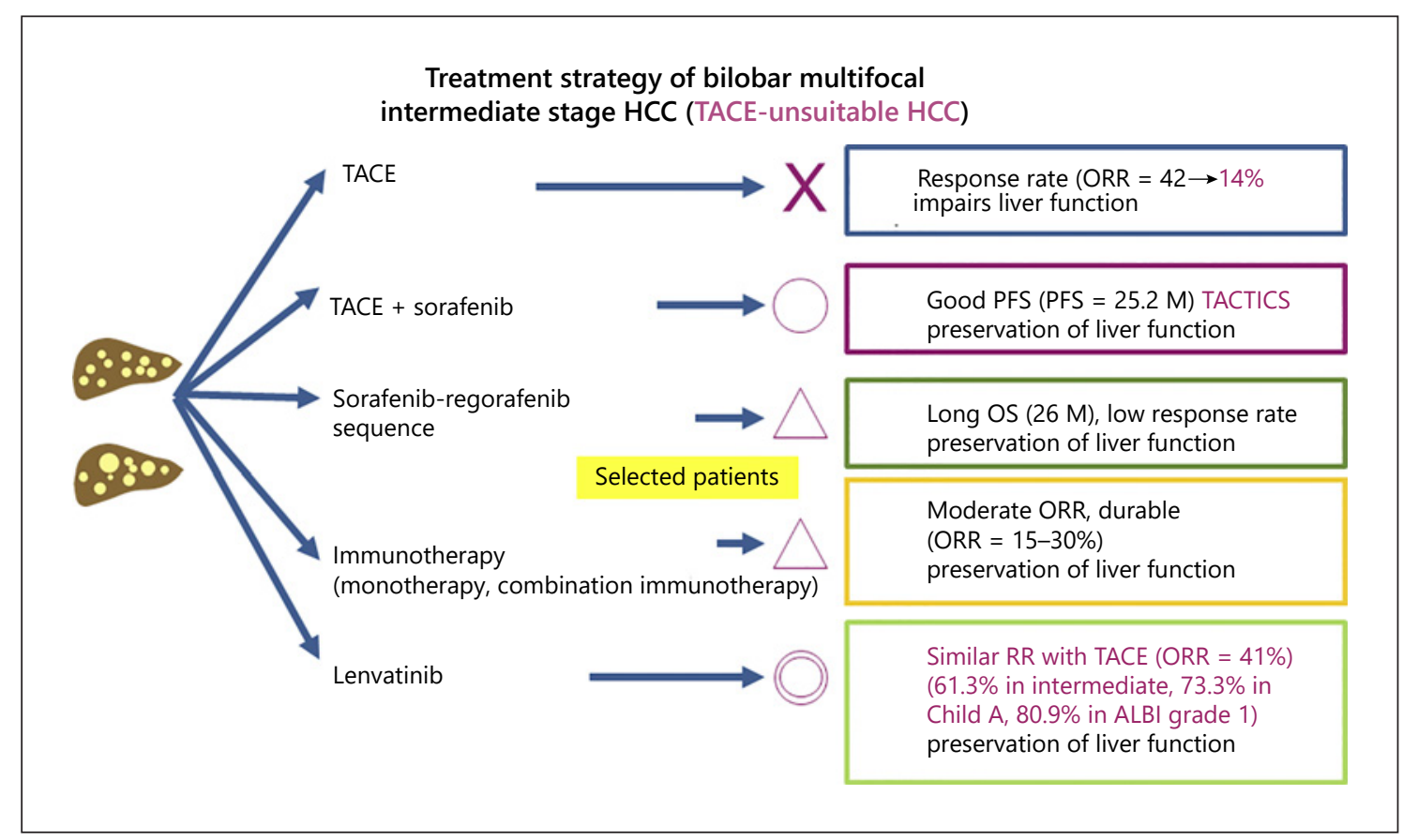

Fig. 8. Treatment strategy for bilobar multifocal intermediate-stage HCC (TACE-unsuitable HCC). HCC, hepatocellular carcinoma; TACE, transarterial chemoembolization. Reproduced with permission from the Japan Society of Hepatology [17].

harmful): (1) lesions that are likely to become TACE refractory after TACE, (2) lesions that predispose patients to deteriorate to Child-Pugh class B liver function, and (3) lesions unlikely to respond to TACE. For patients with these types of intermediate-stage HCC, lenvatinib followed by additional selective TACE will improve the efficacy of TACE and prolong survival $[148,149]$. Therefore, lenvatinib and TACE are complementary: pretreatment with lenvatinib enhances the efficacy of TACE, and this does not occur in a competitive manner; both treatments are necessary. A complete understanding of the roles of each treatment modality is necessary for treatment planning in intermediate-stage HCC.

Because more drugs are being developed, a shift to a new treatment paradigm is highly likely. Systemic drug therapy will be the first choice for patients with intermediate-stage HCC that is not curable with TACE, followed by selective TACE (or even resection or ablation) on an on-demand basis (Fig. 6, 7). Clinical studies are being planned to compare TACE with not only lenvatinib but also with combination immunotherapies, and the results are eagerly awaited (Fig. 10).

\section{Hepatic Arterial Infusion Chemotherapy}

\section{Consensus Statements}

74. HAIC is indicated in patients with major portal vascular invasion and in those with intrahepatic multinodular lesions having Child-Pugh class B liver function.

75. Systemic therapy is recommended in patients with bilobar multifocal HCCs and Child-Pugh A liver function.

Concept and Indications for Treatment

In Japan, HAIC has conventionally been used for treating intrahepatic advanced HCC. However, the recent introduction of multiple systemic therapies for the treatment of HCC has decreased the use of HAIC, and there are no studies comparing HAIC with systemic therapies. In clinical practice, HAIC is used in patients with tumor invasion to the main portal vein, in patients with impaired liver function who are not eligible for systemic therapy, and in those who progress to advanced disease after systemic therapy. The 3 most common HAIC regimens used in Japan are (1) low-dose 5-FU plus CDDP (low-dose FP), (2) HAIC with interferon, and (3) HAIC with CDDP. 
Low-dose FP is based on the concept of biochemical modulators, in which a modulator anticancer drug (CDDP) is administered before an effector anticancer drug (5-FU) to alter the pharmacokinetics of the effector drug, increase its antitumor effect, and reduce its toxicity to normal cells, thereby increasing the efficacy of chemotherapy. Low-dose FP is indicated for patients with multiple intrahepatic nodules in patients with Child-Pugh B liver function or patients with portal vein tumor thrombosis (PVTT) who are ineligible for or unresponsive to hepatectomy, RFA, TACE, and systemic therapy. In patients with extrahepatic spread, this therapy is indicated if the intrahepatic lesion has progressed and is considered a prognostic factor because small distant metastases usually do not affect prognosis.

HAIC plus Targeted Therapy

\section{Consensus Statement}

76. The combination of HAIC and the MTA such as sorafenib is expected to provide a survival benefit in patients with major vascular invasion.

HAIC has the advantage of increasing the local concentration of anticancer drugs in the tumor area. This is achieved by local drug administration through the hepatic artery, which minimizes the systemic adverse effect of the drugs. HAIC was developed in Japan and has been widely used as a chemotherapeutic option for HCC. This therapy is highly effective in terms of local control and has been used as an effective treatment for advanced HCC with major vascular invasion. However, there are no RCTs showing a survival benefit associated with HAIC.

Rationale for Combining MTAs with HAIC

HAIC is effective for local control, although there is a lack of evidence supporting its survival benefit. By contrast, sorafenib has shown a survival benefit but has a poor local control effect (i.e., antitumor effect) [171]. Because of the overlap in the target disease stages of HAIC and MTAs, the selection between HAIC and sorafenib remains controversial. However, combining the 2 may confer the benefits of both the local control effect of HAIC and the survival benefit of MTAs. The use of MTAs is expected to improve tumor vascular permeability and tumor interstitial pressure, resulting in improved distribution of the infused chemotherapeutic agents and enhanced efficacy of HAIC.
Clinical Trials of Combination Therapy with MTAs and HAIC

The phase 2 study of sorafenib plus cisplatin HAIC [172] was conducted as a randomized trial comparing sorafenib alone with the combination of sorafenib and one-shot cisplatin arterial infusion. The primary endpoint of OS was 8.7 months in the sorafenib-alone group and 10.6 months in the sorafenib plus HAIC group (HR: 0.60 ; $95 \%$ CI: $0.38-0.96 ; p=0.031$ ), suggesting the efficacy of combination therapy (Table 11).

In a randomized phase 3 trial comparing sorafenib monotherapy with low-dose cisplatin combined with fluorouracil HAIC (low-dose FP) (SILIUS study) [173], investigators evaluated whether low-dose FP would provide an additional benefit in terms of OS. The primary endpoint of OS was 11.5 months in the sorafenib-alone group versus 11.8 months in the combination group (HR: 1.009; 95\% CI: 0.743-1.371; $p=0.955$, log-rank). The difference was not statistically significant, and the trial failed to demonstrate an additional benefit of low-dose FP to sorafenib. However, a sub-analysis in this study showed that the OS in $\mathrm{Vp}_{4}$ cases was 6.5 months in the sorafenibalone group versus 11.4 months in the combination group (HR: 0.493; 95\% CI: 0.240-1.014; $p=0.050$, log-rank), demonstrating a marginally significant additional benefit. The response rate was $17.5 \%$ in the sorafenib-alone group, whereas it was significantly higher in the combination group at $36.3 \%(p=0.003)$. Also, in the combination group, OS was significantly longer in responders (CR + $\mathrm{PR})$ at 23.0 months than in nonresponders at 9.9 months $(\mathrm{SD}+\mathrm{PD})(p<0.001)$ (Table 11).

A randomized phase 3 trial comparing sorafenib monotherapy with sorafenib plus HAIC (FOLFOX regimen) [174] was conducted in patients with advanced HCC with portal vein tumor thrombosis. The primary endpoint of OS was 7.13 months in the sorafenib-alone group and 13.37 months in the sorafenib plus FOLFOX HAIC group (HR: 0.35; 95\% CI: $0.26-0.48 ; p=0.001$ ), demonstrating a significant prolongation of survival with concomitant use of HAIC. The response rate was significantly higher in the combination group than in the sorafenib-alone group (3 vs. $51 \%$, respectively, $p<0.001$ ).

\section{Propensity Score-Matched Comparisons of MTAs} and HAIC

The results of a large retrospective cohort study of 2006 patients with advanced HCC (1,465 treated with sorafenib and 541 with HAIC) were recently reported [175]. Among patients with no extrahepatic spread but with vascular invasion, OS was significant longer in the 
Table 11. Clinical trials of HAIC + sorafenib versus sorafenib alone

\begin{tabular}{|c|c|c|c|c|c|c|}
\hline & \multicolumn{2}{|l|}{ Ikeda et al. [172] } & \multicolumn{2}{|l|}{ Kudo [173] } & \multicolumn{2}{|l|}{ He et al. [174] } \\
\hline Trial name & \multicolumn{2}{|l|}{ HCC_Sor_CDDP_rP2 } & \multicolumn{2}{|l|}{ SILIUS } & \multicolumn{2}{|l|}{ na } \\
\hline Phase & \multicolumn{2}{|l|}{ Phase 2} & \multicolumn{2}{|l|}{ Phase 3} & \multicolumn{2}{|l|}{ Phase 3} \\
\hline Design & \multicolumn{2}{|l|}{ RCT } & \multicolumn{2}{|l|}{ RCT } & \multicolumn{2}{|l|}{ RCT } \\
\hline \multirow[t]{2}{*}{ Patients } & \multicolumn{2}{|l|}{ Child-Pugh class A } & \multicolumn{2}{|c|}{ Child-Pugh class A/B7 } & \multicolumn{2}{|l|}{ Child-Pugh class A } \\
\hline & \multicolumn{2}{|l|}{ BCLC stage $\mathrm{B} / \mathrm{C}$} & \multicolumn{2}{|l|}{ BCLC stage $\mathrm{B} / \mathrm{C}$} & \multicolumn{2}{|c|}{ BCLC stage $\mathrm{C}$ with $\mathrm{Vp}$} \\
\hline Comparator & \multicolumn{2}{|l|}{ Sorafenib } & \multicolumn{2}{|l|}{ Sorafenib } & \multicolumn{2}{|l|}{ Sorafenib } \\
\hline HAIC regimen & \multicolumn{2}{|l|}{ CDDP } & \multicolumn{2}{|c|}{ CDDP/5FU (low-dose FP) } & \multicolumn{2}{|c|}{ Oxaliplatin/leucovorin/5FU (FOLFOX) } \\
\hline \multirow[t]{2}{*}{ Patients, $n$} & \multicolumn{2}{|l|}{108} & \multicolumn{2}{|l|}{206} & \multicolumn{2}{|l|}{247} \\
\hline & Sorafenib + HAIC & Sorafenib & Sorafenib + HAIC & Sorafenib & Sorafenib + HAIC & Sorafenib \\
\hline OS, months & 10.6 & 8.7 & 11.8 & 11.5 & 13.37 & 7.13 \\
\hline HR $(95 \% \mathrm{CI}), p$ value & \multicolumn{2}{|l|}{$0.60(0.38-0.96), 0.031$} & \multicolumn{2}{|c|}{$1.009(0.743-1.371), 0.955$} & \multicolumn{2}{|c|}{$0.35(0.26-0.48),<0.001$} \\
\hline
\end{tabular}

HAIC, hepatic arterial infusion chemotherapy; RCT, randomized controlled trial; BCLC, Barcelona Clinic Liver Cancer; OS, overall survival; HR, hazard ratio; $\mathrm{CI}$, confidence interval.

Table 12. Overall survival according to absence or presence of $\mathrm{Vp}$ : combination of sorafenib plus HAIC versus sorafenib alone

\begin{tabular}{|c|c|c|c|c|}
\hline & $\mathrm{Vp}_{0}$ & $\mathrm{Vp}_{1}$ & $\mathrm{Vp}_{3}$ & $\mathrm{Vp}_{4}$ \\
\hline $\begin{array}{l}\text { Ikeda et al. } \\
\text { Annals of } \\
\text { Oncology } 2016 \\
{[172]}\end{array}$ & $\begin{array}{c}12.9 \mathrm{M} \text { versus } 11.6 \mathrm{M} \\
\text { HR } 0.646(0.342-1.220) \\
p=0.173\end{array}$ & \multicolumn{3}{|c|}{$\begin{array}{c}9.1 \mathrm{M} \text { versus } 7.1 \mathrm{M} \\
\mathrm{HR}=0.579(0.308-1.090)(\mathrm{Vp} 1-\mathrm{Vp} 4) \\
p=0.086\end{array}$} \\
\hline $\begin{array}{l}\text { Kudo et al. } \\
\text { Lancet GH } 2018 \\
{[173]}\end{array}$ & $\begin{array}{c}11.3 \mathrm{M} \text { versus } 11.9 \mathrm{M} \\
\text { HR } 1.001(0.623-1.608) \\
p=0.996\end{array}$ & \multicolumn{2}{|c|}{$\begin{array}{c}12.6 \mathrm{M} \text { vs. } 14.4 \mathrm{M} \\
\text { HR } 1.367(0.829-2.255)(\mathrm{Vp} 1-\mathrm{Vp} 3) \\
p=0.423\end{array}$} & $\begin{array}{c}11.4 \mathrm{M} \text { vs. } 6.5 \mathrm{M} \\
\text { HR } 0.493(0.240-1.014)(\mathrm{Vp} 4) \\
p=0.050\end{array}$ \\
\hline $\begin{array}{l}\text { He et al. } \\
\text { JAMA Oncology } \\
2019[174]\end{array}$ & & $\begin{array}{c}18.17 \mathrm{M} \text { vs. } 10.87 \mathrm{M} \\
0.33(0.16-0.68)(\mathrm{Vp} 1-\mathrm{Vp} 2) \\
p=0.002\end{array}$ & $\begin{array}{c}13.47 \mathrm{M} \text { vs. } 6.27 \mathrm{M} \\
0.29(0.18-0.47)(\mathrm{Vp} 3) \\
p<0.001\end{array}$ & $\begin{array}{c}9.47 \mathrm{M} \text { vs. } 5.5 \mathrm{M} \\
0.40(0.21-0.77)(\mathrm{Vp} 4) \\
p<0.001\end{array}$ \\
\hline
\end{tabular}

Vp, portal vein invasion; HAIC, hepatic arterial infusion chemotherapy; HR, hazard ratio.

HAIC group than in the sorafenib group (10.6 vs. 9.1 months, respectively; HR: 0.667; 95\% CI: 0.475-0.935; $p=0.018$ ). By contrast, among patients with no distant metastasis or vascular invasion, there was no significant difference in OS between the HAIC and sorafenib groups. This was an unprecedented large-scale cohort study, and the results suggest that HAIC is effective in patients with major vascular invasion but not in patients without major vascular invasion (Table 12).

The efficacy of HAIC as posttreatment after sorafenib has been suggested [176]; however, this was reported in a retrospective study without sufficient levels of evidence. Therefore, at present, the selection of second-line treatment after first-line molecular targeted therapy should be made on a case-by-case basis.

\section{Systemic Therapy}

\section{Consensus Statements}

77. Atezolizumab in combination with bevacizumab is recommended as first-line treatment for unresectable advanced HCCs that are not amenable to surgical resection, liver transplantation, locoregional 
therapy, or TACE in patients with good PS and good liver function classified as Child-Pugh grade A.

78. Second-line treatment with sorafenib or lenvatinib is recommended for Child-Pugh class A patients who failed on treatment with atezolizumab plus bevacizumab because of radiographic progression or unacceptable AEs.

79. Third-line treatment with regorafenib is recommended for Child-Pugh class A patients who have progressed radiographically on treatment with sorafenib but tolerated sorafenib. Treatment with ramucirumab is also recommended for patients with Child-Pugh class A liver function and serum AFP $\geq 400 \mathrm{ng} / \mathrm{mL}$ who have discontinued treatment with sorafenib or lenvatinib because of radiologic progression or AEs. Treatment with cabozantinib is recommended for patients who progressed on or are intolerant to sorafenib/lenvatinib.

The JSH Clinical Practice Guidelines for HCC 2017 [9] recommend molecular targeted therapy for patients classified as Child-Pugh class A with extrahepatic metastasis, vascular invasion, or $\geq 4$ intrahepatic multiple nodules without vascular invasion or extrahepatic spread. As of December 2020, there are 5 MTAs and 1 combination immunotherapy approved in Japan: atezolizumab plus bevacizumab as first-line treatment, sorafenib and lenvatinib as second-line treatments and regorafenib, cabozantinib and ramucirumab as third-line treatments.

Clinical Trials of MTAs and Recommendation in Guidelines

Sorafenib was first approved in 2007 based on the results of the SHARP [177] and Asia-Pacific [178] trials, and it was the only MTA approved in Japan for a long time since May 2009. Over the next 10 years, many clinical trials were conducted for both first- and second-line treatments, but most trial results were negative. In 2017, the RESORCE trial [179] demonstrated for the first time that regorafenib as a second-line agent was effective for prolonging survival in patients with HCC who had tolerated sorafenib, leading to its approval in Japan. In March 2018, lenvatinib was approved as a first-line treatment based on the results of the REFLECT trial [139], which demonstrated the non-inferiority of lenvatinib in a headto-head comparison with sorafenib.

Subsequently, in June 2019, ramucirumab was approved for the treatment of HCC in patients with an AFP of $\geq 400 \mathrm{ng} / \mathrm{mL}$ based on positive results from the REACH-2 study [180]. In September 2020, atezolizumab plus bevacizumab was approved based on positive results from the IMbrave150 trial, and in November 2020, cabozantinib was approved based on positive results from the CELESTIAL trial [181] and briding phase 2 trial conducted in Japan [182]. Therefore, as of December 2020, approval has been granted to 1 first-line combination immunotherapy (atezolizumab plus bevacizumab), $2 \mathrm{sec}-$ ond-line MTAs (sorafenib and lenvatinib) and 3 third-line agents (regorafenib [only for patients with confirmed tolerance to sorafenib] and ramucirumab [only for those with AFP $\geq 400 \mathrm{ng} / \mathrm{mL}]$ ), which can now be used in daily clinical practice in Japan.

The recommendations of the EASL guidelines [70] and AASLD guidelines [71] for first- and second-line therapies are similar to those of the JSH guidelines. At present, atezolizumab plus bevacizumab combination therapy is commonly used as the first-line treatment in Japan. The next commonly used agent is lenvatinib. Clinical trials have demonstrated the efficacy of second-line therapies only after sorafenib; there are no trials of drugs used after lenvatinib. Nevertheless, lenvatinib is associated with a high response rate and is used not only as a first-line agent but also as a second-line and third-line agent. More specifically, the other 4 MTAs prolong survival by disease stabilization, not by inducing tumor necrosis.

\section{Clinical Trials of Immunotherapies}

ICI Monotherapy. A clinical trial of nivolumab as a single agent for the first-line treatment of advanced HCC (CheckMate 459) and a clinical trial of pembrolizumab as a second-line treatment (KEYNOTE 240) were conducted using placebo as a control. In the CheckMate-040 phase $1 / 2$ study [183], nivolumab demonstrated extremely high efficacy as a second-line treatment after sorafenib, with an OS of 15.6 months, leading to the FDA-granted accelerated approval of nivolumab as a second-line treatment after sorafenib. Pembrolizumab also demonstrated a survival benefit in the phase 1/2 KEYNOTE-224 study [184], and it received accelerated approval from the FDA as a second-line treatment after sorafenib. These ICIs are currently approved in many regions and countries around the world, including North America, Australia, and Taiwan. They are also available for use, although not approved yet, in Europe without insurance coverage. As such, these agents are widely used worldwide except in Japan, where the Ministry of Health, Labour and Welfare has issued a notice prohibiting the off-label use of any drugs at high-level hospitals including university hospitals. Neither of these ICIs, nivolumab and pembrolizum$a b$, is available for HCC treatment, leading to a drug lag 


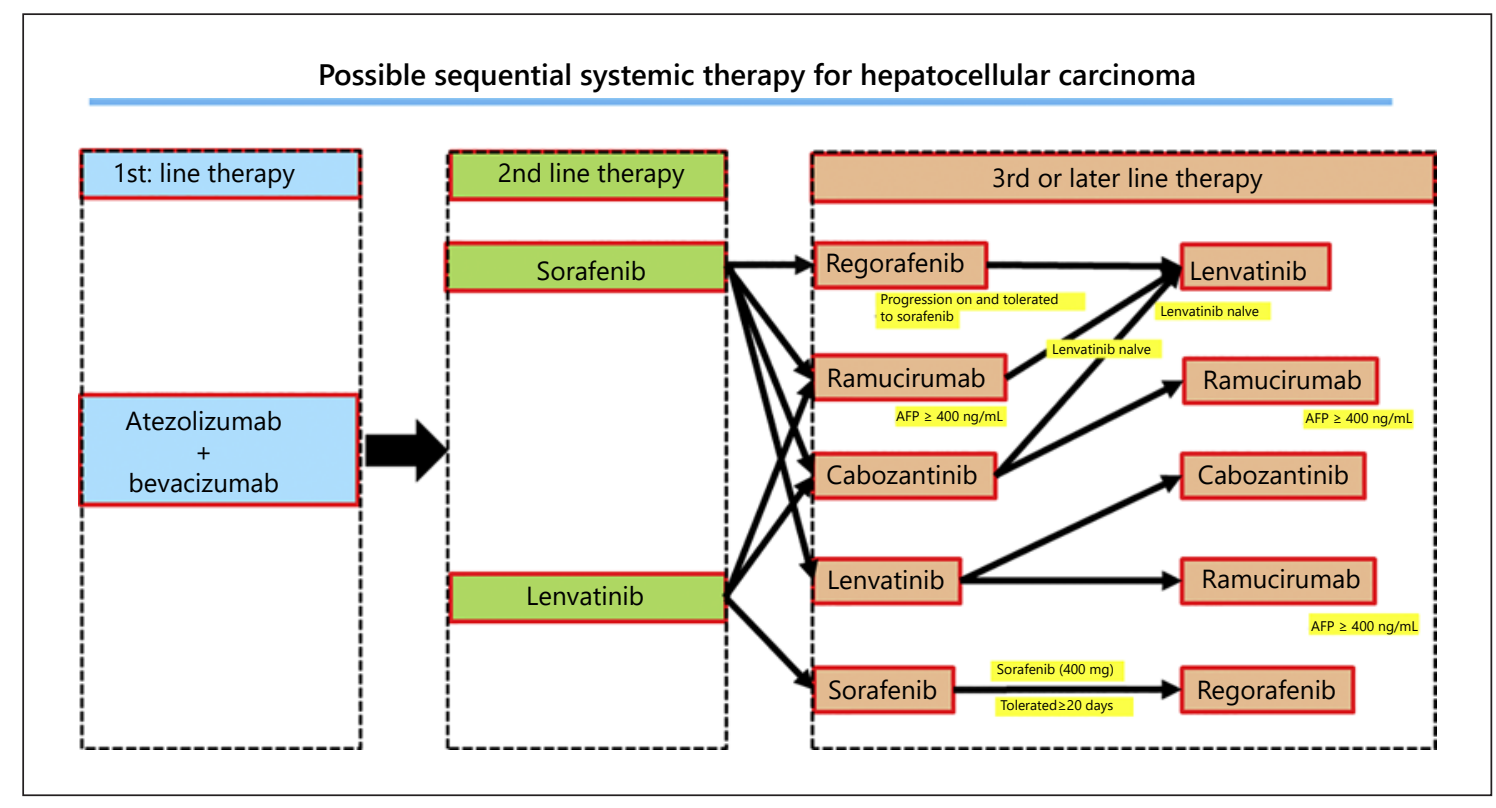

Fig. 9. Possible sequential systemic therapy for HCC. HCC, hepatocellular carcinoma. Reproduced with permission from the Japan Society of Hepatology [17].

status of $>2$ years. Moreover, the KEYNOTE-240 study, the phase 3 trial of pembrolizumab, ended with negative results [185]. The CheckMate 459 study also ended with negative results, which were presented at the ESMO 2019 [186]. However, this drug lag situation was terminated by the approval of atezolizumab plus bevacizumab combination therapy.

Clinical Trials of ICIs in Combination with Anti-

VEGF/TKIs for Advanced HCC

The results of the IMbrave150 study, a phase 3, international, prospective study comparing atezolizumab plus bevacizumab with sorafenib, were presented at the ESMO Asia in November 2019 and published in 2020 [187]. Regarding systemic therapy for HCC, no drugs have ever shown superiority over sorafenib in the 12 -year period since its approval in 2007.

In the first interim analysis of the IMbrave150 study, atezolizumab plus bevacizumab combination was superior to first-line treatment with sorafenib, showing remarkably better HRs for OS (HR $=0.58, p=0.0006)$ and PFS (HR $=0.59, p<0.0001)$, and substantial superiority to sorafenib in terms of ORR, safety, and quality of life [187]. These results are underpinned by the theoretical principle that the anti-VEGF antibody (bevacizumab) changes the immune microenvironment from immunosuppressive to immune-responsive. In that environment,
PD-L1 antibodies can enhance the antitumor activity of immune-exhausted CD8-positive T cells by blocking the PD-1/PD-L1 pathway. Thus, the cancer microenvironment and $T$ cells (and even cancer cells) responded in a manner consistent with the theoretical scenario. The results were certainly reasonable, and they have completely changed the paradigm of clinical practice $[188,189]$. The combination of atezolizumab and bevacizumab has already been approved worldwide. It is thus obvious that currently, first-line treatment with sorafenib and lenvatinib will be positioned as second-line treatment, and regorafenib, ramucirumab, and cabozantinib will be third-line treatment agents (Fig. 9).

Another Combination Immunotherapy with AntiVEGF/TKI for Advanced HCC

Phase $1 \mathrm{~b}$ study of pembrolizumab plus lenvatinib showed favorable results: ORR of $36.0 \%$, PD rate of $7.0 \%$, PFS of 8.6 months, and OS of 22.0 months [190]. Currently, a phase 3 trial (LEAP-002) of this combination is ongoing, and its results are awaited (Fig. 10).

Clinical Trial of PD-1/PD-L1 Antibody in Combination with Anti-CTLA-4 Antibody

Other ongoing clinical trials of ICI-based combination therapies for advanced HCC include a phase 3 trial of the combination of the anti-PD-L1 antibody durvalumab and 


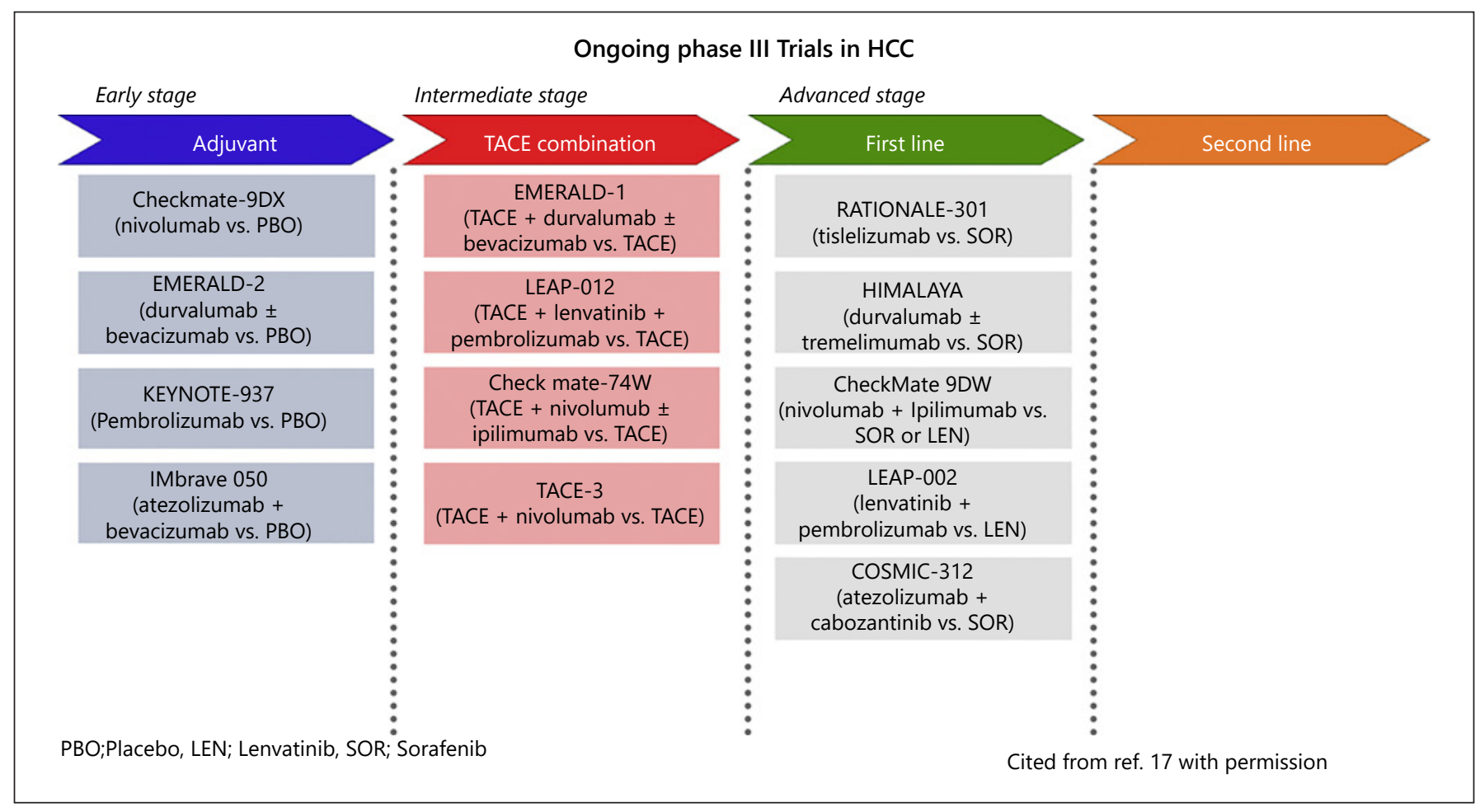

Fig. 10. Ongoing phase 3 trials in HCC. HCC, hepatocellular carcinoma; TACE, transarterial chemoembolization; PBO, placebo; LEN, lenvatinib; SOR, sorafenib. Reproduced with permission from the Japan Society of Hepatology [17].

the anti-CTLA-4 antibody tremelimumab (HIMALAYA study; Fig. 10). Phase 1/2 trial results suggest that this combination is promising: the response rate with the combination treatment was $>2$-fold higher (24\%) than that with single-agent durvalumab (10.6\%) [191]. In addition, OS in the combination arm was longer (18.7 months) than that in the durvalumab monotherapy arm (13.6 months).

The results of a clinical trial of nivolumab and ipilimumab (phase 1/2, cohort 4 of the CheckMate-040 study), which were presented at the ASCO 2019 [192], also showed that the combination of nivolumab $1 \mathrm{mg} / \mathrm{kg}$ and ipilimumab $3 \mathrm{mg} / \mathrm{kg}$ resulted in an extremely good outcome, with a response rate of $32 \%$ (based on independent imaging review according to the RECIST 1.1 criteria) and a median OS of 22.8 months. Based on these promising results, the phase 3 trial (CheckMate-9DW) is ongoing (Fig. 10).

\section{Clinical Trials of the Combination of TACE and Immunotherapy}

The combination of TACE and immunotherapy \pm bevacizumab is also being investigated in the phase $3 \mathrm{EM}$ ERALD-1 study, a 3-arm global clinical trial comparing TACE followed by durvalumab plus bevacizumab, TACE followed by durvalumab, and TACE followed by placebo. The results are awaited (Fig. 10). A phase 3 clinical trial of TACE plus combination treatment with pembrolizumab and lenvatinib (LEAP-012) is also underway (Fig. 10). Another phase 3 trial of nivolumab plus ipilimumab (CheckMate-74W) in combination with TACE is ongoing (Fig. 10) [193].

\section{Clinical Trials in the Adjuvant Setting}

Four phase 3 trials in the adjuvant setting are ongoing, including single-agent nivolumab (CheckMate-9DX) or pembrolizumab (KEYNOTE-937) after hepatectomy or RFA, the EMERALD-2 study ( 3 arms: durvalumab alone, durvalumab plus bevacizumab, and placebo), and the IMbrave050 study of the combination of atezolizumab and bevacizumab compared with placebo (Fig. 10) [193].

\section{How to Select Systemic Therapy or HAIC in Intermediate- and Advanced-Stage HCC}

\section{Consensus Statements}

80. Systemic therapy is preferred for TACE-refractory/ impossible patients with Child-Pugh class A liver 
function who are not amenable to resection or locoregional therapy without vascular invasion.

81. For Child-Pugh class A patients with HCC not amenable to resection or locoregional therapy with vascular invasion, systemic therapy should be considered, but if impaired liver function or rapid tumor growth is anticipated because of major vascular invasion, HAIC should be considered. In patients who develop vascular invasion after repeated TACE and become refractory to TACE, systemic therapy is preferred because these patients are unlikely to respond to HAIC.

According to the JSH Clinical Practice Guidelines for HCC 2017 [9], either HAIC or molecular targeted therapy can be selected for patients with no extrahepatic metastasis who show vascular invasion or $\geq 4$ tumors with Child-Pugh class A liver function. However, a large retrospective multicenter study showed no difference in outcomes between HAIC and sorafenib in patients without vascular invasion [175], suggesting that MTAs are preferred for patients with good PS and no comorbidities that may preclude their introduction. For patients with major vascular invasion, the efficacy of HAIC was suggested in the same study [175], underscoring the importance of distinguishing between patients suitable for HAIC and those suitable for MTAs in clinical practice.

There are 2 possible orders and methods of use for these 2 therapies. The first is to give HAIC first, and then, if the patient does not respond, immediately switch to MTAs. The second is to give MTAs first, and then, if the disease worsens, switch to HAIC. HAIC for patients with vascular invasion is often performed via an implanted port catheter system (reservoir), and it is associated with technical issues related to the implantation and maintenance of the reservoir. Therefore, deciding which therapy to use depends on the situation at each center. Centers with interventional radiology capability tend to opt for HAIC, whereas MTAs can be introduced at any center with experience in managing the AEs of MTAs. Regarding liver function, MTAs are indicated in Child-Pugh class A patients, whereas HAIC is performed even in Child-Pugh class B patients; therefore, patients whose liver functional reserve was impaired by molecular targeted therapy are still eligible for HAIC.

In summary, systemic therapy is the preferred treatment for Child-Pugh class A patients with HCC not amenable to resection or locoregional therapy without vascular invasion, provided that the patient has good PS and no comorbidities that preclude the introduction of these drugs. For Child-Pugh class A patients with HCC not amenable to resection or locoregional therapy who show

JSH Consensus Statements and Recommendations on HCC vascular invasion, molecular targeted therapy may be considered [194]. However, if impaired liver function or rapid tumor growth is anticipated because of the presence of major vascular invasion or elevated tumor markers, HAIC should be considered as the first-line treatment. For those who develop vascular invasion after undergoing repeated sessions of TACE and become refractory to TACE, MTAs are preferred because they may not respond well to HAIC [147].

\section{Transplantation}

Consensus Statements

82. Liver transplantation is an ideal treatment performed with curative intent for both HCC and liver cirrhosis.

83. Most liver transplants in Japan are performed using living donors.

84. Although insurance coverage for living-donor liver transplantation is limited to HCC patients classified as Child-Pugh class $\mathrm{C}$ and who meet the Milan criteria, the eligibility criteria for insurance coverage for liver transplantation have been expanded to include patients meeting the Milan criteria or the 5-5500 criteria ( $\leq 5 \mathrm{~cm}, \leq 5$ tumors, AFP $\leq 500 \mathrm{ng} / \mathrm{mL}$ ) since 2019.

Liver transplantation is an ideal treatment performed with curative intent for both HCC and comorbid liver disease, as it can not only remove the cancer itself but also treat underlying liver abnormalities such as cirrhosis. Therefore, it is clinically significant. There are 2 types of liver transplants, brain-dead liver transplant and livingdonor liver transplant; the former is more common in Western countries and the latter is more common in Japan.

A total of 9,643 liver transplants were performed in Japan by the end of 2018 [195]; the vast majority of these $(9,136,94.7 \%)$ involved living donors. This is in contrast to the West, where liver transplants from brain-dead donors are the norm. Approximately 450 liver transplants are currently performed annually, and after the full enactment of the revised Organ Transplant Act in July 2010, the number of liver transplants from brain-dead donors has been increasing gradually, accounting for up to $15 \%$ of all liver transplants performed in 2018. However, the number remains low compared with that in Western countries and South Korea.

As of the end of 2018, as many as 1,688 living-donor liver transplants had been performed for HCC, accounting for $18.5 \%$ of all living-donor liver transplants; braindead liver transplants for HCC amounted to only 30 


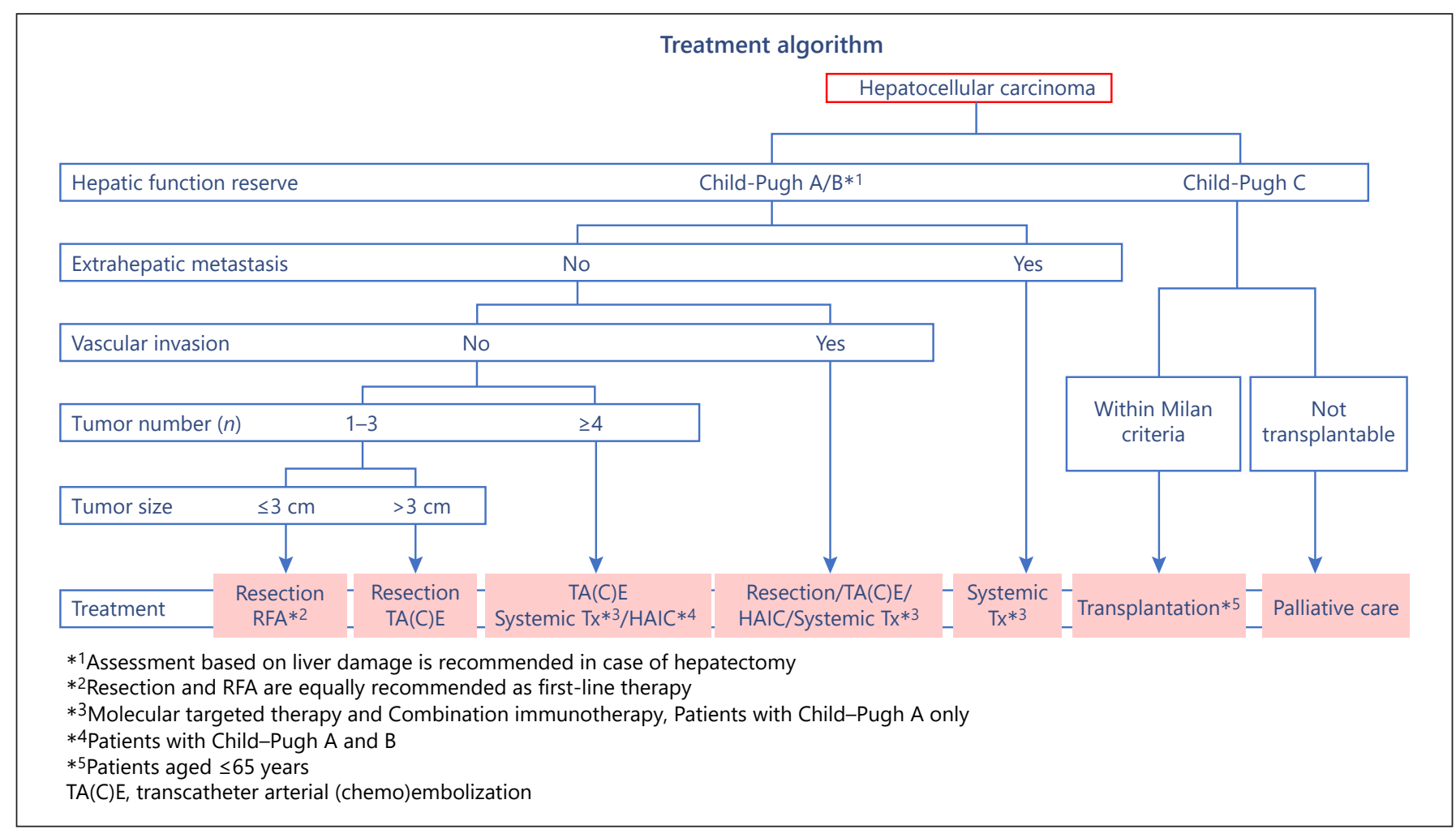

Fig. 11. Treatment algorithm. HCC, hepatocellular carcinoma; HAIC, hepatic arterial infusion chemotherapy; RFA, radiofrequency ablation; TACE, transarterial chemoembolization; RFA, radiofrequency ablation; Systemic Tx, molecular targeted therapy and/or combination immunotherapy. Reproduced with permission from the Japan Society of Hepatology [8], Kokudo et al. [9], and the Japan Society of Hepatology [17].

(5.9\%) of all 507 such transplants. Therefore, liver transplants from brain-dead donors accounted for only $1.7 \%$ of the 1,718 liver transplants performed for HCC. Moreover, because HCC patients must have decompensated cirrhosis to be eligible for liver transplantation from brain-dead donors, the target population is likely to include many patients with decompensated cirrhosis.

In the spring of 2019, the recipient selection criteria for liver transplantation from brain-dead donors were amended to prioritize patients based on the model for end-stage liver disease (MELD) score, rather than on medical urgency or waiting time. In this new system, an additional 2 points are added to the initial MELD score of HCC patients every 3 months; this is assigned at the time of transplant registration (every 6 months for diseases other than hepatoblastoma) to reduce dropouts during the waiting time. Therefore, there are likely to be more opportunities to perform liver transplantation from braindead donors in HCC patients. It should be noted that patients must undergo imaging studies every 3 months to ensure compliance with the Milan or 5-5-500 criteria.

\section{Treatment Algorithm for HCC Proposed by JSH}

\section{Consensus Statements}

85. The JSH treatment algorithm in the HCC practice manual is the same as the JSH Clinical Practice Guidelines for HCC 2017, Revised Edition.

The JSH treatment algorithm for HCC in this practice manual is basically the same as the JSH Clinical Practice Guidelines for HCC 2017, Revised Edition [9] (Fig. 11). Treatment strategies for HCC are based on 5 factors: (1) liver function, (2) extrahepatic spread, (3) vascular invasion, (4) number of tumors, and (5) tumor size. For the assessment of liver function, it is generally appropriate to use the globally accepted Child-Pugh classification. However, the JSH Clinical Practice Guidelines for HCC 2017, Revised Edition, recommend assessing the degree of liver damage, including the use of the ICG test only in patients considered for hepatectomy. Extrahepatic spread, vascular invasion, number of tumors, and tumor size are pretreatment prognostic factors and should be assessed using imaging studies such as CT and EOB-MRI. The use of 
EOB-MRI is usually recommended before the initial treatment for accurate staging because of its high detection rate. In patients classified as Child-Pugh class A or B with no extrahepatic spread or vascular invasion, the treatment strategy depends on the number of tumors. A slash within a treatment option indicates that the recommendation for one treatment is equal to that for the other treatment. In the absence of a slash, the treatment in the upper row has a higher recommendation than that in the lower row.

Treatment Strategies for Patients Classified as Child-

Pugh Class A or B with No Extrahepatic Spread or

Vascular Invasion

Patients with 1-3 Tumors

Hepatectomy or RFA is recommended for patients with 1-3 tumors and tumor diameter $\leq 3 \mathrm{~cm}$. The results of the SURF trial presented at the ASCO in June 2019 showed no difference in RFS after curative hepatectomy or RFA [102], suggesting that either hepatectomy or RFA may be selected for patients with $\leq 3$ tumors and tumor size $\leq 3 \mathrm{~cm}$. For any tumors $>3 \mathrm{~cm}$ in diameter, hepatectomy is recommended as first-line treatment for resectable disease and TACE as second-line treatment for unresectable disease (Fig. 11).

\section{Patients with $\geq 4$ Tumors}

In patients with $\geq 4$ tumors, TACE is recommended as first-line treatment and HAIC and systemic therapy are recommended as second-line treatment.

\section{Hepatic Arterial Infusion Chemotherapy}

The Clinical Practice Guidelines for HCC 2017, Revised Edition, [9] equally recommend HAIC and MTAs for patients with $\geq 4$ intrahepatic multiple HCCs; however, a sub-analysis in the SILIUS study [173] and results from a nationwide retrospective propensity matched study [175] suggest that systemic therapy and HAIC are equally effective in Child-Pugh class A patients with multiple tumors $(\geq 4)$. Therefore, because of its invasive nature, HAIC is not actively recommended as the first choice of treatment for Child-Pugh class A patients with $\geq 4$ tumors, and it is primarily recommended for ChildPugh class B patients.

The rationale behind recommending HAIC for HCCs with $\geq 4$ tumors in the Clinical Practice Guidelines for HCC 2017, Revised Edition, [9] is based on the results of the study by Nouso et al. [196], which primarily used Nation-wide follow-up survey data from the Liver Cancer Study Group of Japan. This study showed a survival ben-

JSH Consensus Statements and Recommendations on HCC efit for HAIC over no treatment. However, recent evidence from JAMA Oncology [174] and a sub-analysis of the SILIUS study [173] showed that HAIC is effective for HCCs with vascular invasion, whereas it is not actively recommended for multiple intrahepatic HCCs with $\geq 4$ tumors, as mentioned earlier. More specifically, because HAIC is only as effective as systemic therapy, the preferred treatment for patients with multiple intrahepatic HCCs classified as Child-Pugh class A is systemic therapy. Systemic therapy is recommended for TACE-unsuitable patients as well as TACE failure/refractory patients $[148,149]$.

\section{Systemic Therapy}

Systemic therapy is recommended for multiple intrahepatic HCCs or intermediate-stage HCC based on the SHARP study [177, 197], which reported an OS of 14.5 months with sorafenib and 10.2 months with placebo in patients with intermediate-stage $\mathrm{HCC}(\mathrm{HR}=0.52)$. These results were better than those obtained with sorafenib in advanced HCC (OS $=8.9$ months; $\mathrm{HR}=0.7)$. In the AsiaPacific study [178], the OS of patients with intermediatestage HCC was longer in those treated with sorafenib (14.3 months) than in those treated with placebo (8 months; HR $=0.45$ ), and it was also better than the survival outcome of patients with advanced HCC treated with sorafenib (median survival, 7.3 months). Thus, sorafenib is also recommended for intermediate-stage HCC.

Sub-analyses in the REFLECT study [139] showed an OS of 11.6 months with lenvatinib alone versus 23.0 months with lenvatinib followed by TACE and 10.1 months with sorafenib alone versus 19.6 months with sorafenib followed by TACE, suggesting an additional survival benefit of TACE after molecular targeted therapy. Similarly, sequential therapy with sorafenib followed by TACE compared with TACE alone showed better PFS in patients treated with sorafenib and TACE [130]. These results suggest that pretreatment with MTAs prior to TACE is a preferable option.

In a subgroup analysis of Japanese patients, lenvatinib was effective in the treatment of intermediate-stage HCC, showing a $62 \%$ response rate [140] and particularly long median survival. A recent report showed that pretreatment with lenvatinib followed by TACE resulted in better survival than TACE alone in patients who did not meet the up-to-7 criteria (median, 37.9 vs. 21.3 months, respectively) [149]. Based on these results, systemic therapy is recommended over TACE for multiple intrahepatic HCCs with high tumor burden $[148,149]$. TACE or 
HAIC is, of course, still recommended for multiple intrahepatic HCCs in Child-Pugh class B patients, as these patients are ineligible for systemic therapy.

\section{Treatment Strategy for HCC with Vascular Invasion}

In Europe and the USA, only systemic therapy is recommended for HCC with vascular invasion, whereas in Japan, TACE is recommended for HCC with minor portal vein invasion, such as $\mathrm{Vp}_{1}$ and $\mathrm{Vp}_{2}$, and is routinely performed. Hepatectomy is also recommended for HCC with portal or hepatic vein invasion based on propensity score-matched data from the Liver Cancer Study Group of Japan, which showed better outcomes in patients treated with hepatectomy than in unresectable cases $[198,199]$. HAIC is also recommended as an important option based on the results of propensity score matching of data from the Liver Cancer Study Group of Japan reported by Nouso et al.[196], a sub-analysis of the SILIUS study, and a recent report in JAMA Oncology by He et al. [174]. There is also strong evidence supporting the effectiveness of systemic therapy for HCC with vascular invasion, which is recommended as the standard of care worldwide. Thus, the 4 treatment modalities including TACE, hepatectomy, HAIC, and systemic therapy are equally recommended for HCC with vascular invasion (Fig. 11).

However, there are slight differences in the recommendations between these treatments; HAIC and systemic therapy are indicated for a large number of patients, whereas TACE and hepatectomy are indicated for more strictly selected populations defined by liver function and other factors. In these populations, patients who receive these treatments show better outcomes than those who do not.

For patients with vascular invasion, the first step is to determine eligibility for hepatectomy or TACE. If the patient is eligible, the next step is to perform either procedure; if not eligible, HAIC should be considered first and then systemic therapy. Specifically, whether hepatectomy is feasible needs to be considered first, and then whether TACE is possible to obtain a better outcome. In patients with major vascular invasion, such as $\mathrm{Vp}_{3}$ and $\mathrm{Vp}_{4}$, atezolizumab plus bevacizumab [187] or HAIC is the preferred option. For Child-Pugh class B patients, the first choice of treatment is HAIC. Systemic therapy is recommended only for Child-Pugh class A patients, whereas HAIC is applicable to Child-Pugh class B patients.
Treatment Strategy for Patients with Extrahepatic Spread

For patients with extrahepatic spread, systemic therapy is the only recommended treatment in those classified as Child-Pugh class A. For Child-Pugh class B patients in whom the intrahepatic lesion is considered a prognostic factor, selective TACE or HAIC may be actively considered (Fig. 11).

\section{Treatment Strategy for Child-Pugh Class C Patients}

For Child-Pugh class $\mathrm{C}$ patients, liver transplantation is recommended if applicable. Current indications for liver transplantation include not only patients who meet the Milan criteria, but also those who do not meet them with a tumor diameter $\leq 5 \mathrm{~cm}, \leq 5$ tumors, and AFP $<500 \mathrm{ng} /$ $\mathrm{mL}$ (the 5-5-500 criteria). The algorithm in the JSH Clinical Practice Guidelines for HCC 2017, Revised Edition, adopted these criteria, which are also applicable to livingdonor liver transplantation. Liver transplantation is basically indicated for patients aged $\leq 65$ years. Patients who do not meet the eligibility criteria for liver transplantation are provided palliative treatment. Many reports from Japan have suggested the survival benefit of locoregional therapies and TACE in strictly selected HCC patients with Child-Pugh class C liver function [116-121], although this has not yet been recommended in guidelines (Fig. 11).

\section{Conclusion}

The fourth edition of the Clinical Practice Manual for HCC [17], which is the basis for this article, was written by experts in their respective fields (online suppl. Table 1) in accordance with the JSH Clinical Practice Guidelines for HCC 2017 [8]. Because the JSH Clinical Practice Guidelines are based on original articles with high levels of evidence, expert opinions on HCC management in clinical practice or consensus on newly developed treatments are not included. In this article, we included newly developed clinical practices that are relatively common among Japanese experts in this field, although there are few papers with a high level of evidence, and these practices are likely to be incorporated into guidelines in the future.

To write this article, coauthors from different institutions drafted the content and then critically reviewed each other's work. The revised content was then critically reviewed by the Board of Directors and the Planning and Public Relations Committee of JSH before publication to 
confirm the consensus statements and recommendations issued from JSH.

The consensus statements and recommendations presented in this report represent measures actually being instituted at the highest level HCC treatment centers in Japan. The outcomes of HCC treatment in Japan are reported to be the best in the world [200-203], and we hope that this article provides insight into the actual situation of HCC practice in Japan, thereby affecting the global practice pattern in the management of HCC.

\section{Conflict of Interest Statement}

M.K. received honoraria from Eisai, Bayer, MSD, Bristol-Myers Squibb, Lilly, and EA Pharma and grants from Gilead Sciences, Taiho, Sumitomo Dainippon Pharma, Takeda, Otsuka, EA Pharma, Abbvie, and Eisai and has had an advisory role in Eisai, Ono, MSD, Bristol-Myers Squibb, and Roche. Y.K. received honoraria from Esiai. K.H. received honoraria from Taiho, Chugai, and Takeda. R.T. received honoraria from Abbvie, Bayer, Chugai, Eisai, Fujifilm Wako, GE Healthcare, Gilead Sciences, Medtronic, MSD, Otsuka, Shionogi, and Sumitomo Dainippon. K.K. has no conflicts of interest to declare. S.S. has no conflicts of interest to declare. H.T. received honoraria from AbbVie, MSD, and Bayer. Y.I. has no conflicts of interest to declare. A.H. received honoraria from Eisai, Bayer, and Otsuka. M.I. received honoraria from Eisai, Bayer, and Lilly and research funding from Bayer, Eisai, Ono, Bristol Myers Squibb, AstraZeneca, Chugai, Merck Serono, Novartis Pharma, and MSD. N.I. received honoraria from Bayer and Eisai. M.M. received honoraria from Eisai, Bayer, and Lilly. S.O. received honoraria from Bayer, Eisai, and Eli Lilly; consulting or advisory fees from Bayer, Eisai, Merck \& Co., Inc., Chugai Pharma, Eli Lilly, and AstraZeneca; and research grants from Bayer, Eisai, and Eli Lilly. Y.M. has no conflicts of interest to declare. K.U. received honoraria from Eisai and Eli Lilly. T.M. has no conflicts of interest to declare. S.M. received honoraria from Eisai, Bayer, Guerbet, Asahi Intecc, Philips, Canon, Piolax, Daiichi-Sankyo, Fujiyakuhin, and Eli Lilly. O.N. has no conflicts of interest to declare. H.Y. has no conflicts of interest to declare. M.S. received grants from Eisai, Olympus, Fujifilm, and CYTLIMIC. E.H. has no conflicts of interest to declare. M.S. received grants from EA Pharma, Eisai, Covidien Japan, Novartis Pharma, Taiho, Chugai, Bayer, Astellas, Ono, and Takeda. N.K. has no conflicts of interest to declare. S.M. has no conflicts of interest to declare. T.T. received honoraria from Gilead Sciences, AbbVie, and MAD and grant/research support from Gilead Sciences, AbbVie, MSD, Janssen, Eisai, EA Pharma, and Otsuka.

\section{Funding Sources}

The authors did not receive any funding.

\section{Author Contributions}

M.K. conceived, wrote, and approved the final manuscript. Y.K., K.H., R.T., K.K., S.S., H.T., Y.I., A.H., M.I., N.I., M.M., S.O., Y.M., K.U., T.M., S.M., O.N., H.Y., M.S., E.H., M.S., N.K., S.M., and T.T. conceived, contributed, reviewed, provided critical comments, and approved the final manuscript.

\section{References}

1 The Japan Society of Hepatology: evidencebased clinical practice guideline for hepatocellular carcinoma. Tokyo: Kanehara; 2005 (in Japanese).

2 Makuuchi M, Kokudo N. Clinical practice guidelines for hepatocellular carcinoma: the first evidence based guidelines from Japan. World J Gastroenterol. 2006;12(5):828-9.

3 Makuuchi M, Kokudo N, Arii S, Futagawa S, Kaneko S, Kawasaki S, et al. Development of evidence-based clinical guidelines for the diagnosis and treatment of hepatocellular carcinoma in Japan. Hepatol Res. 2008;38(1):3751.

4 The Japan Society of Hepatology: evidencebased clinical practice guideline for hepatocellular carcinoma (2009 updated version). Tokyo: Kanehara; 2009. (in Japanese).

5 Makuuchi M, Kokudo N. Clinical practice guidelines for hepatocellular carcinoma: the Japan Society of Hepatology 2009 update. Hepatol Res. 2010;40(Suppl 1):2-144.
6 The Japan Society of Hepatology: evidencebased clinical practice guideline for hepatocellular carcinoma (2013 updated version). Tokyo: Kanehara; 2013. (in Japanese).

7 Kokudo N, Hasegawa K, Akahane M, Igaki H, Izumi N, Ichida T, et al. Evidence-based Clinical Practice Guidelines for Hepatocellular Carcinoma: the Japan Society of Hepatology 2013 update (3rd JSH-HCC Guidelines). Hepatol Res. 2015;45(2):123-7.

8 The Japan Society of Hepatology: evidencebased clinical practice guideline for hepatocellular carcinoma (2017 updated version). Tokyo: Kanehara; 2017 (in Japanese).

9 Kokudo N, Takemura N, Hasegawa K, Takayama T, Kubo S, Shimada M, et al. Clinical practice guidelines for hepatocellular carcinoma: the Japan Society of Hepatology 2017 (4th JSH-HCC guidelines) 2019 update. Hepatol Res. 2019;49:1109-13.

10 The Japan Society of Hepatology: clinical practice manual for hepatocellular carcinoma. Tokyo: Igakushoin; 2007 (in Japanese).
11 Kudo M, Okanoue T; Japan Society of Hepatology. Management of hepatocellular carcinoma in Japan: consensus-based clinical practice manual proposed by the Japan Society of Hepatology. Oncology. 2007;72(Suppl $1): 2-15$.

12 The Japan Society of Hepatology: clinical practice manual for hepatocellular carcinoma. 2nd ed. Tokyo: Igakushoin; 2010 (in Japanese).

13 Kudo M, Izumi N, Kokudo N, Matsui O, Sakamoto M, Nakashima O, et al. Management of hepatocellular carcinoma in Japan: Consensus-Based Clinical Practice Guidelines proposed by the Japan Society of Hepatology (JSH) 2010 updated version. Dig Dis. 2011;29(3):339-64.

14 The Japan Society of Hepatology: clinical practice manual for hepatocellular carcinoma. 3rd ed. Tokyo: Igakushoin; 2015 (in Japanese).
JSH Consensus Statements and Recommendations on HCC
Liver Cancer 2021;10:181-223

DOI: $10.1159 / 000514174$ 
15 Kudo M, Matsui O, Izumi N, Iijima H, Kadoya $\mathrm{M}$, Imai $\mathrm{Y}$, et al. JSH consensus-based clinical practice guidelines for the management of hepatocellular carcinoma: 2014 update by the liver cancer study group of Japan. Liver Cancer. 2014;3(3-4):458-68.

16 Kudo M, Matsui O, Izumi N, Kadoya M, Okusaka T, Miyayama S, et al. Transarterial chemoembolization failure/refractoriness: JSHLCSGJ criteria 2014 update. Oncology. 2014; 87(Suppl 1):22-31.

17 The Japan Society of Hepatology: clinical practice manual for hepatocellular carcinoma. 4th ed. Tokyo: Igakushoin; 2020 (in Japanese).

18 Sakamoto M, Hirohashi S, Shimosato Y. Early stages of multistep hepatocarcinogenesis: adenomatous hyperplasia and early hepatocellular carcinoma. Hum Pathol. 1991;22(2) 172-8.

19 Nakashima O, Sugihara S, Kage M, Kojiro M. Pathomorphologic characteristics of small hepatocellular carcinoma: a special reference to small hepatocellular carcinoma with indistinct margins. Hepatology. 1995;22(1):101-5.

20 The Liver Cancer Study Group of Japan. The general rules for the clinical and pathological study of primary cancer (6th ed., revised version). Tokyo: Kanehara; 2019 (in Japanese).

21 Kondo F, Kondo Y, Nagato Y, Tomizawa M, Wada K. Interstitial tumour cell invasion in small hepatocellular carcinoma. Evaluation in microscopic and low magnification views. J Gastroenterol Hepatol. 1994;9(6):604-12.

22 International Consensus Group for Hepatocellular Neoplasia. Pathologic diagnosis of early hepatocellular carcinoma: a report of the International consensus group for hepatocellular neoplasia. Hepatology. 2009;49(2):65864.

23 Kutami R, Nakashima Y, Nakashima O, Shiota K, Kojiro M. Pathomorphologic study on the mechanism of fatty change in small hepatocellular carcinoma of humans. J Hepatol. 2000;33(2):282-9.

24 Kitao A, Zen Y, Matsui O, Gabata T, Kobayashi S, Koda W, et al. Hepatocellular carcinoma: signal intensity at gadoxetic acid-enhanced MR imaging: correlation with molecular transporters and histopathologic features. Radiology. 2010;256(3):817-26.

25 Nakashima Y, Nakashima O, Tanaka M, Okuda K, Nakashima M, Kojiro M. Portal vein invasion and intrahepatic micrometastasis in small hepatocellular carcinoma by gross type. Hepatol Res. 2003;26(2):142-7.

26 Kudo M. Multistep human hepatocarcinogenesis: correlation of imaging with pathology. J Gastroenterol. 2009;44(Suppl 19):112-8.

27 Yamaguchi R, Nakashima O, Yano H, Kutami R, Kusaba A, Kojiro M. Hepatocellular carcinoma with sarcomatous change. Oncol Rep. 1997;4(3):525-9

28 Kudo M. Atypical large well-differentiated hepatocellular carcinoma with benign nature: a new clinical entity. Intervirology. 2004; $47(3-5): 227-37$.
29 Nault JC, Paradis V, Cherqui D, Vilgrain V, Zucman-Rossi J. Molecular classification of hepatocellular adenoma in clinical practice. J Hepatol. 2017;67(5):1074-83.

30 Taketa K. Alpha-fetoprotein: reevaluation in hepatology. Hepatology. 1990;12(6):1420-32.

31 Aoyagi Y, Saitoh A, Suzuki Y, Igarashi K, Oguro M, Yokota T, et al. Fucosylation index of alpha-fetoprotein, a possible aid in the early recognition of hepatocellular carcinoma in patients with cirrhosis. Hepatology. 1993; 17(1):50-2.

32 Liebman HA, Furie BC, Tong MJ, Blanchard RA, Lo KJ, Lee SD, et al. Des-gamma-carboxy (abnormal) prothrombin as a serum marker of primary hepatocellular carcinoma. N Engl J Med. 1984;310(22):1427-31.

33 Fujiyama S, Morishita T, Hashiguchi O, Sato T. Plasma abnormal prothrombin (des-gamma-carboxy prothrombin) as a marker of hepatocellular carcinoma. Cancer. 1988;61(8): 1621-8.

34 Tanaka S, Kitamra T, Fujita M, Kasugai H, Inoue A, Ishiguro S. Small hepatocellular carcinoma: differentiation from adenomatous hyperplastic nodule with color Doppler flow imaging. Radiology. 1992;182(1):161-5.

35 Sasaki S, Iijima H, Moriyasu F, Waki H. Definition of contrast enhancement phases of the liver using a perfluoro-based microbubble agent, perflubutane microbubbles. Ultrasound Med Biol. 2009;35:1819-27.

36 Moriyasu F, Itoh K. Efficacy of perflubutane microbubble-enhanced ultrasound in the characterization and detection of focal liver lesions: phase 3 multicenter clinical trial. AJR Am J Roentgenol. 2009;193(1):86-95.

37 Claudon M, Dietrich CF, Choi BI, Cosgrove DO, Kudo M, Nolsøe CP, et al. Guidelines and good clinical practice recommendations for Contrast Enhanced Ultrasound (CEUS) in the liver: update 2012: a WFUMB-EFSUMB initiative in cooperation with representatives of AFSUMB, AIUM, ASUM, FLAUS and ICUS. Ultrasound Med Biol. 2013;39(2):187210.

38 Kudo M, Hatanaka K, Kumada T, Toyoda H, Tada T. Double-contrast ultrasound: a novel surveillance tool for hepatocellular carcinoma. Am J Gastroenterol. 2011;106(2):368-70.

39 Kudo M, Ueshima K, Osaki Y, Hirooka M, Imai $\mathrm{Y}$, Aso K, et al. B-mode ultrasonography versus contrast-enhanced ultrasonography for surveillance of hepatocellular carcinoma: a prospective multicenter randomized controlled trial. Liver Cancer. 2019;8(4):271-80.

40 Kudo M, Hatanaka K, Maekawa K. Newly developed novel ultrasound technique, defect reperfusion ultrasound imaging, using sonazoid in the management of hepatocellular carcinoma. Oncology. 2010;78(Suppl 1):405.

41 Kudo M. Defect Reperfusion Imaging with Sonazoid: a breakthrough in hepatocellular carcinoma. Liver Cancer. 2016;5:1-7.
42 Dietrich CF, Nolsoe CP, Barr RG, Berzigotti A, Bums PN, Cantisani V, et al. Guidelines and good clinical practice recommendations for contrast enhanced ultrasound (CEUS) in the liver-update 2020: WFUMB in cooperation with EFSUMB, AFSUMB, AIUM, and FLAUS. Ultrasound Med Biol. 2020 Oct;46: 2579-604.

43 Dietrich CF, Nolsoe CP, Barr RG, Berzigotti A, Bums PN, Cantisani V, et al. Guidelines and good clinical practice recommendations for contrast enhanced ultrasound (CEUS) in the liver-update 2020: WFUMB in cooperation with EFSUMB, AFSUMB, AIUM, and FLAUS. Ultrasound Med Biol. 2020 Oct;46: 2579-604. Epub ahead of print.

44 Hatanaka K, Kudo M, Minami Y, Ueda T, Tatsumi C, Kitai S, et al. Differential diagnosis of hepatic tumors: value of contrast-enhanced harmonic sonography using the newly developed contrast agent, Sonazoid. Intervirology. 2008;51:S61-69.

45 Hatanaka K, Minami Y, Kudo M, Inoue T, Chung H, Haji S. The gross classification of hepatocellular carcinoma: usefulness of contrast-enhanced US. J Clin Ultrasound. 2014; 42(1):1-8.

46 Iwamoto T, Imai Y, Kogita S, Igura T, Sawai $\mathrm{Y}$, Fukuda $\mathrm{K}$, et al. Comparison of contrastenhanced ultrasound and gadolinium-ethoxybenzyl-diethylenetriamine pentaacetic acid-enhanced MRI for the diagnosis of macroscopic type of hepatocellular carcinoma. Dig Dis. 2016;34(6):679-86.

47 Korenaga K, Korenaga M, Furukawa M, Yamasaki T, Sakaida I. Usefulness of Sonazoid contrast-enhanced ultrasonography for hepatocellular carcinoma: comparison with pathological diagnosis and superparamagnetic iron oxide magnetic resonance images. J Gastroenterol. 2009;44(7):733-41.

48 Tanaka H, Iijima H, Higashiura A, Yoh K, Ishii A, Takashima T, et al. New malignant grading system for hepatocellular carcinoma using the Sonazoid contrast agent for ultrasonography. J Gastroenterol. 2014;49(4):755-63.

49 Minami T, Minami Y, Chishina H, Arizumi T, Takita M, Kitai S, et al. Combination guidance of contrast-enhanced US and fusion imaging in radiofrequency ablation for hepatocellular carcinoma with poor conspicuity on contrast-enhanced US/fusion imaging. Oncology. 2014;87(Suppl 1):55-62.

50 Manini MA, Sangiovanni A, Fornari F, Piscaglia F, Biolato M, Fanigliulo L, et al. Clinical and economical impact of 2010 AASLD guidelines for the diagnosis of hepatocellular carcinoma. J Hepatol. 2014;60(5):995-1001.

51 Murakami T, Kim T, Takamura M, Hori M, Takahashi S, Federle MP, et al. Hypervascular hepatocellular carcinoma: detection with double arterial phase multi-detector row helical CT. Radiology. 2001;218(3):763-7.

52 Kadoya M, Matsui O, Takashima T, Nonomura A. Hepatocellular carcinoma: correlation of MR imaging and histopathologic findings. Radiology. 1992;183(3):819-25. 
53 Motosugi U, Ichikawa T, Sou H, Sano K, Tominaga L, Muhi A, et al. Distinguishing hypervascular pseudolesions of the liver from hypervascular hepatocellular carcinomas with gadoxetic acid-enhanced MR imaging. Radiology. 2010;256(1):151-8.

54 Sofue K, Marin D, Jaffe TA, Nelson RC, Bashir MR. Can combining triple-arterial phase acquisition with fluoroscopic triggering provide both optimal early and late hepatic arterial phase images during gadoxetic acid-enhanced MRI? J Magn Reson Imaging. 2016;43(5):1073-81.

55 Lee YJ, Lee JM, Lee JS, Lee HY, Park BH, Kim $\mathrm{YH}$, et al. Hepatocellular carcinoma: diagnostic performance of multidetector $\mathrm{CT}$ and $\mathrm{MR}$ imaging-a systematic review and meta-analysis. Radiology. 2015;275(1):97-109.

56 Imai Y, Murakami T, Yoshida S, Nishikawa M, Ohsawa M, Tokunaga K, et al. Superparamagnetic iron oxide-enhanced magnetic resonance images of hepatocellular carcinoma: correlation with histological grading. Hepatology. 2000;32(2):205-12.

57 Duncan JK, Ma N, Vreugdenburg TD, Cameron AL, Maddern G. Gadoxetic acid-enhanced MRI for the characterization of hepatocellular carcinoma: a systematic review and meta-analysis. J Magn Reson Imaging. 2017; 45(1):281-90.

58 Kitao A, Matsui O, Yoneda N, Kozaka K, Kobayashi S, Sanada J, et al. Hepatocellular carcinoma with beta-catenin mutation: imaging and pathologic characteristics. Radiology. 2015;275:708-17

59 Kudo M. Gd-EOB-DTPA-MRI could predict $\mathrm{WNT} / \beta$-catenin mutation and resistance to immune checkpoint inhibitor therapy in hepatocellular carcinoma. Liver Cancer. 2020 Sep;9(5):479-90.

60 Sano K, Ichikawa T, Motosugi U, Sou H, Muhi AM, Matsuda M, et al. Imaging study of early hepatocellular carcinoma: usefulness of gadoxetic acid-enhanced MR imaging. Radiology. 2011;261(3):834-44.

61 Goshima S, Kanematsu M, Watanabe H, Kondo H, Shiratori Y, Onozuka M, et al. Hepatic hemangioma and metastasis: differentiation with gadoxetate disodium-enhanced 3-T MRI. AJR Am J Roentgenol. 2010;195(4): 941-6.

62 Matsui O, Kadoya M, Kameyama T, Yoshikawa J, Takashima T, Nakanuma Y, et al. Benign and malignant nodules in cirrhotic livers: distinction based on blood supply. Radiology. 1991;178(2):493-7.

63 Hayashi M, Matsui O, Ueda K, Kawamori Y, Kadoya M, Yoshikawa J, et al. Correlation between the blood supply and grade of malignancy of hepatocellular nodules associated with liver cirrhosis: evaluation by CT during intraarterial injection of contrast medium. AJR Am J Roentgenol. 1999;172(4):969-76.
64 Narita M, Hatano E, Arizono S, MiyagawaHayashino A, Isoda H, Kitamura K, et al. Expression of OATP1B3 determines uptake of Gd-EOB-DTPA in hepatocellular carcinoma. J Gastroenterol. 2009;44(7):793-8.

65 Kitao A, Matsui O, Yoneda N, Kozaka K, Shinmura R, Koda W, et al. The uptake transporter OATP8 expression decreases during multistep hepatocarcinogenesis: correlation with gadoxetic acid enhanced MR imaging. Eur Radiol. 2011;21(10):2056-66.

66 Kogita S, Imai Y, Okada M, Kim T, Onishi H, Takamura $\mathrm{M}$, et al. Gd-EOB-DTPA-enhanced magnetic resonance images of hepatocellular carcinoma: correlation with histological grading and portal blood flow. Eur Radiol. 2010;20(10):2405-13.

67 Kumada T, Toyoda H, Tada T, Sone Y, Fujimori M, Ogawa S, et al. Evolution of hypointense hepatocellular nodules observed only in the hepatobiliary phase of gadoxetate disodium-enhanced MRI. AJR Am J Roentgenol. 2011;197(1):58-63.

68 Motosugi U, Ichikawa T, Sano K, Sou H, Onohara K, Muhi A, et al. Outcome of hypovascular hepatic nodules revealing no gadoxetic acid uptake in patients with chronic liver disease. J Magn Reson Imaging. 2011;34(1):8894.

69 Hyodo T, Murakami T, Imai Y, Okada M, Hori M, Kagawa Y, et al. Hypovascular nodules in patients with chronic liver disease: risk factors for development of hypervascular hepatocellular carcinoma. Radiology. 2013; 266(2):480-90.

70 EASL Clinical Practice Guidelines. Management of hepatocellular carcinoma. J Hepatol. 2018;69:182-236.

71 Marrero JA, Kulik LM, Sirlin CB, Zhu AX, Finn RS, Abecassis MM, et al. Diagnosis, staging, and management of hepatocellular carcinoma: 2018 practice guidance by the American Association for the study of liver diseases. Hepatology. 2018;68(2):723-50.

72 Takayama T, Makuuchi M, Hirohashi S, Sakamoto M, Yamamoto J, Shimada K, et al. Early hepatocellular carcinoma as an entity with a high rate of surgical cure. Hepatology. 1998;28(5):1241-6.

73 Midorikawa Y, Takayama T, Shimada K, Nakayama $\mathrm{H}$, Higaki T, Moriguchi M, et al. Marginal survival benefit in the treatment of early hepatocellular carcinoma. J Hepatol. 2013; 58(2):306-11

74 Kudo M, Matsui O, Izumi N, Iijima H, Kadoya M, Imai Y. Surveillance and diagnostic algorithm for hepatocellular carcinoma proposed by the Liver Cancer Study Group of Japan: 2014 update. Oncology. 2014;87(Suppl 1):721.

75 Ohama H, Imai Y, Nakashima O, Kogita S, Takamura M, Hori M, et al. Images of Sonazoid-enhanced ultrasonography in multistep hepatocarcinogenesis: comparison with Gd-EOB-DTPA-enhanced MRI. J Gastroenterol. 2014;49(6):1081-93.
76 Inoue T, Hyodo T, Korenaga K, Murakami T, Imai $Y$, Higaki A, et al. Kupffer phase image of Sonazoid-enhanced US is useful in predicting a hypervascularization of non-hypervascular hypointense hepatic lesions detected on Gd-EOB-DTPA-enhanced MRI: a multicenter retrospective study. J Gastroenterol. 2016;51(2):144-52.

77 Omata M, Cheng AL, Kokudo N, Kudo M, Lee JM, Jia J, et al. Asia-Pacific clinical practice guidelines on the management of hepatocellular carcinoma: a 2017 update. Hepatol Int. 2017;11(4):317-70.

78 Okuda K, Ohtsuki T, Obata H, Tomimatsu M, Okazaki N, Hasegawa H, et al. Natural history of hepatocellular carcinoma and prognosis in relation to treatment. Study of 850 patients. Cancer. 1985;56(4):918-28.

79 Llovet JM, Brú C, Bruix J. Prognosis of hepatocellular carcinoma: the BCLC staging classification. Semin Liver Dis. 1999;19(3):329_ 38.

80 Forner A, Reig M, Bruix J. Hepatocellular carcinoma. Lancet. 2018;391(10127):1301-14.

81 A new prognostic system for hepatocellular carcinoma: a retrospective study of 435 patients: the Cancer of the Liver Italian Program (CLIP) investigators. Hepatology. 1998;28(3): 751-5.

82 Kudo M, Chung H, Osaki Y. Prognostic staging system for hepatocellular carcinoma (CLIP score): its value and limitations, and a proposal for a new staging system, the Japan Integrated Staging Score (JIS score). J Gastroenterol. 2003;38(3):207-15.

83 Kudo M, Chung H, Haji S, Osaki Y, Oka H, Seki T, et al. Validation of a new prognostic staging system for hepatocellular carcinoma: the JIS score compared with the CLIP score. Hepatology. 2004;40(6):1396-405.

84 Nanashima A, Sumida Y, Abo T, Shindou H, Fukuoka H, Takeshita H, et al. Modified Japan Integrated Staging is currently the best available staging system for hepatocellular carcinoma patients who have undergone hepatectomy. J Gastroenterol. 2006;41(3):250-6.

85 Ikai I, Takayasu K, Omata M, Okita K, Nakanuma Y, Matsuyama Y, et al. A modified Japan Integrated Stage score for prognostic assessment in patients with hepatocellular carcinoma. J Gastroenterol. 2006;41(9):88492.

86 Kitai S, Kudo M, Minami Y, Ueshima K, Chung H, Hagiwara S, et al. A new prognostic staging system for hepatocellular carcinoma: value of the biomarker combined Japan integrated staging score. Intervirology. 2008;51: S86-94.

87 Tateishi R, Yoshida H, Shiina S, Imamura H, Hasegawa K, Teratani T, et al. Proposal of a new prognostic model for hepatocellular carcinoma: an analysis of 403 patients. Gut. 2005; 54(3):419-25.
JSH Consensus Statements and Recommendations on HCC

Liver Cancer 2021;10:181-223 
88 Toyoda H, Kumada T, Osaki Y, Oka H, Urano F, Kudo M, et al. Staging hepatocellular carcinoma by a novel scoring system (BALAD score) based on serum markers. Clin Gastroenterol Hepatol. 2006;4(12):1528-36.

89 Toyoda H, Tada T, Johnson PJ, Izumi N, Kadoya M, Kaneko S, et al. Validation of serological models for staging and prognostication of HCC in patients from a Japanese nationwide survey. J Gastroenterol. 2017;52: 1112-21.

90 Caviglia GP, Abate ML, Petrini E, Gaia S, Rizzetto M, Smedile A. Highly sensitive alpha-fetoprotein, Lens culinaris agglutinin-reactive fraction of alpha-fetoprotein and desgamma-carboxyprothrombin for hepatocellular carcinoma detection. Hepatol Res. 2016; 46(3):E130-5.

91 Hiraoka A, Kumada T, Michitaka K, Toyoda $\mathrm{H}$, Tada T, Ueki H, et al. Usefulness of albumin-bilirubin grade for evaluation of prognosis of 2584 Japanese patients with hepatocellular carcinoma. J Gastroenterol Hepatol. 2016;31(5):1031-6.

92 Hiraoka A, Kumada T, Tsuji K, Takaguchi K, Itobayashi E, Kariyama K, et al. Validation of modified ALBI grade for more detailed assessment of hepatic function in hepatocellular carcinoma patients: a multicenter analysis. Liver Cancer. 2019;8(2):121-9.

93 Yau T, Tang VY, Yao TJ, Fan ST, Lo CM, Poon RT. Development of Hong Kong Liver Cancer staging system with treatment stratification for patients with hepatocellular carcinoma. Gastroenterology. 2014;146(7):1691700.e3.

94 Liu PH, Hsu CY, Lee YH, Su CW, Hsia CY, Huang $\mathrm{YH}$, et al. Hong Kong liver cancer staging system is associated with better performance for hepatocellular carcinoma: special emphasis on viral etiology. Medicine. 2015;94:e1772.

95 Hiraoka A, Kumada T, Kudo M, Hirooka M, Tsuji K, Itobayashi E, et al. Albumin-Bilirubin (ALBI) Grade as part of the evidence-based clinical practice guideline for $\mathrm{HCC}$ of the Japan Society of Hepatology: a comparison with the liver damage and Child-Pugh classifications. Liver Cancer. 2017;6(3):204-15.

96 Kudo M, Izumi N, Kubo S, Kokudo N, Sakamoto M, Shiina S, et al. Report of the 20th Nationwide follow-up survey of primary liver cancer in Japan. Hepatol Res. 2020;50(1):1546.

97 Kakeji Y, Takahashi A, Udagawa H, Unno M, Endo I, Kunisaki C, et al. Surgical outcomes in gastroenterological surgery in Japan: report of National Clinical database 2011-2016 Ann Gastroenterol Surg. 2018;2(1):37-54.

98 Ishizawa $\mathrm{T}$, Hasegawa K, Aoki T, Takahashi M, Inoue Y, Sano K, et al. Neither multiple tumors nor portal hypertension are surgical contraindications for hepatocellular carcinoma. Gastroenterology. 2008;134(7):1908-16.
99 Shiina S, Teratani T, Obi S, Sato S, Tateishi $\mathrm{R}$, Fujishima T, et al. A randomized controlled trial of radiofrequency ablation with ethanol injection for small hepatocellular carcinoma. Gastroenterology. 2005;129(1): 122-30.

100 Chen MS, Li JQ, Zheng Y, Guo RP, Liang $\mathrm{HH}$, Zhang YQ, et al. A prospective randomized trial comparing percutaneous local ablative therapy and partial hepatectomy for small hepatocellular carcinoma. Ann Surg. 2006;243(3):321-8.

101 Huang J, Yan L, Cheng Z, Wu H, Du L, Wang J, et al. A randomized trial comparing radiofrequency ablation and surgical resection for HCC conforming to the Milan criteria. Ann Surg. 2010;252(6):903-12.

102 Izumi N, Hasegawa K, Nishioka Y, Takayama T, Yamanaka N, Masatoshi Kudo M, et al. A multicenter randomized controlled trial to evaluate the efficacy of surgery vs. radiofrequency ablation for small hepatocellular carcinoma (SURF trial). J Clin Oncol. 2019;37(Suppl 15):4002. [ASCO 2019, LBA]

103 Nouso K, Kariyama K, Nakamura S, Oonishi A, Wakuta A, Oyama A, et al. Application of radiofrequency ablation for the treatment of intermediate-stage hepatocellular carcinoma. J Gastroenterol Hepatol. 2017;32(3): 695-700.

104 Bolondi L, Burroughs A, Dufour JF, Galle PR, Mazzaferro V, Piscaglia F, et al. Heterogeneity of patients with intermediate (BCLC B) hepatocellular carcinoma: proposal for a subclassification to facilitate treatment decisions. Semin Liver Dis. 2012;32(4):348-59.

105 Yin XY, Xie XY, Lu MD, Xu HX, Xu ZF, Kuang M, et al. Percutaneous thermal ablation of medium and large hepatocellular carcinoma: long-term outcome and prognostic factors. Cancer. 2009;115(9):1914-23.

106 Shen A, Zhang H, Tang C, Chen Y, Wang Y, Zhang $C$, et al. Systematic review of radiofrequency ablation versus percutaneous ethanol injection for small hepatocellular carcinoma up to $3 \mathrm{~cm}$. J Gastroenterol Hepatol. 2013;28(5):793-800.

107 Koda M, Murawaki Y, Hirooka Y, Kitamoto M, Ono M, Sakaeda H, et al. Complications of radiofrequency ablation for hepatocellular carcinoma in a multicenter study: an analysis of 16346 treated nodules in 13283 patients. Hepatol Res. 2012;42(11):1058-64.

108 Maeda M, Saeki I, Sakaida I, Aikata H, Araki Y, Ogawa C, et al. Complications after radiofrequency ablation for hepatocellular carcinoma: a multicenter study involving 9,411 Japanese patients. Liver Cancer. 2020;9(1): 50-62.

109 Kasugai H, Osaki Y, Oka H, Kudo M, Seki T. Severe complications of radiofrequency ablation therapy for hepatocellular carcinoma: an analysis of 3,891 ablations in 2,614 patients. Oncology. 2007;72(Suppl 1):72-5.
110 Yamada R, Sato M, Kawabata M, Nakatsuka H, Nakamura K, Takashima S. Hepatic artery embolization in 120 patients with unresectable hepatoma. Radiology. 1983;148(2): 397-401.

111 Ohishi H, Uchida H, Yoshimura H, Ohue S, Ueda J, Katsuragi M, et al. Hepatocellular carcinoma detected by iodized oil. Use of anticancer agents. Radiology. 1985;154(1):259.

112 Matsui O, Miyayama S, Sanada J, Kobayashi S, Khoda W, Minami T, et al. Interventional oncology: new options for interstitial treatments and intravascular approaches: superselective TACE using iodized oil for HCC: rationale, technique and outcome. J Hepatobiliary Pancreat Sci. 2010;17(4):407-9.

113 Miyayama S, Matsui O. Superselective conventional transarterial chemoembolization for hepatocellular carcinoma: rationale, technique, and outcome. J Vasc Interv Radiol. 2016;27(9):1269-78.

114 Miyayama S, Matsui O, Yamashiro M, Ryu Y, Kaito K, Ozaki K, et al. Ultraselective transcatheter arterial chemoembolization with a 2 -f tip microcatheter for small hepatocellular carcinomas: relationship between local tumor recurrence and visualization of the portal vein with iodized oil. J Vasc Interv Radiol. 2007;18(3):365-76.

115 Lammer J, Malagari K, Vogl T, Pilleul F, Denys A, Watkinson A, et al. Prospective randomized study of doxorubicin-eluting-bead embolization in the treatment of hepatocellular carcinoma: results of the PRECISION V study. Cardiovasc Intervent Radiol. 2010; 33(1):41-52.

116 Nouso K, Kokudo N, Tanaka M, Kuromatsu R, Nishikawa H, Toyoda H, et al. Treatment of hepatocellular carcinoma with ChildPugh C cirrhosis. Oncology. 2014;87(Suppl 1):99-103.

117 Kitai S, Kudo M, Nishida N, Izumi N, Sakamoto M, Matsuyama Y, et al. Survival benefit of locoregional treatment for hepatocellular carcinoma with advanced liver cirrhosis. Liver Cancer. 2016;5(3):175-89.

118 Kudo M, Osaki Y, Matsunaga T, Kasugai H, Oka H, Seki T. Hepatocellular carcinoma in Child-Pugh C cirrhosis: prognostic factors and survival benefit of nontransplant treatments. Dig Dis. 2013;31(5-6):490-8.

119 Nouso K, Ito Y, Kuwaki K, Kobayashi Y, Nakamura S, Ohashi Y, et al. Prognostic factors and treatment effects for hepatocellular carcinoma in Child C cirrhosis. Br J Cancer. 2008;98(7):1161-5.

120 Nishikawa H, Kita R, Kimura T, Ohara Y, Takeda H, Sakamoto A, et al. Clinical efficacy of non-transplant therapies in patients with hepatocellular carcinoma with ChildPugh C liver cirrhosis. Anticancer Res. 2014; 34(6):3039-44. 
121 Toyoda H, Kumada T, Kiriyama S, Sone Y, Tanikawa M, Hisanaga $Y$, et al. Impact of tumor factors on the prognosis of patients with advanced cirrhosis (Child-Pugh class $\mathrm{C}$ ) and hepatocellular carcinoma. J Gastroenterol Hepatol. 2005;20(6):963-5.

122 Golfieri R, Giampalma E, Renzulli M, Cioni R, Bargellini I, Bartolozzi C, et al. Randomised controlled trial of doxorubicineluting beads vs conventional chemoembolisation for hepatocellular carcinoma. Br J Cancer. 2014;111(2):255-64.

123 Facciorusso A, Di Maso M, Muscatiello N. Drug-eluting beads versus conventional chemoembolization for the treatment of unresectable hepatocellular carcinoma: a metaanalysis. Dig Liver Dis. 2016;48:571-7.

124 Ikeda M, Inaba Y, Tanaka T, Sugawara S, Kodama Y, Aramaki T, et al. A prospective randomized controlled trial of selective transarterial chemoembolization using drug-eluting beads loaded with epirubicin versus selective conventional transarterial chemoembolization using epirubicin-lipiodol for hepatocellular carcinoma: the JIVROSG-1302 PRESIDENT study. J Clin Oncol. 2020;38(15 Suppl):4518 (suppl; abstr 4518).

125 Willett CG, Boucher Y, di Tomaso E, Duda DG, Munn LL, Tong RT, et al. Direct evidence that the VEGF-specific antibody bevacizumab has antivascular effects in human rectal cancer. Nat Med. 2004;10(2):145-7.

126 Yuan F, Chen Y, Dellian M, Safabakhsh N, Ferrara N, Jain RK. Time-dependent vascular regression and permeability changes in established human tumor xenografts induced by an anti-vascular endothelial growth factor/vascular permeability factor antibody. Proc Natl Acad Sci U S A. 1996; 93(25):14765-70.

127 Jain RK. Normalization of tumor vasculature: an emerging concept in antiangiogenic therapy. Science. 2005;307(5706):58-62.

128 Kano MR, Komuta Y, Iwata C, Oka M, Shirai YT, Morishita Y, et al. Comparison of the effects of the kinase inhibitors imatinib, sorafenib, and transforming growth factorbeta receptor inhibitor on extravasation of nanoparticles from neovasculature. Cancer Sci. 2009;100(1):173-80.

129 Jia ZZ, Jiang GM, Feng YL. Serum HIF-1alpha and VEGF levels pre- and post-TACE in patients with primary liver cancer. Chin Med Sci J. 2011;26(3):158-62.

130 Kudo M, Ueshima K, Ikeda M, Torimura T, Tanabe N, Aikata H, et al. Randomised, multicentre prospective trial of transarterial chemoembolisation (TACE) plus sorafenib as compared with TACE alone in patients with hepatocellular carcinoma: TACTICS trial. Gut. 2020 Aug;69(8):1492-501.
131 Kudo M, Imanaka K, Chida N, Nakachi K, Tak WY, Takayama T, et al. Phase III study of sorafenib after transarterial chemoembolisation in Japanese and Korean patients with unresectable hepatocellular carcinoma. Eur J Cancer. 2011;47(14):2117-27.

132 Lencioni R, Llovet JM, Han G, Tak WY, Yang J, Guglielmi A, et al. Sorafenib or placebo plus TACE with doxorubicin-eluting beads for intermediate stage HCC: the SPACE trial. J Hepatol. 2016;64(5):1090-8.

133 Meyer T, Fox R, Ma YT, Ross PJ, James MW, Sturgess R, et al. Sorafenib in combination with transarterial chemoembolisation in patients with unresectable hepatocellular carcinoma (TACE 2): a randomised placebocontrolled, double-blind, phase 3 trial. Lancet Gastroenterol Hepatol. 2017;2(8): 565-75.

134 Kudo M, Cheng A-L, Park J-W, Park JH, Liang $\mathrm{P}-\mathrm{C}$, Hidaka $\mathrm{H}$, et al. Orantinib versus placebo combined with transcatheter arterial chemoembolisation in patients with unresectable hepatocellular carcinoma (ORIENTAL): a randomised, double-blind, placebo-controlled, multicentre, phase 3 study. Lancet Gastroenterol Hepatol. 2018;3(1): $37-46$.

135 Kudo M, Han G, Finn RS, Poon RT, Blanc JF, Yan L, et al. Brivanib as adjuvant therapy to transarterial chemoembolization in patients with hepatocellular carcinoma: a randomized phase III trial. Hepatology. 2014; 60(5):1697-707.

136 Ogasawara S, Chiba T, Ooka Y, Kanogawa N, Motoyama T, Suzuki E, et al. Efficacy of sorafenib in intermediate-stage hepatocellular carcinoma patients refractory to transarterial chemoembolization. Oncology. 2014; 87(6):330-41.

137 Arizumi T, Ueshima K, Chishina H, Kono M, Takita M, Kitai S, et al. Validation of the criteria of transcatheter arterial chemoembolization failure or refractoriness in patients with advanced hepatocellular carcinoma proposed by the LCSGJ. Oncology. 2014; 87(Suppl 1):32-6.

138 Peck-Radosavljevic M, Raoul J, Lee HC, Kudo M, Nakajima K, Cheng AL. Practice patterns and outcomes of transarterial chemoembolization in patients with hepatocellular carcinoma who were ineligible and eligible for transarterial chemoembolization at inclusion. Global OPTIMIS exploratory analysis ILC 2019, FRI-494.

139 Kudo M, Finn RS, Qin S, Han K-H, Ikeda K, Piscaglia F, et al. Lenvatinib versus sorafenib in first-line treatment of patients with unresectable hepatocellular carcinoma: a randomised phase 3 non-inferiority trial. Lancet. 2018;391(10126):1163-73.
140 Yamashita T, Kudo M, Ikeda K, Izumi N, Tateishi R, Ikeda M, et al. REFLECT-a phase 3 trial comparing efficacy and safety of lenvatinib to sorafenib for the treatment of unresectable hepatocellular carcinoma: an analysis of Japanese subset. J Gastroenterol. 2020;55(1):113-22.

141 Ueshima K, Nishida N, Hagiwara S, Aoki T, Minami T, Chishina $\mathrm{H}$, et al. Impact of baseline ALBI grade on the outcomes of hepatocellular carcinoma patients treated with lenvatinib: a multicenter study. Cancers. 2019; 11:952.

142 Raoul JL, Gilabert M, Piana G. How to define transarterial chemoembolization failure or refractoriness: a European perspective. Liver Cancer. 2014;3(2):119-24.

143 Cheng AL, Amarapurkar D, Chao Y, Chen PJ, Geschwind JF, Goh KL, et al. Re-evaluating transarterial chemoembolization for the treatment of hepatocellular carcinoma: consensus recommendations and review by an International Expert Panel. Liver Int. 2014; 34(2):174-83.

144 Arizumi T, Ueshima K, Minami T, Kono M Chishina $\mathrm{H}$, Takita $\mathrm{M}$, et al. Effectiveness of Sorafenib in patients with transcatheter arterial chemoembolization (TACE) refractory and intermediate-stage hepatocellular carcinoma. Liver Cancer. 2015;4(4):253-62.

145 Peck-Radosavljevic M, Kudo M, Raoul J-L, Lee HC, Decaens T, Heo J, et al. Outcomes of patients (pts) with hepatocellular carcinoma (HCC) treated with transarterial chemoembolization (TACE): global OPTIMIS final analysis. J Clin Oncol. 2018;36(15 Suppl):4018 (suppl; abstr 4018).

146 Ikeda M, Mitsunaga S, Shimizu S, Ohno I, Takahashi H, Okuyama H, et al. Efficacy of sorafenib in patients with hepatocellular carcinoma refractory to transcatheter arterial chemoembolization. J Gastroenterol. 2014;49(5):932-40.

147 Kodama K, Kawaoka T, Aikata H, Uchikawa $S$, Inagaki $\mathrm{Y}$, Hatooka $\mathrm{M}$, et al. Comparison of clinical outcome of hepatic arterial infusion chemotherapy and sorafenib for advanced hepatocellular carcinoma according to macrovascular invasion and transcatheter arterial chemoembolization refractory status. J Gastroenterol Hepatol. 2018;33(10): 1780-6.

148 Kudo M. A new treatment option for intermediate-stage hepatocellular carcinoma with high tumor burden: initial lenvatinib therapy with subsequent selective TACE. Liver Cancer. 2019;8(5):299-311.

149 Kudo M, Ueshima K, Chan S, Minami T, Chishina $\mathrm{H}$, Aoki $\mathrm{T}$, et al. Lenvatinib as an initial treatment in patients with intermediate-stage hepatocellular carcinoma beyond up-to-seven criteria and Child-Pugh A liver function: a proof-of-concept study. Cancers. 2019 Jul 31;11(8):1084.
JSH Consensus Statements and

Recommendations on HCC

Liver Cancer 2021:10:181-223 
150 Kudo M, Han KH, Ye SL, Zhou J, Huang YH, Lin SM, et al. A changing paradigm for the treatment of intermediate-stage hepatocellular carcinoma: Asia-Pacific primary liver cancer expert consensus statements. Liver Cancer. 2020;9(3):245-60.

151 Kudo M. Heterogeneity and subclassification of Barcelona clinic liver cancer stage B. Liver Cancer. 2016;5(2):91-6.

152 Llovet JM, Real MI, Montaña X, Planas R, Coll S, Aponte J, et al. Arterial embolisation or chemoembolisation versus symptomatic treatment in patients with unresectable hepatocellular carcinoma: a randomised controlled trial. Lancet. 2002;359(9319):1734-9.

153 Llovet JM, Vilana R, Brú C, Bianchi L, Salmeron JM, Boix L, et al. Increased risk of tumor seeding after percutaneous radiofrequency ablation for single hepatocellular carcinoma. Hepatology. 2001;33(5):1124-9.

154 Bruix J, Reig M, Sherman M. Evidencebased diagnosis, staging, and treatment of patients with hepatocellular carcinoma. Gastroenterology. 2016;150(4):835-53.

155 Kudo M. Extremely high objective response rate of lenvatinib: its clinical relevance and changing the treatment paradigm in hepatocellular carcinoma. Liver Cancer. 2018;7(3): 215-24.

156 Kudo M, Arizumi T, Ueshima K, Sakurai T, Kitano M, Nishida N. Subclassification of BCLC B stage hepatocellular carcinoma and treatment strategies: proposal of modified Bolondi's subclassification (Kinki criteria). Dig Dis. 2015;33(6):751-8.

157 Mazzaferro V, Llovet JM, Miceli R, Bhoori S, Schiavo M, Mariani L, et al. Predicting survival after liver transplantation in patients with hepatocellular carcinoma beyond the Milan criteria: a retrospective, exploratory analysis. Lancet Oncol. 2009;10(1):35-43.

158 Arizumi T, Minami T, Chishina H, Kono M, Takita M, Yada N, et al. Time to transcatheter arterial chemoembolization refractoriness in patients with hepatocellular carcinoma in Kinki criteria stages B1 and B2. Dig Dis. 2017;35(6):589-97.

159 Yasui Y, Tsuchiya K, Kurosaki M, Takeguchi T, Takeguchi Y, Okada M, et al. Up-to-seven criteria as a useful predictor for tumor downstaging to within Milan criteria and Child-Pugh grade deterioration after initial conventional transarterial chemoembolization. Hepatol Res. 2018;48(6):442-50.

160 Kimura H, Ohkawa K, Miyazaki M, Sakakibara $\mathrm{M}$, Imanaka $\mathrm{K}$, Tamura $\mathrm{T}$, et al. Subclassification of patients with intermediatestage (Barcelona Clinic Liver Cancer stageB) hepatocellular carcinoma using the up-to-seven criteria and serum tumor markers. Hepatol Int. 2017;11(1):105-14.
161 Eso Y, Takai A, Takahashi K, Ueda Y, Taura $\mathrm{K}$, Marusawa $\mathrm{H}$, et al. Combination of Mac2 binding protein glycosylation isomer and up-to-seven criteria as a useful predictor for Child-Pugh grade deterioration after transarterial chemoembolization for hepatocellular carcinoma. Cancers. 2019;11(3):405.

162 Izumoto $\mathrm{H}$, Hiraoka A, Ishimaru Y, Murakami T, Kitahata S, Ueki H, et al. Validation of newly proposed time to transarterial chemoembolization progression in intermediate-stage hepatocellular carcinoma cases. Oncology. 2017;93(Suppl 1):120-6.

163 Hiraoka A, Kumada T, Kudo M, Hirooka M, Koizumi Y, Hiasa Y, et al. Hepatic function during repeated TACE procedures and prognosis after introducing sorafenib in $\mathrm{pa}$ tients with unresectable hepatocellular carcinoma: multicenter analysis. Dig Dis. 2017; 35(6):602-10.

164 Kanai T, Hirohashi S, Upton MP, Noguchi M, Kishi K, Makuuchi M, et al. Pathology of small hepatocellular carcinoma. A proposal for a new gross classification. Cancer. 1987; 60(4):810-9.

165 Yamashita Y, Matsukawa T, Arakawa A, Hatanaka Y, Urata J, Takahashi M. US-guided liver biopsy: predicting the effect of interventional treatment of hepatocellular carcinoma. Radiology. 1995;196(3):799-804.

166 Carmeliet P, Jain RK. Angiogenesis in cancer and other diseases. Nature. 2000; 407(6801):249-57.

167 Tohyama O, Matsui J, Kodama K, Hata-Sugi N, Kimura T, Okamoto K, et al. Antitumor activity of lenvatinib (e7080): an angiogenesis inhibitor that targets multiple receptor tyrosine kinases in preclinical human thyroid cancer models. J Thyroid Res. 2014; 2014:638747.

168 Kawamura Y, Kobayashi M, Shindoh J, Kobayashi Y, Kasuya K, Sano T, et al. (18)FFluorodeoxyglucose uptake in hepatocellular carcinoma as a useful predictor of an extremely rapid response to lenvatinib. Liver Cancer. 2020;9:84-92.

169 Kawamura Y, Kobayashi M, Shindo J, Kobayashi Y, Kasuya K, Sano T, et al. Pretreatment heterogeneous enhancement pattern of hepatocellular carcinoma may be a useful new predictor of early response to lenvatinib and overall prognosis. Liver Cancer. 2020 Jun;9:275-92. Epub ahead of print.

170 Kudo M, Ueshima K, Aikata H, Tamai T, Saito K, Ikeda K. Association between tumor response by mRECIST and overall survival in patients with poorly differentiated HCC in REFLECT study. 10th Asia-Pacific Primary Liver Cancer Expert

171 Ueshima K, Kudo M, Tanaka M, Kumada T, Chung H, Hagiwara S, et al. Phase I/II study of sorafenib in combination with hepatic arterial infusion chemotherapy using lowdose cisplatin and 5-fluorouracil. Liver Cancer. 2015;4(4):263-73.
172 Ikeda M, Shimizu S, Sato T, Morimoto M Kojima Y, Inaba Y, et al. Sorafenib plus hepatic arterial infusion chemotherapy with cisplatin versus sorafenib for advanced hepatocellular carcinoma: randomized phase II trial. Ann Oncol. 2016;27(11):2090-6.

173 Kudo M, Ueshima K, Yokosuka O, Ogasawara S, Obi S, Izumi N, et al. Sorafenib plus low-dose cisplatin and fluorouracil hepatic arterial infusion chemotherapy versus sorafenib alone in patients with advanced hepatocellular carcinoma (SILIUS): a randomised, open label, phase 3 trial. Lancet Gastroenterol Hepatol. 2018;3(6):424-32.

174 He M, Li Q, Zou R, Shen J, Fang W, Tan G, et al. Sorafenib plus hepatic arterial infusion of oxaliplatin, fluorouracil, and leucovorin vs sorafenib alone for hepatocellular carcinoma with portal vein invasion: a randomized clinical trial. JAMA Oncol. 2019;5(7): 953-60.

175 Ueshima K, Ogasawara S, Ikeda M, Yasui Y, Terashima T, Yamashita T, et al. Hepatic arterial infusion chemotherapy versus sorafenib in patients with advanced hepatocellular carcinoma. Liver Cancer. 2020 Sep; 9(5):583-95.

176 Terashima T, Yamashita T, Arai K, Sunagozaka H, Kitahara M, Nakagawa H, et al. Feasibility and efficacy of hepatic arterial infusion chemotherapy for advanced hepatocellular carcinoma after sorafenib. Hepatol Res. 2014;44(12):1179-85.

177 Llovet JM, Ricci S, Mazzaferro V, Hilgard P, Gane E, Blanc JF, et al. Sorafenib in advanced hepatocellular carcinoma. $\mathrm{N}$ Engl J Med. 2008;359(4):378-90.

178 Cheng AL, Kang YK, Chen Z, Tsao CJ, Qin S, Kim JS, et al. Efficacy and safety of sorafenib in patients in the Asia-Pacific region with advanced hepatocellular carcinoma: a phase III randomised, double-blind, placebo-controlled trial. Lancet Oncol. 2009;10(1):25-34.

179 Bruix J, Qin S, Merle P, Granito A, Huang YH, Bodoky G, et al. Regorafenib for patients with hepatocellular carcinoma who progressed on sorafenib treatment (RESORCE): a randomised, double-blind, placebo-controlled, phase 3 trial. Lancet. 2017;389(10064):56-66.

180 Zhu AX, Kang YK, Yen CJ, Finn RS, Galle $\mathrm{PR}$, Llovet JM, et al. Ramucirumab after sorafenib in patients with advanced hepatocellular carcinoma and increased alpha-fetoprotein concentrations (REACH-2): a randomised, double-blind, placebo-controlled, phase 3 trial. Lancet Oncol. 2019;20:282-96.

181 Abou-Alfa GK, Meyer T, Cheng AL, ElKhoueiry AB, Rimassa L, Ryoo BY, et al. Cabozantinib in patients with advanced and progressing hepatocellular carcinoma. N Engl J Med. 2018;379(1):54-63. 
182 Kudo M, Tsuchiya K, Kato N, Hagihara A, Numata K, Aikata $\mathrm{H}$, et al. Cabozantinib in Japanese patients with advanced hepatocellular carcinoma: a phase 2 multicenter study. J Gastroenterol. 2021;56(2):181-90.

183 El-Khoueiry AB, Sangro B, Yau T, Crocenzi TS, Kudo M, Hsu C, et al. Nivolumab in patients with advanced hepatocellular carcinoma (CheckMate 040): an open-label, noncomparative, phase $1 / 2$ dose escalation and expansion trial. Lancet. 2017;389(10088): 2492-502.

184 Zhu AX, Finn RS, Edeline J, Cattan S, Ogasawara S, Palmer D, et al. Pembrolizumab in patients with advanced hepatocellular carcinoma previously treated with sorafenib (KEYNOTE-224): a non-randomised, openlabel phase 2 trial. Lancet Oncol. 2018;19(7): 940-52.

185 Finn RS, Ryoo B-Y, Merle P, Kudo M, Bouattour M, Lim HY, et al. Pembrolizumab as second-line therapy in patients with advanced hepatocellular carcinoma in KEYNOTE-240: a randomized, double-blind, phase III trial. J Clin Oncol. 2020;38(3):193202.

186 Yau T, Park JW, Finn RS, Cheng AL, Mathurin P, Edeline J, et al. CheckMate 459: a randomized, multi-center phase III study of nivolumab vs sorafenib as first-line treatment in patients with advanced hepatocellular carcinoma. Ann Oncol. 2019;30(Suppl 5):v874-5.

187 Finn RS, Qin S, Ikeda M, Galle PR, Ducreux M, Kim T-Y, et al. Atezolizumab plus bevacizumab in unresectable hepatocellular carcinoma. N Engl J Med. 2020;382(20):1894905.

188 Kudo M. A paradigm change in the treatment strategy for hepatocellular carcinoma. Liver Cancer. 2020;9(4):367-77.
189 Kudo M. A new era in systemic therapy for hepatocellular carcinoma: atezolizumab plus bevacizumab combination therapy. Liver Cancer. 2020;9(2):119-37.

190 Finn RS, Ikeda M, Zhu AX, Sung MW, Baron AD, Kudo M, et al. Phase Ib study of lenvatinib plus pembrolizumab in patients with unresectable hepatocellular carcinoma. J Clin Oncol. 2020 Sep 10;38(26):2960-70.

191 Kelley RK, Kudo M, Harris W, Ikeda M, Okusaka T, Kang YK, et al. The novel regimen of tremelimumab in combination with durvalumab provides a favorable safety profile and clinical activity for patients with advanced hepatocellular carcinoma. ILCA. 2020. (Virtual Conference).

192 Yau T, Kang YK, Kim TY, El-Khoueiry AB, Santoro A, Sangro B, et al. Nivolumab plus ipilimumab in advanced hepatocellular carcinoma previously treated with sorafenib (CheckMate 040): a randomized clinical trial. JAMA Oncol. 2020 Oct 1;6(11):e204564.

193 Kudo M. Immuno-oncology therapy for hepatocellular carcinoma: current status and ongoing trials. Liver Cancer. 2019;8(4):22138.

194 Hiraoka A, Kumada T, Kariyama K, Takaguchi K, Atsukawa M, Itobayashi E, et al. Clinical features of lenvatinib for unresectable hepatocellular carcinoma in real-world conditions: multicenter analysis. Cancer Med. 2019;8(1):137-46.

195 The Japanese Liver Transplantation Society. Liver transplantation in Japan-Registry by the Japanese Liver Transplantation Society. 2019;54:81-96.
196 Nouso K, Miyahara K, Uchida D, Kuwaki K, Izumi $\mathrm{N}$, Omata $\mathrm{M}$, et al. Effect of hepatic arterial infusion chemotherapy of 5-fluorouracil and cisplatin for advanced hepatocellular carcinoma in the Nationwide Survey of Primary Liver Cancer in Japan. Br J Cancer. 2013;109(7):1904-7.

197 Bruix J, Raoul JL, Sherman M, Mazzaferro V, Bolondi L, Craxi A, et al. Efficacy and safety of sorafenib in patients with advanced hepatocellular carcinoma: subanalyses of a phase III trial. J Hepatol. 2012;57(4):821-9.

198 Kokudo T, Hasegawa K, Matsuyama Y, Takayama T, Izumi N, Kadoya M, et al. Survival benefit of liver resection for hepatocellular carcinoma associated with portal vein invasion. J Hepatol. 2016;65(5):938-43.

199 Kokudo T, Hasegawa K, Matsuyama Y, Takayama T, Izumi N, Kadoya M, et al. Liver resection for hepatocellular carcinoma associated with hepatic vein invasion: a Japanese nationwide survey. Hepatology. 2017; 66(2):510-7.

200 Kudo M. Management of hepatocellular carcinoma in Japan as a world-leading model. Liver Cancer. 2018;7:134-47.

201 Kudo M. Japan's successful model of nationwide hepatocellular carcinoma surveillance highlighting the urgent need for global surveillance. Liver Cancer. 2012;1(3-4):141-3.

202 Kudo M, Izumi N, Sakamoto M, Matsuyama Y, Ichida T, Nakashima O, et al. Survival analysis over 28 years of 173,378 patients with hepatocellular carcinoma in Japan. Liver Cancer. 2016;5(3):190-7.

203 Kudo M, Lencioni R, Marrero JA, Venook AP, Bronowicki JP, Chen XP, et al. Regional differences in sorafenib-treated patients with hepatocellular carcinoma: GIDEON observational study. Liver Int. 2016;36(8): 1196-205.
JSH Consensus Statements and Recommendations on HCC
Liver Cancer 2021:10:181-223 